\title{
A Frequency-dependent Multiconductor Transmission Line Model with Collocated Voltage and Current Propagation
}

\author{
by \\ Arash Tavighi \\ M.Sc. University of Tehran, 2008
}

A THESIS SUBMITTED IN PARTIAL FULFILLMENT

OF THE REQUIREMENTS FOR THE DEGREE OF

Doctor of Philosophy

in

THE FACULTY OF GRADUATE AND POSTDOCTORAL STUDIES

(Electrical and Computer Engineering)

The University of British Columbia

(Vancouver)

March 2017

(C) Arash Tavighi, 2017 


\section{Abstract}

This research contributes to developing a time domain and a frequency domain formulations to solve electromagnetic transients in power system with multiconductor overhead transmission lines.

The time domain solution introduces a frequency dependent transmission line model "FDLM". For the development of the FDLM a fundamental constraint is added to the classical line equations to maintain the symmetry between electric and magnetic fields. As a result, voltage waves and current waves travel together and the characteristic impedance remains uniform along the line. With this premise, a constant real transformation matrix can be obtained to diagonalize the line functions with high accuracy. This feature can greatly facilitate the line modelling as opposed to the existing line models which require complex frequency dependent transformation matrices for their diagonalization. The use of a single constant real transformation matrix for the voltage and current waves which is exact over the frequency range enables FDLM to provide higher accuracy and numerical efficiency than the existing line models while it complies with the physical system.

The accuracy of the FDLM is assessed through comparisons with a newly developed Discrete Time Fourier Series frequency domain solution. This methodology is based on the correct specification of the time window and frequency window widths. Guidelines are provided for this set up which avoids the typical Gibbs and aliasing errors related to the classical frequency domain solutions. The proposed frequency domain solution is simpler to implement than the most commonly used numerical Laplace transform solution while it does not require further considerations to use damping factors or windowing functions. 


\section{Preface}

Based on the research presented in this dissertation, several papers have been published in and/or submitted to conference proceedings and journal articles. In all of the publications, I have implemented the models, developed the case studies, conducted simulations, analyzed results, prepared the initial manuscript (except for the last paper). My supervisor Prof. José R. Martí has provided me instructive comments and corrections throughout the process of conducting research studies, preparing and editing manuscripts.

Dr. J. A. Gutierrez-Robles from the University of Guadalajara provided the Laplace method for our benchmarking purposes. Verónica A. Galván, a visiting scholar from Cinvestav, extended the Laplace method for various case studies. Professor Hermann W. Dommel provided us many instructive discussion sessions on transients studies and transmission line modelling.

The following describes published and submitted papers.

- Chapter 2 was presented at the IPST 2015 conference. A. Tavighi, J. R. Martí, and J. A. Gutierrez-Robles, "Comparison of the fdLine and ULM Frequency Dependent EMTP Line Models with a Reference Laplace Solution", International Conference on Power Systems Transients (IPST2015), Cavtat, Croatia, June 15-18, 2015.

- Part of Chapter 3 submitted as a journal article. A. Tavighi, V. A. Galván, J. R. Martí, and J. A. Gutierrez-Robles "Validation and Usage Guidelines for Discrete Time Fourier Series to Solve Electromagnetic Transients in Power Systems".

- Part of Chapter 3 submitted as a conference paper. A. Tavighi, J. R. Martí, V. A. Galván, J. A. Gutierrez-Robles, and H. W. Dommel, “A Discrete Time Fourier Series Formulation to Simulate Electromagnetic Transients for Multi-conductor 
Transmission Lines".

- Chapter 4 submitted as a journal article. J. R. Martí, and A. Tavighi, "Frequency Dependent Multiconductor Transmission Line Model with Collocated Voltage and Current Propagation". 


\section{Table of Contents}

Abstract .......................... ii

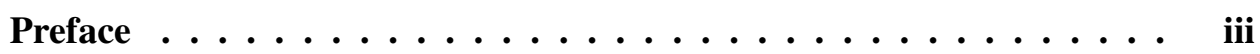

Table of Contents $\ldots \ldots \ldots \ldots \ldots \ldots$

List of Tables $\ldots \ldots \ldots \ldots \ldots \ldots \ldots$ viii

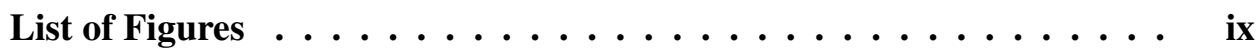

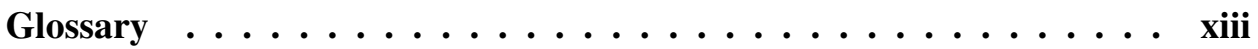

Acknowledgments ..................... X

Dedication $\ldots \ldots \ldots \ldots \ldots \ldots \ldots \ldots \ldots \ldots \ldots \ldots \ldots \ldots \ldots$

1 Introduction ........................ 1

1.1 Motivation . . . . . . . . . . . . . . . 1

1.2 History of Line Parameters Calculation . . . . . . . . . . 3

1.3 History of Frequency Domain Solutions . . . . . . . . . . . 6

1.4 History of Time Domain Line Models . . . . . . . . . . . . . 7

1.5 Research Objectives and Anticipated Impacts . . . . . . . . . . 11

1.5.1 Validation of Frequency-Dependent Line (FDLINE) Under Asymmetrical Line Configurations . . . . . . . . . . 12

1.5.2 Development of an Accurate Frequency Domain Solution 12 
1.5.3 Revisions to the Classical Multiconductor Transmission Line (MTL) Equations and Development of a Frequency Dependent Electro-Magnetic Transients Program (EMTP) Line Model

Based on the Revised Equations . . . . . . . . . . 12

2 Accuracy of FDLINE Under Asymmetrical Line Configurations . . . 14

2.1 Classical MTL Equations . . . . . . . . . . . . . 15

2.1.1 Per Unit Length $\mathbf{Z}$ and $\mathbf{Y}$ Calculation $\ldots \ldots \ldots \ldots$

2.1.2 Diagonalization of the MTL Equations . . . . . . . . . 19

2.2 Time Domain and Frequency Domain Solutions to the MTL Equations 21

2.2.1 FDLINE Solution to the MTL Equations . . . . . . . . 21

2.2.2 Universal Line Model (ULM) Solution to the MTL Equations 24

2.2.3 Numerical Laplace Transform (NLT) Solution to the MTL Equations . . . . . . . . . . . . . . 25

2.3 Case Studies and Simulation Conditions . . . . . . . . . . . 28

2.4 Comparison of Simulation Results . . . . . . . . . . . . . 33

2.4.1 Single-Phase Line . . . . . . . . . . . . . 33

2.4.2 Three-Phase Single-Circuit Lines . . . . . . . . . . 34

2.4.3 Three-Phase Double-Circuit Line in the Same Tower . . . 35

2.4.4 Three-Phase Double-Circuit Line in Separate Towers . . . 37

2.4.5 Steady-state Solutions . . . . . . . . . . . . 38

2.5 General Observations . . . . . . . . . . . . . . . . 38

3 Discrete Time Fourier Series Algorithm . . . . . . . . . . . . 40

3.1 Relationship Between Discrete Time Domain and Discrete Frequency Domain . . . . . . . . . . . . . . .

3.2 Power System Transient Solution Using the Discrete Time Fourier Series (DTFS) . . . . . . . . . . . . . . . . . . 43

3.2.1 Defining the Time Window Width $\left(T_{c}\right) \ldots \ldots \ldots$

3.2.2 Defining the Frequency Window Width $\left(f_{c}\right) \ldots \ldots$

3.2.3 System Solution Using the DTFS . . . . . . . . . . . 46

3.3 Guidelines to Specify $T_{c}$ and $f_{c} \ldots \ldots \ldots \ldots$

3.3.1 Calculation of the Time Window Width $\left(T_{c}\right) \ldots \ldots 47$ 
3.3.2 Calculation of the Frequency Window Width $\left(f_{c}\right) \ldots \ldots$

3.4 Case Studies . . . . . . . . . . . . . . . . . . . . . 49

3.4.1 Single-phase Circuits . . . . . . . . . . . . 50

3.4 .2 MTL-Circuits . . . . . . . . . . . . . . 51

3.5 Simulation Results . . . . . . . . . . . . . . . . 54

3.5.1 RL Circuit Simulation . . . . . . . . . . . . . 57

3.5.2 Nominal- $\pi$ Circuit Simulation . . . . . . . . . . . 62

3.5.3 Exact- $\pi$ Circuit Simulation . . . . . . . . . . . . 62

3.5.4 MTL-Circuits Simulation . . . . . . . . . . . . . 64

3.6 Sensitivity of the DTFS to the Width of the $t_{\text {set }} \ldots \ldots \ldots$

3.7 General Observations . . . . . . . . . . . . . . . 72

3.8 Sensitivity of FDLINE to the Frequency of Approximation for the Transformation Matrices . . . . . . . . . . . . . 72

4 Revised Multiconductor Transmission Line Equations, Proposed FrequencyDependent Line Model . . . . . . . . . . . . . . . . . 78

4.1 Inconsistencies in the Classical MTL Equations . . . . . . . . 79

4.2 Collocation of Current and Voltage Waves . . . . . . . . . . 80

4.3 Effect of the Earth Return in the Matrix of Impedances . . . . . . 81

4.4 Validity of the Collocation Condition $\mathbf{Z Y}=\mathbf{Y Z} \ldots \ldots$. . . . . 84

4.5 Diagonalization of the Revised Multiconductor Transmission Line (RMTL) Equations . . . . . . . . . . . . . . . . . 85

4.6 Transformation Matrix $\mathbf{T}$ to Diagonalize $\mathbf{Z}$ and $\mathbf{Y} \ldots \ldots$

4.7 Transformation Matrix $\mathbf{T}$ to Diagonalize $\mathbf{Z Y}$ and $\mathbf{Y Z} \ldots . . . .89$

4.8 Proposed Frequency-Dependent Line Model . . . . . . . . . . . . 91

4.9 Numerical Results: Transient Simulations . . . . . . . . . . . . . 94

4.10 General Observations . . . . . . . . . . . . . . . . . . . 98

4.11 Further Investigations on the Wave Collocation Condition . . . . . 100

5 Summary of Contributions and Future Works $\ldots \ldots 7$

5.1 Conclusions and Contributions . . . . . . . . . . . . . 107

5.2 Future Works . . . . . . . . . . . . . . . . . . 109

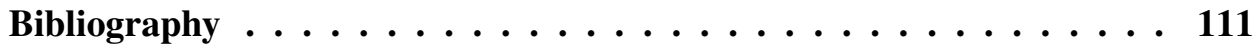




\section{List of Tables}

Table 2.1 Comparison of steady state values of different solutions. . . . . 39

Table 3.1 Analytical formulas for the RL and nominal- $\pi$ circuits. . . . . 52

Table 3.2 Modal lumped elements of the nominal- $\pi$ circuit for the double circuit vertical line of Fig. 3.5 calculated at $10^{-4} \mathrm{~Hz}$. . . . . . 52

Table 3.3 Time-frequency parameters for the DTFS. . . . . . . . . . 56

Table 4.1 Errors in the diagonalization of the matrix of ones $[\mathbf{1}]$ and $\mathbf{C}_{e x t}$ for $\mathbf{T}$ calculated at $100 \mathrm{~Hz}$. . . . . . . . . . . . . 89

Table 4.2 Real part of the eigenvalues of $\mathbf{Z Y}$ calculated using the proposed single $\mathbf{T}$ versus exact diagonalization (all values are negative). . . . . . . . . . . . . . . .

Table 4.3 Imaginary part of the eigenvalues of $\mathbf{Z Y}$ calculated using the proposed single $\mathbf{T}$ versus exact diagonalization (all values are negative). . . . . . . . . . . . . . .

Table 4.4 Maximum errors for voltages and currents for FDLINE, ULM, and Frequency-Dependent Line Model (FDLM) with respect to their reference DTFS solution. . . . . . . . . . . . . . . 96

Table 4.5 Comparison of operations count for FDLINE, ULM, and FDLM line models in the simulation of unbalanced faults test of Fig. 3.6. 97 


\section{List of Figures}

Figure 2.1 Transmission line segment $\Delta x$ (only one conductor with respect to ground is shown). . . . . . . . . . . .

Figure 2.2 Conductors above non-ideal ground using the method of images and complex penetration depth. . . . . . . . . 17

Figure 2.3 FDLINE modal equivalent circuit. . . . . . . . . . . . 22

Figure 2.4 Coupled exact- $\pi$ model. Frequency domain equivalent-circuit for multiconductor transmission line. . . . . . . . . 27

Figure 2.5 Single-phase transmission line (open and shorted). . . . . . . 29

Figure 2.6 Three-phase single-circuit horizontal transmission line. . . . . 29

Figure 2.7 Three-phase single-circuit vertical transmission line. . . . . . 30

Figure 2.8 Three-phase single-circuit delta transmission line. . . . . . . . 30

Figure 2.9 Three-phase double-circuit one-tower delta transmission line. 31

Figure 2.10 Three-phase double-circuit two-tower horizontal transmission line. . . . . . . . . . . . . . . . . . 31

Figure 2.11 One-line diagram of the equivalent circuit for the tests. a) Single-circuit, b) Double-circuit. . . . . . . . . . . . 32

Figure 2.12 Simulation results for the single-phase line of Fig. 2.5 . . . . 33

Figure 2.13 Simulation results for the horizontal line of Fig. 2.6. . . . . . 34

Figure 2.14 Simulation results for the vertical line of Fig. 2.7 . . . . . . 35

Figure 2.15 Simulation results for the delta line of Fig. 2.8 . . . . . . 36

Figure 2.16 Simulation results for the double circuit delta line of Fig. 2.9. 37

Figure 2.17 Simulation results for the two parallel horizontal line of Fig.

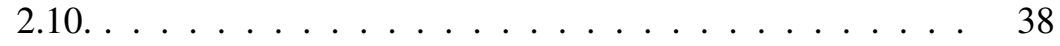


Figure 3.1 Correspondence of time domain and frequency domain for a discrete signal. a) $N_{s}=$ even. b) $N_{s}=$ odd. . . . . . . . . . . .

Figure 3.2 Time window selection. a) System impulse response $h[n]$, b) Current source $i[n]$ (Dashed line: unit step with discontinuity, Solid line: unit step with the averaging of points at the discontinuity $\ldots \ldots \ldots \ldots \ldots \ldots$. . . . . . . . . . 44

Figure $3.3 \quad$ Example single-phase overhead transmission line. . . . . . . 50

Figure 3.4 Single-phase line models. a) exact- $\pi$, b) nominal- $\pi$, c) RL. . . 50

Figure 3.5 Double circuit vertical transmission line. . . . . . . . . . . 53

Figure 3.6 System diagram for the unbalanced faults test. . . . . . . . . . 54

Figure 3.7 System diagram for the induced voltage test. . . . . . . . . . 54

Figure 3.8 System diagram for the step response test. . . . . . . . . . . . 55

Figure $3.9 i_{s c}$ for the RL circuit energized with a step voltage. . . . . . . 57

Figure 3.10 Close up of $i_{s c}$ for the RL circuit energized with the step voltage for $t_{s i m}=0.5 \mathrm{~s} . \ldots \ldots \ldots \ldots \ldots$

Figure 3.11 Effect of different windowing functions to reduce the oscillations of the NLT solution in Fig. 3.10. . . . . . . . . 59

Figure $3.12 v_{L}$ for the RL circuit energized with the step voltage. . . . . .

Figure 3.13 Test of NLT with odd sampling in the simulation of $v_{L}$ for the RL circuit . . . . . . . . . . . . . . . . . . . 60

Figure 3.14 The effect of filtering on the accuracy of NLT with odd sampling. Simulation of $v_{L}$ for the RL circuit. . . . . . . . 61

Figure $3.15 i_{s c}$ for the nominal- $\pi$ circuit energized with the step voltage. . 62

Figure 3.16 Details of Fig. 3.15 illustrating the effect of the first-last point

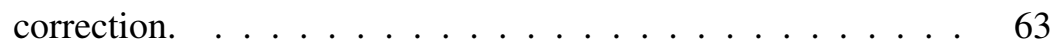

Figure $3.17 i_{s c}$ for the exact- $\pi$ circuit energized with the step voltage. . . . 63

Figure $3.18 i_{s c}$ for the exact- $\pi$ circuit energized with the cosine voltage. . 64

Figure 3.19 Error in the DTFS due to incorrect selection of the time window. 65

Figure $3.20 v_{3}$ in the unbalanced faults test of Fig. 3.6. . . . . . . . . 66

Figure $3.21 i_{6}$ in the unbalanced faults test of Fig. 3.6 . . . . . . . . 67

Figure $3.22 v_{6}$ and $i_{2}$ in the induced voltage test of Fig. 3.7. . . . . . . 67

Figure $3.23 v_{1}$ in the step response test of Fig. 3.8 . . . . . . . 68 
Figure 3.24 The real part of $\mathbf{T}_{I}(\omega)$ for the double circuit vertical line of Fig. 3.5. a) with modal switching. b) corrected for modal switching. . . . . . . . . . . . . .

Figure $3.25 v_{4}$ in the step response test of Fig. 3.8 illustrating the effect of modal switching problem. . . . . . . . . . .

Figure 3.26 Sensitivity of the DTFS method to the width of the $t_{\text {set }}$ for different tests. a) Relative error, b) Computational time. . . . . . 71

Figure 3.27 Sensitivity of FDLINE to the frequency of approximation . . . 74

Figure 3.28 Close up of $v_{3}$ and $i_{6}$ in the unbalanced faults test of Fig. 3.6. . 75

Figure 3.29 Close up of $i_{2}$ and $v_{6}$ in the induced voltage test of Fig. 3.7. . 76

Figure $3.30 v_{1}$ in the step response test of Fig. 3.8 . . . . . . . . . 77

Figure 4.1 Test circuit to determine the self and mutual impedances in the series impedance matrix Z . . . . . . . . . . . . .

Figure 4.2 Comparison of $\mathbf{R}_{e}$ in MTL and RMTL models for the double circuit vertical line of Fig. 3.5 . . . . . . . . . . . . . 84

Figure 4.3 Validity of the collocation condition. If $\mathbf{Z Y}=\mathbf{Y Z}$, the product $\mathbf{Z Y}(\mathbf{Y Z})^{-1}$ should be one for the diagonals and zero for the off-diagonals. . . . . . . . . . . . . . . . 85

Figure 4.4 RMTL formulation. T matrix to diagonalize $\mathbf{L}_{\text {loop }}$ for the line of Fig. 3.5. The same matrix $\mathbf{T}$ diagonalizes $\mathbf{Z Y}$ and $\mathbf{Y Z}$. . . .

Figure 4.5 MTL formulation. Real and imaginary part of complex $\mathbf{T}_{V}$ and $\mathbf{T}_{I}$ matrices to diagonalize $\mathbf{Z Y}$ and $\mathbf{Y Z}$ for the line of Fig. 3.5.

Figure 4.6 Diagonalization of $\mathbf{T}^{-1} \mathbf{Z Y T}$ and $\mathbf{T}^{-1} \mathbf{Y Z T}$. The plots show the absolute value of the off-diagonal elements. . . . . . . . . .

Figure 4.7 FDLM equivalent circuit in the frequency domain. Quantities can be matrices in the phase domain or scalar quantities in the modal domain. . . . . . . . . . . . . .

Figure 4.8 Comparison of MTL (FDLINE, ULM) and RMTL (FDLM) line models for the unbalanced fault test of Fig. 3.6. . . . . . . . 95

Figure 4.9 Details of the first four peaks of $v_{3}$ in Fig. 4.8a. . . . . . . . 96 
Figure 4.10 Errors for the voltage $v_{3}$ for FDLINE and ULM versus DTFS for the MTL equations and for FDLM versus DTFS for the RMTL equations. . . . . . . . . . . . . .

Figure $4.11 v_{1}$ in the step response test of Fig. 3.8. Comparison of RMTL models: DTFS versus FDLM (for FDLM T calculated at different frequencies). . . . . . . . . . . . . .

Figure 4.12 Earth transversal losses given by the complex penetration depth formula for the double circuit vertical line of Fig. 3.5 . . . . . 101

Figure $4.13 \mathbf{L}_{\text {loop }}$ and $\mathbf{C}_{\text {loop }}$ for the line of Fig. 3.5 . . . . . . . . 102

Figure 4.14 Validity of the collocation condition. If $\mathbf{Z Y}=\mathbf{Y Z}$, the product $\mathbf{Z Y}(\mathbf{Y Z})^{-1}$ should be one for the diagonals and zero for the off-diagonals. . . . . . . . . . . . . . 103

Figure 4.15 The effect of skin effect in capacitances on the product $\mathbf{L C}$ for the double circuit vertical line of Fig. 3.5 . . . . . . . . . 104

Figure 4.16 Comparison of MTL and RMTL models for two conditions: a) no skin in C, b) with skin in C. $v_{3}$ in the test of Fig. 3.6. . . . 105

Figure 4.17 Comparison of MTL with $\mathbf{C}_{\text {ext }}$ versus RMTL with $\mathbf{C}_{\text {loop }} . v_{3}$ in the test of Fig. 3.6. . . . . . . . . . . . . 105 


\title{
Glossary
}

\author{
ACSR Aluminum Conductor Steel Reinforced \\ BAF Bode's Asymptotic Fitting \\ BPA Bonneville Power Administration \\ DTFS Discrete Time Fourier Series \\ EMTP Electro-Magnetic Transients Program \\ FDLINE Frequency-Dependent Line \\ FDLM Frequency-Dependent Line Model \\ FEM Finite Element Method \\ FFT Fast Fourier Transform \\ IDTFS Inverse Discrete Time Fourier Transform \\ IFFT Inverse Fast Fourier Transform \\ MFT Modified Fourier Transform \\ MTL Multiconductor Transmission Line \\ NLT Numerical Laplace Transform \\ NSERC Natural Science and Engineering Research Council \\ PPC Points Per Cycle
}


RMTL Revised Multiconductor Transmission Line

TEM Transverse Electric Magnetic

UBC University of British Columbia

ULM Universal Line Model

VF Vector-Fitting 


\section{Acknowledgments}

Foremost, I would like to express my sincere gratitude to my supervisor Prof. José R. Martí for his continuous support, patience, great inspiration, immense knowledge and insights, and enthusiasm that he provided me throughout my studies. I appreciate all his contribution of time and ideas to make my doctoral experience productive and stimulating. I am also grateful to him for the knowledge that I acquired from his "Network Analysis and Simulation" course, and for the wonderful teaching experience that I gained while assisting him for the "Power System Analysis 1 and 2" courses. The financial support for this research was made possible by the Natural Science and Engineering Research Council (NSERC) of Canada under a grant led by Prof. José R. Martí as a principal investigator.

I would like to express my appreciation to Dr. J. A. Gutierrez-Robles and Verónica A. Galván for their valuable discussions on frequency domain solutions during their visit at the University of British Columbia (UBC).

My sincere thanks also go to Prof. Hermann W. Dommel for his valuable suggestions and knowledge contributions during the development of this research, and to Prof. Juri Jatskevich for his constructive comments on the presentation of contents of this dissertation and his magnificent lectures on "Dynamic Modelling of Electrical Machines and Controls" course.

Finally, I would like to thank my colleagues graduate students in Electric Power and Energy Systems research group at UBC: William Wang, Pouya Zadkhast, Mohammad Ghasemi, Aurash Alimardani, Francis Therrien, Ehssan Ghahremani, and Andrea Martí for many stimulating discussions and all the memorable moments we have had in the past five years at UBC. 


\section{Dedication}

To Maria and My Beloved Parents 


\section{Chapter 1}

\section{Introduction}

\subsection{Motivation}

Overhead transmission lines and underground cables transfer electric power over many kilometers from the generation sector to the customers at the distribution levels. These links are usually affected by over-voltages and over-currents caused by wide variety of transients such as switching operations, short circuits, lightning discharges, and faulty insulation. If these stresses imposed to the transmission lines are beyond a sustainable limit, these links can be severely damaged. To restrain the amplitude and duration of these undesirable phenomena, protective devices such as surge arresters and fault detection relays should be employed, and their location and setting should be tactfully determined. The success of the protective strategies to disconnect the lines during the transients depends on the accurate prediction of the system. This can be done through simulations when accurate transmission line models are available.

Two main approaches can be used to solve electric transients in frequencydependent transmission lines: Time domain and frequency domain solutions.

The frequency domain solution describes the transmission line one frequency at the time over a frequency range, and transforms the frequency response into a time response using time-frequency transformations such as the Fourier Transform. These models can analytically describe the frequency dependence of the circuit parameters, and unlike the time domain solutions they make no approximations 
when bridging the time response to the frequency response. Therefore, frequency domain solutions are valuable tools to assess the accuracy of the corresponding time-domain models. However, frequency domain solutions have difficulty to represent directly the opening and closing of circuit breakers at specific times and model nonlinear elements. Simulation of switching operations in frequency domain solutions requires going back and forth between frequency and time domains for each change of switch position, thus, making the process quite long and difficult. In terms of the computational costs, frequency domain solutions are more expensive than the time domain solutions as they require a one-to-one correspondence between frequency and time domains. This restricts their applications to be used as general purpose solvers.

Time domain methods are more flexible for circuits with switching operations, non-linear and time-varying elements, and to perform real-time analysis. Among the time-domain methods, the Electro-Magnetic Transients Program (EMTP) [29] [30] [28] is the most widely used tool. In direct time domain simulations, every element of an electrical network is described by a set of differential equations. In order to obtain the time-domain models of these elements, the corresponding differential equations are solved (integrated) between discrete time intervals (e.g. trapezoidal rule in the EMTP. The main handicap of time-domain methods is modelling frequency dependent components. Nonetheless, very good frequency dependent transmission line and underground cable models exist in the EMTP, as for example, Frequency-Dependent Line (FDLINE) [69], fqLine [71], and Universal Line Model (ULM) [74]. These models approximate the frequency dependence of the line or cable wave functions with rational function approximations (using, for example, Bode's Asymptotic Fitting (BAF) [1] and Vector-Fitting (VF) [55]). The fitted functions can then be transferred into the time domain to incorporate these models into the time domain solution. This conversion has to be done numerically, as a result, selection of the time step and numerical stability can affect the accuracy of the modelling [68]. Besides other factors such as consideration of the transformation matrices to diagonalize the line functions, the accuracy of the curve-fitting algorithms can also determine the accuracy of these line models.

The problem of frequency dependent transmission line modelling has been the subject of many researches for many years. Despite the significant progress made 
in this area, there is still room to further advance the modelling by taking into account physical assumptions.

In this section, the advances in the calculation of line parameters used in the classical Multiconductor Transmission Line (MTL) equations are briefly reviewed. The history of the most well-known frequency domain solutions and time domain line models are presented along with their pros and cons in preparation of proposing the Revised Multiconductor Transmission Line (RMTL) equations as the main objective of this research.

\subsection{History of Line Parameters Calculation}

\section{Traditional Approach}

The MTL equations belong to the class of formulations where the skin effect is only considered in the series impedance; while the shunt admittance is calculated assuming electrostatic position for the charges.

Sommerfeld in 1909 proposed a pioneering solution to the problem of "electromagnetic wave propagation along current-carrying conductors above a finitely conducting plane" [112]. In 1926, Carson proposed Quasi-Transverse Electric Magnetic (TEM) fields approximations of the Maxwell equations to solve this problem for an overhead transmission line [13]; whereas Pollaczek developed similar formulas applicable not only to overhead transmission lines but also to the underground cables and to combinations of both [98] [97]. Although Carsons approximations are relatively simple, they are valid for limited range of frequencies only that the capacitive displacement currents in the ground can be neglected. To validate Carsons equations for high frequencies, Semlyen presented an analytical continuation to the Carson's integral [106], and Hofmann derived an asymptotic series expansion for the calculation of the self and mutual line impedances [58].

There have been several attempts to approximate Carsons integral aiming to lessen the computational time of the simulation. First, Dubanton in 1969 suggested a simple and sufficiently accurate expressions for line impedances for the whole range of frequencies with intuitive insight [31]. Dubanton's formula was analytically proven by Gary in 1976 [41] and Deri in 1981 [26] introduced as the "complex ground return plane approach" with further improvement in [105] [110] 
[4]. Other authors presented approximations to Carson's formula in a form of double logarithmic [96] with further enhancement to obtain a simpler and more accurate closed-form formula in [87], and a more recent formulation based on the variable changes integral in [61]. Numerical evaluation of the Carson's integral were presented in [100] [86] [64] to assess the accuracy of approximation formulas.

\section{Stratified Earth}

Carson's formula was developed for the homogeneous infinite earth. In practice, however, the earth is not homogeneous, and its resistivity varies along the depth of the earth layer. Even if considered as an equivalent homogeneous earth, the resultant earth resistivity may be frequency dependent.

In 1949, Sunde [113] formulated the series impedance to include a two-layer earth only for overhead lines or cables above the earth. In his solution, however, the boundary conditions are not sufficiently general, and the propagation of current along a line is neglected [83]. The idea of Carson's integral extension for a twolayer earth was followed in [59] [78]. In 1973, Nakagawa proposed a more rigorous solution for a three layers earth case [83], with a sensitivity analysis for arbitrary earth resistivities, pemeabilities and permittivities [6], and with further extension to a general multi-layer case with continuously variable (exponential or linear) earth resistivity [82]. Nakagawa found out that the homogeneity assumption is only permissible at very high frequencies. Indeed, at low frequencies, a stratified earth causes significant differences in the earth impedances and the resultant wave deformations from the homogeneous case. He also concluded that the displacement currents mostly affect the earth-return impedances at frequencies beyond $1 \mathrm{MHz}$ and under the conditions that the earth resistivity is high and the height of the conductors above the ground is low [83].

Further studies in the modelling of the stratified earth include a direct numerical integration using Finite Element Method (FEM) [91] and closed-form formulas for a two layers earth [8] [119] with further generalization for a multi-layer earth [121] [120].

\section{Correction on Admittance}

As discussed, the MTL equations are based on the TEM assumption under which the ground return correction is only applied to the series impedance; while the shunt 
admittance is considered only of the physical geometry. Under this condition, it is assumed that the conductor and the earth surface have the same potential [40]. As a result, only at high frequencies where the correction term of the impedance becomes negligible, the admittance agrees with the impedance and the propagation constant becomes purely imaginary and waves propagates with no loss above lossy ground [80]. However, when the ground is not perfectly conductive (non-ideal ground), the potential at the surface of the ground should not be considered as zero. Therefore, ground return corrections have to also be considered for shunt admittances of transmission lines [81].

In 1930's, Wise extended Carson's approach for relative permeability and permittivity of the earth greater than the unity [139] [137], and added the effects of transversal currents displacement for a single-conductor above an imperfect earth [138]. He referred to restricting assumptions of Carson's approach such as neglecting the polarization currents at low frequencies, and propagation of the wave with the velocity of the light and without attenuation. Kikuchi is the first author who proved the exact theory to Wise's solution by calculating the admittance correction term by means of a series expansion and pointing out the surface wave propagation at high frequencies [62]. Kikuchi's formula agrees with Wise's under the condition that the permeabilities of both medias are equal.

Sunde [113] and Arisumunandar [9] proposed similar image approximation [26] for the longitudinal effects of a transmission line. Hedman performed modal analysis on the admittance correction terms of Wise for high frequencies [56]. He concluded that the effects of the high relative dielectric constant of the earth are significant only for frequencies higher than $0.5 \mathrm{MHz}$, and when both earth resistivity and dielectric constant are high. Wedepohl further extended Wise correction term for a semi-infinite non-homogeneous earth [133] in terms of an infinite integral with no restriction on the permeabilities, permittivities, and resistivities. His numerical results showed that the integral becomes equivalent to that derived by Carson and Wise for the case of a semi-infinite homogeneous earth. However, considerable differences have been shown to occur in the stratified cases, when the earth-layer resistivities differ by a factor of 10 . Moreover, the capacitive nature of the earth was shown to be significant at very high frequencies due to displacement currents. 
In 1969, Wait proposed an exact solution of an implicit modal equation based on the full-wave approach of Sommerfeld integrals and Bessel functions in order to leave out of the Carson approximation for a thin, infinitely long wire parallel to an interface [125]. The formulation is rather general but very difficult to handle from a computational point of view. D'Amore presented an approximation to the exact solution of Wait for a single [21] and multi-conductor [22] transmission lines with further development to obtain simpler formulation [20]. Wedepohl and Efthymiadis developed a more general numerical technique to evaluate propagation constants for a single, lossless, infinitely long conductor above a lossy ground [129] [33]. This approach combines the vector potential as the sum of complementary transverse magnetic and transverse electric wave components for low and high frequencies showing similar results to Kikuchi's technique. The most obvious new feature of the results is the increase in the propagation loss due to a change in the reactive nature of the ground when high ground resistivities were involved. They realized that introducing a negative conductance term which is normally negligible, leads to a lossless propagation wave. This work was further enhanced by Nakagawa for a single [81] and multi-conductor [80] cases resulting in an approach with much smaller computation time than the solution of Efthymiadis. Nakagawa realized that in the case of ordinary lossy ground, admittance correction term does not have a significant impact in analyzing wave propagation at frequencies of interest to power engineers; whereas it produces remarkable changes in attenuation constants at high frequencies and for very lossy medium.

Pettersson generalized Sunde's image representation approach for the transverse effects, and achieved simple closed-form expressions for the axial propagation constant and characteristic impedance [94]. He further extended his formulation to include the vertical electric field between ground and wire [95]. Recently, Papadopoulos generalized Sunde's admittance correction approximation for overhead conductors above a two-layer earth [90].

\subsection{History of Frequency Domain Solutions}

As mentioned earlier, frequency domain solutions are used to assess the accuracy of the time domain line models since they do not approximate the frequency de- 
pendence of the line functions.

Pioneering work on power system transients simulation using numerical Fourier transforms was performed in 1965 [23]. This work addressed the Gibbs oscillations due to truncation of the integration range with infinite frequency of a continuoustime function. The authors introduced the use of the Lanczos' window to reduce Gibbs errors. They also referred to the aliasing errors in the time domain due to the discretization of the continuous variable in frequency. To reduce time aliasing, they proposed the Modified Fourier Transform (MFT) in which they smoothened the frequency response by adding a damping factor [24]. Wedepohl applied modal analysis to the MFT in the calculation of transients on multiconductor transmission lines [132] and later for underground and submarine cables [134]. The results obtained by Wedepohl at that time could not be attained with the available time domain methods and were used as benchmarks to enhance time domain line and cable models [101]. The computational efficiency of the MFT was enhanced by introducing the application of Fast Fourier Transform (FFT) [5] and by using non-uniform sampling [7] [60] [18] [45].

In 1978, Wilcox formulated the MFT methods in terms of the Laplace transform theory and introduced the term Numerical Laplace Transform (NLT) [136]. He also provided a criterion to calculate the associated damping factor. In 1982, Wedepohl further enhanced the numerical accuracy of the NLT by calculating the damping factor based on the number of samples [127]. The use of different window functions and the choice of damping factors for the NLT were studied in [75]. This reference also verified that the damping factor proposed by Wedepohl gives more accurate results than the one proposed by Wilcox, at the cost of amplifying the Gibbs oscillations and numerical errors in the tails of the waveforms.

Advances to include switching maneuvers, nonlinear and time-varying elements in the frequency domain solutions can be found in [131] [130] [79] [77] [122] [104] [76] [44] [109] [43] [42] [89] [17].

\subsection{History of Time Domain Line Models}

Time domain transmission line models are also referred to as "traveling-wave models" in which the voltages and currents at the two line ends are decomposed into 
incident and reflected waves. The behaviour of the line is described by the characteristic impedance which relates current waves to voltage waves and the propagation function which defines the delay and distortion of a wave traveling between the two line ends. Traveling-wave line models can be also classified into three types: Constant Parameters Line (CP-Line) model, modal domain models, and phase domain models. The CP-Line model was initially introduced by Bergeron in 1961 [10], and was the first model incorporated in the EMTP. The CP-Line is a simple, single-line constant-frequency model. Here, the line is treated as lossless with a pure time delay and a real characteristic impedance in the form of a dual Thevenin equivalent circuits. However, its distributed series resistance is added in lump form. The lumped resistances can be inserted throughout the line by dividing its total length into several sections. The main disadvantage of the CP-Line is that the relatively strong frequency dependence of the line parameters is not taken into account. Therefore, the CP-Line cannot adequately simulate the response of the line over the wide range of frequencies that are present in the signals during most transient conditions.

The theory of modal decomposition was introduced by Wedepohl in 1963 [128] and Hedman in 1965 [56] (independently from each other). This theory is based on the use of eigenvectors transformation matrices to decouple the physical system of an $\mathrm{N}$-phase multiconductor transmission line into $\mathrm{N}$-mathematically-equivalent decoupled single-phase circuits. Based on the modal decomposition theory and the $\mathrm{CP}$-Line model, one of the first frequency dependent multiconductor line models was proposed by Budner in 1970 [12]. He introduced the concept of weighting functions to model the frequency dependence of the line characteristic admittance and wave propagation functions. The weighting functions in this model are; however, highly oscillatory and difficult to evaluate with accuracy [68]. In an effort to improve Budner's model, in 1972 Snelson [111] introduced an analogous variable change to relate currents and voltages in the time domain. This idea was further developed by Meyer and Dommel [73] in 1974 and resulted in obtaining forward and backward travelling function from the weighted past history of the currents and voltages at both line ends using the convolution integral. This formulation represented a considerable improvement over other weighting function methods, and gave reliable results in many cases of transient studies [68]. On the other hand, the 
relatively time consuming process required to evaluate the convolution integrals at each time step of the solution and the less accurate response at low frequencies, including the nominal $60 \mathrm{~Hz}$ steady state, were the main drawbacks of this technique [68]. As a solution, in 1975 Semlyen and Dabuleanu [107] introduced an efficient formulation to synthesize the line functions with a low-order rational function approximation using complex exponentials. However, by allowing complex poles and zeros in the fitting functions, numerical oscillations were introduced in the response of line model.

J. Martí in 1981 [68] [69] introduced the FDLINE model that simplified frequency dependence modelling by fitting the amplitude of line functions with high order BAF rational approximations using negative poles and zeroes. This choice resulted in a minimum-phase realization (i.e., phase function is uniquely determined from the magnitude function) and, therefore, guarantees causality (i.e., the transfer function is equal to zero for $t<0$ ) and absolute numerical stability. In FDLINE, eigenvector transformation matrices are used to transform the original coupled phase coordinates into decoupled modal coordinates. FDLINE approximates the complex frequency dependent transformation matrices obtained with the classical MTL equations at one frequency (e.g., $1 \mathrm{kHz}$ ), and only takes the real part of the matrices. The original version of FDLINE was implemented in 1981-1983 in the Bonneville Power Administration (BPA) EMTP. This version was improved in the DCG/EPRI version developed in 1984-1986 [1]. Some of the specific improvements included: higher dynamics in the very low frequency region that solved encountered problems for very short lines, improved BAF algorithms that enhanced the overall accuracy of the fitting, and automatic error checking for short/open circuit conditions to optimize the single-frequency diagonalizing matrices used in this model. FDLINE is a simple, efficient, and reliable model that has been incorporated in most versions of the EMTP (e.g., ATP, Microtran, PSCAD, EMTP-RV, and OpalRT) and in real-time versions of the EMTP [32]. Even though FDLINE has been widely used, there has been a belief that because it uses a single real transformation matrix to convert between modal and phase quantities, the model may not be accurate enough for strongly asymmetrical line configurations [36] [38]. However, reference [70] showed that for switching surge studies in untransposed overhead lines, real constant transformation matrices can still give acceptable results within 
certain frequency limits. A number of line models have been proposed to remove the limitation of FDLINE of using a real constant transformation matrix as they will be briefly introduced next.

L. Martí in 1988 [71] proposed the fqLine model which synthesized the transformation matrices with BAF rational function approximations with real negative poles. This approach worked well in the case of underground cable where the conductors are very close to each other and to ground and the transformation matrices become strongly frequency dependent [72]. Fitting the transformation matrices with minimum-phase rational functions proved a difficult task. Here, the numerical difficulties of relaxing the constant transformation matrix condition for the overhead transmission lines began to emerge. Some of the terms that had to be fitted with rational functions could not be satisfactorily approximated using only negative poles and there was also the problem of modes switching along the frequency range [37].

In 1999, Tavares used a transformation matrix based on line geometry and Clarke transformation matrix to diagonalize frequency dependent multiphase transmission lines in the modal domain [114]. This model provides exact solution for ideally transposed lines and single-circuit three-phase horizontal lines and provides good approximation for non-transposed lines with vertical symmetry plane.

Recently, Gustavsen showed that the accuracy of FDLINE in two parallel overhead lines can be improved by modeling each line by an FDLINE and then adding the mutual coupling between the independent FDLINEs using rational functions [49]. However, this reference concluded that the proposed model seems to be computationally expensive particularly if the upper bandwidth passes $100 \mathrm{kHz}$.

The difficulties involved in the modal domain line models led to the development of alternative line models that do not use transformation matrices by working directly in phase coordinates. Examples of these models are the zLine [15], zCable [141], ARMA [88], [85], and other models based on the method of characteristics [84][102] [35].

Other phase domain models are based on the idempotent theory which was introduced by Cullen in 1990 [19] as "spectral decomposition". Wedepohl in 1993 [126] used this concept to synthesize the propagation function of transmission lines in phase coordinates. The idempotents are slightly better behaved functions than 
the eigenvectors of the modal transformation matrices; however, they are still frequency dependent. They also have the unique property to be scale-free, which is a key factor in solving the problem of normalization of the transformation matrices in fqLine model. Idempotent-based line models for the EMTP were first introduced by Castellanos and J. Martí in 1995 [14]. They were further developed by Marcano and J. Martí [65] [66]. In these models accuracy was sacrificed in order to use only minimum-phase functions for asymmetrical line configurations. Gustavsen and Semlyen in 1998 relaxed the requirement of minimum-phase in the synthesis of the idempotent coefficients to gain accuracy [52] [51] [54]. This work evolved in the ULM [74] in 1999 which is widely used in the EMTP community when frequency dependence of the transformation matrices is a concern. In developing the ULM, Gustavsen and Semlyen in 1999 introduced the VF rational approximation approach [55] with the improvement in [46] [50]. By allowing unstable poles and zeros in the VF, unlike BAF in FDLINE, ULM requires both the real and imaginary parts of the line functions to be fitted. Because of the higher burden of simulating the frequency dependence of the transformation matrices, ULM is considerably more expensive than FDLINE which is an important consideration in real time simulators. VF requires post-processing to achieve passivity [53] [16] [48] [27] [25] [108] and prevent numerical instability problems [63] [47]. Since physical systems with no sources have to be passive, a mathematical model without intrinsic passivity is not a good representation of reality and can lead to unexpected problems.

\subsection{Research Objectives and Anticipated Impacts}

The main objectives of this research are the followings:

1. Validation of FDLINE under asymmetrical line configurations

2. Development of an accurate frequency domain solution

3. Revisions to the classical MTL equations and development of a frequency dependent EMTP line model based on the revised equations 


\subsubsection{Validation of FDLINE Under Asymmetrical Line Configurations}

The FDLINE model has given reliable results in the EMTP community for many years. Even though FDLINE is simple, does not require passivity considerations, and has been implemented in real time simulators, the concern has remained of whether the model is accurate enough under asymmetrical line configurations. Many of the arguments for this conclusion have been based on using a single transformation matrix versus fitting the transformation matrix functions.

In Chapter 2, the accuracy of the FDLINE is compared with the ULM and NLT frequency domain solution for a variety of asymmetrical line configurations. By validating the accuracy of FDLINE in this chapter, this model will be used in Chapter 4 in the development of a line model based on the RMTL equations.

\subsubsection{Development of an Accurate Frequency Domain Solution}

In Chapter 3, derivation of the Discrete Time Fourier Series (DTFS) methodology is presented to solve electromagnetic transients in power systems with multiconductor lines. The proposed methodology is based on the correct specification of the time window and frequency window. Guidelines are provided for this set up. DTFS is a simpler solution than the most commonly used NLT due to not requiring to choose a damping factor and a windowing function to reduce aliasing and Gibbs errors typical of the frequency domain solutions; while it provides very accurate results.

\subsubsection{Revisions to the Classical MTL Equations and Development of a Frequency Dependent EMTP Line Model Based on the Revised Equations}

Chapter 4 addresses a physical inconsistency in the classical MTL equations that is the voltage waves and current waves do not travel together along the line. As a result, the characteristic impedance becomes a function of distance which contradictions Bergeron's theory.

To maintain the symmetry of propagation between the voltage waves and the current waves, a physical constraint is imposed on the MTL equations. Based on this condition the RMTL equations are proposed. As opposed to the MTL equations that require complex frequency dependent transformation matrices for their diag- 
onalization, the RMTL equations can be diagonalized using a single real constant transformation matrix.

A Frequency-Dependent Line Model (FDLM) is proposed based on the RMTL equations. The accuracy of FDLM is compared to FDLINE, ULM, and DTFS frequency domain solution. 


\section{Chapter 2}

\section{Accuracy of FDLINE Under Asymmetrical Line Configurations}

This chapter first reviews the classical MTL equations for an $N$-conductor overhead transmission line with ground return on which the existing line models are based. This includes calculation of per unit length impedance and admittance matrices as well as derivation of the transformation matrices to diagonalize the wave functions. Next, well-known time domain and frequency domain solutions to the MTL equations are briefly discussed. Finally, the simulation results obtained with FDLINE and ULM line models in the EMTP are presented for a variety of asymmetrical line configurations, including single-circuit, double-circuit and parallel lines. The results are compared with a conventional NLT reference solution. The comparisons include open and short circuit conditions during the transient and steady state periods.

The MTL equations will be used in Chapter 3 in the development of the DTFS algorithm, and in Chapter 4 to propose the RMTL equations and develop the FDLM model. 


\subsection{Classical MTL Equations}

\subsubsection{Per Unit Length $Z$ and $Y$ Calculation}

In Fig. 2.1, a segment $\Delta x$ of a long uniform transmission line in a uniform medium is shown. In general, the equations are derived for $N$ parallel conductors above a common ground return. The equations relating voltages and currents in this line can be derived in the frequency domain in terms of series impedance and shunt admittance matrices.

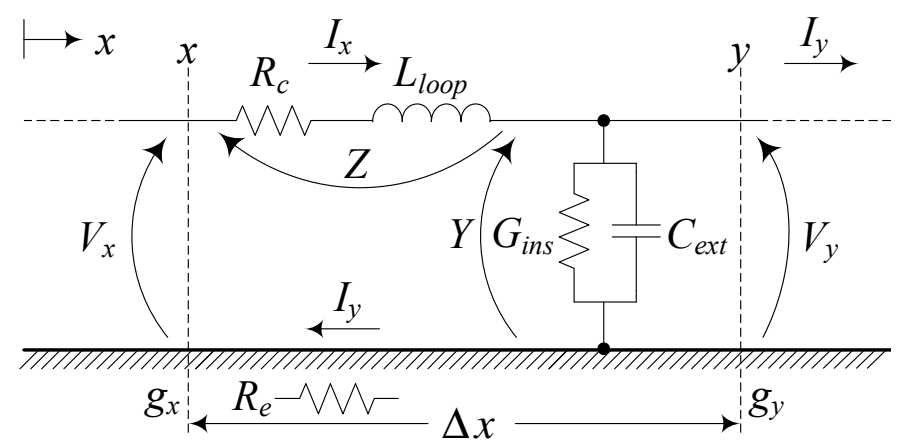

Figure 2.1: Transmission line segment $\Delta x$ (only one conductor with respect to ground is shown).

The series impedance and shunt admittance matrices in Fig. 2.1 are symmetrical and their elements are complex numbers. Preserving this symmetry is one of the physical constraints imposed in this research. Voltages are measured from conductors to a single common ground. Currents in the conductors flow forward in the $x$ direction and return through the common ground path. The voltage and current drops along the line conductors are given by,

$$
\mathbf{V}_{x}-\mathbf{V}_{y}=\mathbf{Z I} \quad, \quad \mathbf{I}_{x}-\mathbf{I}_{y}=\mathbf{Y} \mathbf{V}
$$

The real part of the impedances in $\mathbf{Z}$ consists of the internal resistance of the conductors $\mathbf{R}_{c}$ plus the resistance of the ground return path $\mathbf{R}_{e}$. The imaginary part of $\mathbf{Z}$ consists of the inductances of the loops $\mathbf{L}_{\text {loop }}$ formed by the conductors and ground. In the normal EMTP formulation, the real part of the admittances matrix $\mathbf{Y}$ consists of the losses along the insulator strings $\mathbf{G}_{i n s}$, and the imaginary part 
consists of the external conductor capacitances $\mathbf{C}_{\text {ext }}$. The series impedance and shunt admittance matrices are then given by

$$
\begin{aligned}
& \mathbf{Z}=\left(\mathbf{R}_{c}+\mathbf{R}_{e}\right)+j \omega \mathbf{L}_{\text {loop }} \\
& \mathbf{Y}=\mathbf{G}_{\text {ins }}+j \omega \mathbf{C}_{\text {ext }}
\end{aligned}
$$

In general, all elements of the matrices in (2.2) can be frequency dependent. In normal EMTP transmission line modelling, however, $\mathbf{G}_{\text {ins }}$ and $\mathbf{C}_{\text {ext }}$ are assumed constant.

In (2.2), $\mathbf{R}_{c}$ and $\mathbf{G}_{\text {ins }}$ are diagonal matrices. $R_{c}$ is calculated using Bessel functions to account for the skin effect. In the EMTP simulation tools (e.g., Microtran, PSCAD, and EMTP-RV), $R_{c}$ is calculated knowing the outer diameter, thickness ratio, and DC resistance of an Aluminum Conductor Steel Reinforced (ACSR) conductor. The value of the $G_{i n s}$ is in the order of the $\left(10^{-9}\right)$ 's $\mathrm{S} / \mathrm{km}$ [39] which is normally small compared to the other system parameters. However, ignoring this parameter causes a relative asymmetrical property between the $\mathbf{Z}$ and $\mathbf{Y}$ matrices, even though negligible.

$\mathbf{R}_{e}, \mathbf{L}_{\text {loop }}$, and $\mathbf{C}_{\text {ext }}$ are full matrices which are calculated using the concept of complex penetration depth [26].

The diagram in Fig. 2.2 illustrates Lord Kelvin's method of images where for ideal ground each line conductor at height $h_{i}$ has a corresponding image in the ground at depth $h_{i}$, and the actual ground can be replaced by the conductors' images.

In an approximate solution, that illustrates the nature of the problem, the complex penetration depth method of [26] can be used to adjust the height of the conductors and the depth of their images (Fig. 2.2) to take into account non-ideal ground. The adjusting complex penetration depth value $\bar{p}$ is given by

$$
\bar{p}=\sqrt{\frac{\rho}{j \omega \mu}}
$$

In (2.3), $\rho$ is the earth resistivity, $\omega$ is $2 \pi f$ where $f$ is the frequency and $\mu$ is the permeability of the ground. For the air and the ground $\mu=\mu_{0}=4 \pi \times 10^{-7}$ in $\mathrm{H} / \mathrm{m}$. The radii of conductors is taken as $r_{i e q}$ to correct for the internal skin effect. 


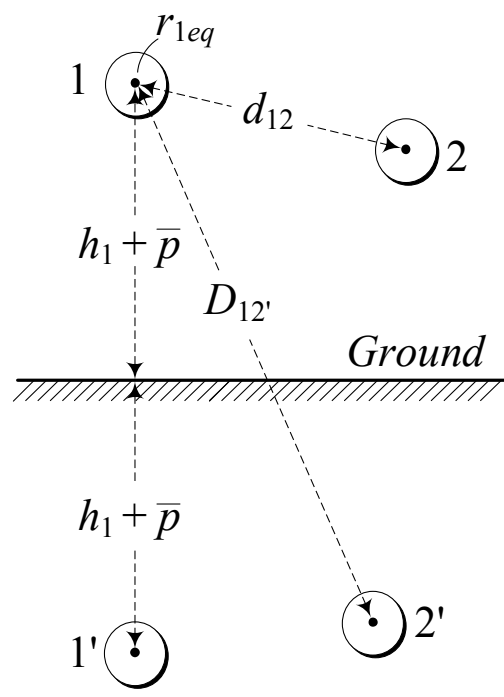

Figure 2.2: Conductors above non-ideal ground using the method of images and complex penetration depth.

In Fig. 2.2 we show two conductors and their ground return images. In general we will have $N$ physical conductors with $N$ images. The classical MTL line equations, however, are derived for $N$ conductors above a single ground return. Mapping the system of conductors and images in Fig. 2.2 to the system of conductors with a common ground return, there is a loss of dimensionality of $N-1$. This loss of information leads to the inconsistencies in the calculated values for $\mathbf{R}_{e}$ which will be further discussed in Section 4.3.

To calculate the values of $\mathbf{R}_{e}$ using the method of images, we can define a complex loop inductance $\mathbf{L}_{\text {loop }}^{\prime}$ that takes into account the inductance and the resistance of the path formed by the conductor and the earth return. The self element $L_{\text {loop } 11}^{\prime}$ in Fig. 2.2 is calculated in

$$
L_{\text {loop } 11}^{\prime}=\frac{\mu}{2 \pi} \ln \frac{2\left(h_{1}+\bar{p}\right)}{r_{1 e q}}
$$

The real part of the $L_{\text {loop } 11}^{\prime}$ defines the self inductance of the loop, while the imaginary part corresponds to the self earth resistance given by 


$$
\begin{aligned}
L_{\text {loop } 11} & =\mathfrak{R}\left(L_{\text {loop } 11}^{\prime}\right) \\
R_{e 11} & =j \omega\left(j \mathfrak{I}\left(L_{\text {loop } 11}^{\prime}\right)\right)=-\omega \mathfrak{I}\left(L_{\text {loop } 11}^{\prime}\right)
\end{aligned}
$$

The mutual element $L_{\text {loop } 12}^{\prime}$ in Fig 2.2 is calculated in

$$
L_{\text {loop } 12}^{\prime}=\frac{\mu}{2 \pi} \ln \frac{D_{12^{\prime}}}{d_{12}}
$$

In (2.7), $D_{12^{\prime}}$ is the distance from conductor 1 to the image of conductor $2, d_{12}$ is the physical distance from conductor 1 to conductor 2 .

The mutual elements for inductance loop and earth resistance are given by

$$
\begin{aligned}
L_{\text {loop } 12} & =\mathfrak{R}\left(L_{\text {loop } 12}^{\prime}\right) \\
R_{e 12} & =j \omega\left(j \mathfrak{I}\left(L_{\text {loop } 12}^{\prime}\right)\right)=-\omega \mathfrak{I}\left(L_{\text {loop } 12}^{\prime}\right)
\end{aligned}
$$

For the $\mathbf{Y}$ matrix of (2.2), the matrix of capacitances $\mathbf{C}_{\text {ext }}$ is obtained from the matrix of potential coefficients $\mathbf{P}_{\text {ext }}$ as

$$
\mathbf{C}_{e x t}=\mathbf{P}_{e x t}^{-1}
$$

Following the TEM condition in the classical MTL equations, the capacitance is assumed to be equal to its electrostatic value (with the charges on the outside of the conductor and on the surface of the earth). Since no transversal losses are considered, the values of $\mathbf{P}_{\text {ext }}$ and $\mathbf{C}_{\text {ext }}$ are real. The diagram of Fig. 2.2, with $\bar{p}=$ 0 , can be used to represent this electrostatic condition and calculate the potential coefficients. The self and mutual elements of real matrix $\mathbf{P}_{\text {ext }}$ in the example of Fig 2.2 are calculated in

$$
P_{\text {ext } 11}=\frac{1}{2 \pi \varepsilon} \ln \frac{2 h_{1}}{r_{1 e x t}} \quad, \quad P_{\text {ext } 12}=\frac{1}{2 \pi \varepsilon} \ln \frac{D_{12}}{d_{12}}
$$

In (2.11), $r_{\text {lext }}$ is the external radius of conductor 1 in Fig. 2.2 and $D_{12}$ is the distance from conductor 1 to the image of conductor 2 when the ground penetration depth is zero. For the air and the ground $\varepsilon=\varepsilon_{0}=8.85 \times 10^{-12}$ in $\mathrm{F} / \mathrm{m}$. 


\subsubsection{Diagonalization of the MTL Equations}

Analogous formulas can be written for the voltage drops in the series impedances between sections $x$ and $y$ and current drops across the shunt admittances, for conductors 1 and 2 in Fig. 2.1. Assuming $\mathbf{Z}$ is in $\Omega / \mathrm{m}$ and $\mathbf{Y}$ is in $S / m$, and making the length of the line segment $\Delta x$ go to zero, we obtain first order differential equations for the voltage and current drops as a function of the distance $x$ down the line,

$$
-\frac{\partial \mathbf{V}}{\partial x}=\mathbf{Z I} \quad, \quad-\frac{\partial \mathbf{I}}{\partial x}=\mathbf{Y} \mathbf{V}
$$

The negative signs in (2.12) are due to the assumed positive direction for the $x$ in Fig. 2.1. Combining voltages and currents in (2.12), we obtain second order differential equations for the propagation of voltage and current as a function of the distance $x$ down the line,

$$
\frac{\partial^{2} \mathbf{V}}{\partial x^{2}}=(\mathbf{Z Y}) \mathbf{V} \quad, \quad \frac{\partial^{2} \mathbf{I}}{\partial x^{2}}=(\mathbf{Y Z}) \mathbf{I}
$$

Due to the duality of electric and magnetic field in the propagation process, symmetry conditions need to be preserved in the solution of the propagation equations. Since the quantities in (2.13) are matrices, we need, in general, to preserve the order of the multiplications. It is then assumed in the classical MTL solution (e.g., [126][93]) that

$$
\mathbf{Z Y} \neq \mathbf{Y Z}
$$

As we will show in Chapter 4, this assumption will lead to a physical contradiction in the symmetry of the solution. The solution of (2.13) can be written as

$$
\begin{gathered}
\mathbf{V}(x)=\mathbf{V}_{f}(x)+\mathbf{V}_{b}(x)=\mathbf{V}_{f k} e^{-\gamma_{v} x}+\mathbf{V}_{b k} e^{+\gamma_{\mathbf{V}} x} \\
\mathbf{I}(x)=\mathbf{I}_{f}(x)+\mathbf{I}_{b}(x)=\mathbf{I}_{f k} e^{-\gamma_{1} x}+\mathbf{I}_{b k} e^{+\gamma_{\mathbf{I}} x}
\end{gathered}
$$

The wave propagation constants $\gamma_{V}$ and $\gamma_{I}$ are found from the solutions of (2.13),

$$
\gamma_{V}=\sqrt{\mathbf{Z Y}}=\sqrt{\left(\mathbf{R}_{c}+\mathbf{R}_{e}+j \omega \mathbf{L}_{\text {loop }}\right)\left(\mathbf{G}_{\text {ins }}+j \omega \mathbf{C}_{\text {ext }}\right)}
$$




$$
\gamma_{I}=\sqrt{\mathbf{Y Z}}=\sqrt{\left(\mathbf{G}_{\text {ins }}+j \omega \mathbf{C}_{\text {ext }}\right)\left(\mathbf{R}_{c}+\mathbf{R}_{e}+j \omega \mathbf{L}_{\text {loop }}\right)}
$$

Without any further restrictions in the MTL equations, and except for the case of a balanced line or a single-phase line, $\gamma_{V}$ and $\gamma_{I}$ in (2.17) and (2.18) are in general different.

The voltage and current waves in (2.15) and (2.16) have two components: the forward waves " $f$ " that travel from left to right in the diagram of Fig. 2.1, and the backward waves " $b$ " that travel from right to left in this diagram.

Assuming a semi-infinite line, where only forward voltage and current waves exist in (2.15) and (2.16), the voltage waves and the current waves are related by the characteristic impedance (2.19)-left. Similarly, the backward voltage and current waves are related as shown in (2.19)-right (the minus sign here indicates that the backward currents flow from right to left),

$$
\mathbf{V}_{f}(x)=\mathbf{Z}_{c} \mathbf{I}_{f}(x) \quad, \quad \mathbf{V}_{b}(x)=-\mathbf{Z}_{c} \mathbf{I}_{b}(x)
$$

The characteristic impedance matrix $\mathbf{Z}_{c}$ can be found by replacing (2.19) in (2.12) giving

$$
\mathbf{Z}_{c}=\mathbf{Y}^{-1} \sqrt{\mathbf{Y Z}}
$$

where $\mathbf{Z}$ and $\mathbf{Y}$ are defined in (2.2).

For single-phase lines, $\mathbf{Z}$ and $\mathbf{Y}$ are scalar numbers and their product is commutative. In this case, $\gamma_{V}$ and $\gamma_{I}$ are the same. This is also the case for fully transposed ("balanced") multiconductor lines that can be diagonalized by symmetrical components and other akin transformations. However, for untransposed multi-phase lines, $\gamma_{V}$ and $\gamma_{I}$ are different. This is the currently held assumption in the MTL equations.

When modal analysis is used to transfer the line equations from phase to modal coordinates (Wedepohl, 1963 [128] and Hedman 1965 [56]), two transformation matrices $\mathbf{T}_{V}$ and $\mathbf{T}_{I}$ are defined, one for the voltages and one for the currents. Using subscript $p h$ for the original coupled phase quantities and subscript $m$ for the decoupled modal quantities, 


$$
\mathbf{V}_{p h}=\mathbf{T}_{V} \mathbf{V}_{m} \quad, \quad \mathbf{I}_{p h}=\mathbf{T}_{I} \mathbf{I}_{m}
$$

Matrix $\mathbf{T}_{V}$ diagonalizes the product $\mathbf{Z Y}$ while matrix $\mathbf{T}_{I}$ diagonalizes the reverse product $\mathbf{Y Z}$,

$$
\mathbf{Z} \mathbf{Y}_{m}=\mathbf{T}_{V}^{-1}\left(\mathbf{Z}_{p h} \mathbf{Y}_{p h}\right) \mathbf{T}_{V} \quad, \quad \mathbf{Y} \mathbf{Z}_{m}=\mathbf{T}_{I}^{-1}\left(\mathbf{Y}_{p h} \mathbf{Z}_{p h}\right) \mathbf{T}_{I}
$$

In the modal domain, the voltage propagation $\mathbf{Z} \mathbf{Y}_{m}$ is equal to the current propagation $\mathbf{Y Z} \mathbf{Z}_{m}$.

Since the transformation matrices contain complex numbers, they are prone to mode switching that has to be corrected. A well-known solution to this problem was proposed by Wedepohl with the application of Newton-Raphson algorithm [135].

The decoupled modal series impedances and shunt admittances are calculated from

$$
\mathbf{Z}_{m}=\mathbf{T}_{V}^{-1} \mathbf{Z}_{p h} \mathbf{T}_{I} \quad, \quad \mathbf{Y}_{m}=\mathbf{T}_{I}^{-1} \mathbf{Y}_{p h} \mathbf{T}_{V}
$$

It will be argued in Chapter 4 that the constraint of collocation of voltage waves and current waves will require $\gamma_{V}$ and $\gamma_{I}$ to be equal in the phase domain, regardless of the asymmetry of the system of conductors (vertical, horizontal, double circuit, etc.). It will also be shown that this condition can be achieved with a single real transformation matrix $\mathbf{T}$ which is basically constant over the entire frequency range.

\subsection{Time Domain and Frequency Domain Solutions to the MTL Equations}

\subsubsection{FDLINE Solution to the MTL Equations}

In the FDLINE model, modal transformation matrices are used to decouple the $N$ coupled transmission lines to $N$-decoupled circuits. Each circuit is represented by a modal characteristic impedance $Z_{c m}$ and a modal propagation function $\gamma_{m}$ calculated the at each frequency from

$$
Z_{c m}=\sqrt{\frac{Z_{m}}{Y_{m}}} \quad, \quad \gamma_{m}=\sqrt{Z_{m} Y_{m}}
$$


At each decoupled circuit, the voltage and current waves are calculated in

$$
\begin{gathered}
V(x)=V_{f k} e^{-\gamma_{m} x}+V_{b k} e^{+\gamma_{m} x} \\
I(x)=I_{f k} e^{-\gamma_{m} x}+I_{b k} e^{+\gamma_{m} x}
\end{gathered}
$$

The voltage and current waves are related by the characteristic impedance in

$$
V(x)=V_{f k} e^{-\gamma_{m} x}+V_{b k} e^{+\gamma_{m} x}=Z_{c m}\left(I_{f k} e^{-\gamma_{m} x}+I_{b k} e^{+\gamma_{m} x}\right)
$$

Combining (2.25) (2.26) as in [69], we obtain the forward perturbation wave

$$
V(x)+Z_{c m} I(x)=\left(V_{k}+Z_{c m} I_{k}\right) e^{-\gamma_{m} x}
$$

Note that in FDLINE [69], (2.28) can only be written in terms of modal decoupled modes. Figure 2.3 illustrates FDLINE equivalent circuit at each mode. In (2.28), with $x=0$ for the sending-end $k$ of the line of Fig. 2.3, and $x=\ell$ for the receiving end $n$, one obtains the voltage and current relationship for the line equivalent circuit at node $n$,

$$
V_{n}+Z_{c m} I_{n}=\left(V_{k}+Z_{c m} I_{k}\right) e^{-\gamma_{m} \ell}
$$

The circuit of Fig. 2.3-right results from (2.29) after reversing the direction of the current. A similar analysis can be followed to obtain the equivalent circuit for the line as seen from node $k$ (Fig. 2.3-left).

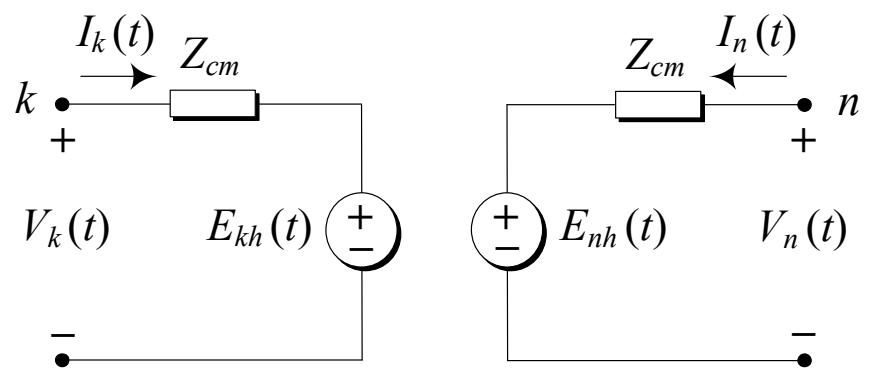

Figure 2.3: FDLINE modal equivalent circuit.

The history sources in Fig. 2.3 are given by

$$
E_{n h}=\left(V_{k}+Z_{c m} I_{k}\right) e^{-\gamma_{m} \ell} \quad, \quad E_{k h}=\left(V_{n}+Z_{c m} I_{n}\right) e^{-\gamma_{m} \ell}
$$


In FDLINE model, modal frequency dependent $Z_{c m}$ and $e^{-\gamma_{m} \ell}$ in the circuit of Fig. 2.3 are synthesized by means of BAF rational function approximation using negative poles and zeros to guarantee a passive realization.

The BAF algorithm is based on an adaptation of the simple concept of asymptotic fitting of the magnitude function, first introduced by Bode. The magnitude is plotted as a function of the logarithm of the frequency. The basic principle of the procedure is to trace the approximated curve by straight-line segments. These segments may be either horizontal or have a slope that is a multiple of $20 \mathrm{~dB} / \mathrm{dec}$. The points of change of slope (corners or breaking points) define the poles and zeros of the rational approximating function [68].

A major advantage of BAF over the other curve fitting algorithms which use complex poles (e.g., VF) is the process used to determine the time delay associated with the propagation modes [1] [69]. Because BAF uses only negative poles and zeroes, the resulting rational approximation is minimum phase-shift [92]. This means that the real and imaginary parts are locked in a single function and the phase is uniquely defined by the fit of the magnitude. For the propagation functions, the rational fit has the same magnitude as the data function and the phase has a shift of $\omega \tau$ due to the time delay $\tau$ of the propagating mode. In FDLINE of [1] [69], this time delay is determined by shifting the fitted function a for best correlation with the original function. Since the original function is physical, it has to be minimumphase-shift with a time delay and the BAF procedure assures the physical properties of the fit. This correlation procedure maintaining the physical constraints is not possible when the fitting is not restricted to minimum-phase functions, like in the case of VF [55].

After approximating $Z_{c}$ by some poles and zeros, then it is simulated by a series of $\mathrm{RC}$ parallel circuits. Using the trapezoidal integration rule, the $\mathrm{RC}$ networks are expressed in the form an equivalent resistance in series with a history voltage source. Synthesis of the $e^{-\gamma \ell}$ results in two uncoupled parallel history voltage source derived from the Inverse Laplace Transform of the partial fraction expression of the approximated polynomials. The model can then be easily incorporated into a time domain solution simply in the form of a constant resistance in parallel with a current source evaluated at each time step of the solution.

Finally, the voltage and current of interest will be transferred from the modal 
domain back to the phase domain using (2.21). As addressed in the literature, FDLINE approximates the transformation matrices in one frequency (e.g., $1 \mathrm{kHz}$ ) and takes only the real part of the matrices.

The total operations count for FDLINE to simulate an $N$ conductor system with $P_{Z_{c}}$ number of poles for fitting $Z_{c}$ and $P_{A_{p}}$ number of poles for fitting $e^{-\gamma \ell}$ is given by

$$
N_{\text {fdline }}=\left(N^{2}+\sum_{1}^{N} P_{Z_{c}}\right)+\left(N^{2}+\sum_{1}^{N} P_{A_{p}}\right)
$$

\subsubsection{ULM Solution to the MTL Equations}

The ULM is a direct phase domain frequency dependent line model which is fundamentally based on the idempotent decomposition (introduced as "column-wise" technique in [74]).

Idempotent coefficients are matrices derived from the column-row multiplication of the modal transformation matrix and its inverse. For a function $\mathbf{A}$, the relationship between the phase domain and modal domain are defined in

$$
\begin{aligned}
& \mathbf{A}_{m}=\mathbf{T}^{-1} \mathbf{A}_{p h} \mathbf{T} \\
& \mathbf{A}_{p h}=\mathbf{T} \mathbf{A}_{m} \mathbf{T}^{-1}
\end{aligned}
$$

Equation (2.33) can be rewritten in terms of the constituent columns for $\mathbf{T}$ and constituent rows for $\mathbf{T}^{-1}$ for an $N$-phase multiconductor transmission lines in

$$
\mathbf{A}_{p h}=\left[\begin{array}{llll}
\mathrm{C}_{1} & C_{2} & \cdots & C_{N}
\end{array}\right]\left[\begin{array}{cccc}
\mathrm{a}_{11} & 0 & \cdots & 0 \\
0 & a_{22} & \cdots & 0 \\
\vdots & \vdots & \ddots & \vdots \\
0 & 0 & \cdots & a_{N N}
\end{array}\right]\left[\begin{array}{c}
\mathrm{R}_{1} \\
R_{2} \\
\vdots \\
R_{N}
\end{array}\right]=\sum_{i=1}^{N} a_{i i}\left(\mathbf{C}_{i} \mathbf{R}_{i}\right)
$$

The ULM uses VF algorithm to synthesize the modal line functions $Y_{c}$ and $e^{-\gamma \ell}$ and the idempotent coefficients matrices. VF method is a robust reformulation of the Sanathanan-Koerner iteration [103] using rational basis functions (partial fractions) instead of polynomials and pole relocation instead of weighting [57]. VF 
is based on iteratively relocating an initial pole set to better locations [46]. Unlike the BAF, VF requires both the magnitude and phase angle of the mode to be known before the fitting can be done. Thus, the time constant for back-winding must be pre-calculated.

In this model, VF was initially used to fit $Y_{c}$ column-wise using an identical set of poles. Since $Y_{c}$ has no time delay, instead of fitting the sum of modes in phase domain using suitable sets of poles, fitting is done on the sum of the diagonal elements of $Y_{c}$. By taking the modal traveling time delays into account, the propagation matrix is fitted in the phase domain. The resulting poles and time delays are then used for fitting $e^{-\gamma \ell}$ in the phase domain, under the assumption that all poles contribute to all elements of $e^{-\gamma \ell}$. The unknown residues are then calculated by solving an over-determined system of linear equation as a least squares problem. As all elements in $e^{-\gamma \ell}$ get identical poles, a column-wise realization [88] can be used which gives increased computational efficiency for the time domain simulation.

The total operations count for ULM to simulate an $N$ conductor system with $P_{Y_{c}}$ number of common poles for fitting $\mathbf{Y}_{c}$ and $P_{A_{p}}$ number of poles for fitting $e^{-\gamma \ell}$ for $N$ idempotents is given by

$$
N_{U L M}=\left(N^{2} \times P_{Y_{c}}\right)+\left(N^{2} \times \sum_{1}^{N} P_{A_{p}}\right)
$$

\subsubsection{NLT Solution to the MTL Equations}

The NLT implemented in this chapter follows the formulations in [75]. The NLT method solves the system directly in the frequency domain using nodal analysis. The current sources are converted from time to frequency, the admittance matrix is sampled in frequency, the system is solved, and finally the network voltages are transferred from frequency to time. To relate the time and frequency domains, NLT requires to define a time window of width $T_{c}$ and a frequency window of width $f_{c}$ with discrete samples. The time step size and the frequency step size are given by

$$
\Delta t=\frac{1}{f_{c}} \quad, \quad \Delta f=\frac{1}{T_{c}}
$$

The number of sample points (solution points) $N_{s}$ is determined by 


$$
N_{s}=\frac{T_{c}}{\Delta t}=\frac{f_{c}}{\Delta f}
$$

Two sampling scheme are introduced in [75] to discretize the parameters in the frequency domain : regular sampling and odd sampling,

$$
\Delta \omega=2 \pi k \Delta f(\text { regular }) \quad, \quad \Delta \omega=\pi(2 k+1) \Delta f(\text { odd })
$$

where, $k=0,1,2, \ldots, N_{s}-1$.

In addition to the discrete frequency, the Laplace variable $s$ in the NLT includes a constant damping factor $\sigma$ given in,

$$
s=\sigma+j \Delta \omega
$$

The factor $\sigma$ is chosen to provide artificial damping to the time signal. By dampening the time signal, the signal will fit into the time window $T_{c}$ and the time aliasing problem due to the discretization of the admittance matrix $\mathbf{Y}(s)$ is reduced. There are three commonly used criteria for the calculation of the damping factor: Wilcox criteria $\sigma_{1}$ [136], Wedepohl criteria $\sigma_{2}$ [127], and error criteria $\sigma_{3}$ [43]:

$$
\sigma_{1}=2 \Delta \omega \quad, \quad \sigma_{2}=\frac{\ln N_{s}^{2}}{T_{c}} \quad, \quad \sigma_{3}=-\frac{\log \varepsilon}{T_{c}}
$$

where $\varepsilon$ is the error tolerance.

After identifying a time window and a frequency window, the input current sources are transferred from time to frequency using the continuous Laplace transform.

$$
I(s)=\left.\int_{0}^{\infty} i(t) e^{-s t} d t\right|_{s=\sigma+j \Delta \omega}
$$

Then, the system is solved using nodal analysis one frequency at a time in

$$
\mathbf{V}(s)=\mathbf{Y}^{-1}(s) \mathbf{I}(s)
$$

In (2.42), $\mathbf{V}$ is the calculated vector of output node voltages, $\mathbf{Y}$ is the matrix of admittances evaluated at each frequency, and $\mathbf{I}$ is the injected current sources in the Laplace domain. To transfer the output network voltages from frequency to time, 
NLT requires to use a boosting factor $C_{n}$ and a weighting or windowing function (filter) $W$ with the Inverse Fast Fourier Transform (IFFT) as shown in

$$
v(t)=\Re\left(C_{n} \operatorname{IFFT}\{V(s) W(s)\}\right)
$$

In (2.43), the real part of the signal is taken to eliminate the noise. The boosting factor $C_{n}$ is used to reverse the effect of the original artificial damping. $C_{n}$ for the regular sampling and odd sampling is calculated in

$$
C_{n}=\frac{1}{\Delta t} e^{\sigma n \Delta t}(\text { regular }) \quad, \quad C_{n}=\frac{2}{\Delta t} e^{\left(\sigma \Delta t+j \frac{\pi}{N_{s}}\right) n}(\text { odd })
$$

where, $n=0,1,2, \ldots, N_{s}-1$.

In (2.43), windowing function $W$ is usually applied to the NLT to attenuate the Gibbs oscillations produced by the truncation of the frequency range. Lanczos and Hanning are examples of such windowing functions [43].

To incorporate multiconductor transmission lines in the NLT solution, a coupled exact- $\pi$ model are used as shown in Fig. 2.4.

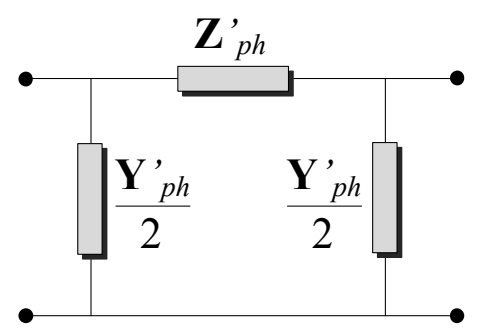

Figure 2.4: Coupled exact- $\pi$ model. Frequency domain equivalent-circuit for multiconductor transmission line.

To form this model, the series impedances $Z_{m}^{\prime}$ and shunt admittances $Y_{m}^{\prime}$ of the decoupled exact- $\pi$ circuits are calculated in (2.45) by correcting the modal per unit length impedances and admittances (2.23) for the length $\ell$ and frequency. The correction is performed by multiplying $Z_{m}$ and $Y_{m}$ by factors $k_{z}$ and $k_{y}$ in 


$$
\begin{aligned}
& Z_{m}^{\prime}=k_{z} Z_{m} \ell, \quad Y_{m}^{\prime}=k_{y} Y_{m} \ell \\
& k_{z}=\frac{\sinh \left(\gamma_{m} \ell\right)}{\gamma_{m} \ell} \quad, \quad k_{y}=\frac{\tanh \left(\frac{\gamma_{m} \ell}{2}\right)}{\frac{\gamma_{m} \ell}{2}}
\end{aligned}
$$

where $\gamma_{m}$ is the modal propagation function calculated in (2.24).

The coupled exact- $\pi$ model is obtained by transferring $Z_{m}^{\prime}$ and $Y_{m}^{\prime}$ from modal domain to phase domain in

$$
\mathbf{Z}_{p h}^{\prime}=\mathbf{T}_{V} \mathbf{Z}_{m}^{\prime} \mathbf{T}_{I}^{-1} \quad, \quad \mathbf{Y}_{p h}^{\prime}=\mathbf{T}_{I} \mathbf{Y}_{m}^{\prime} \mathbf{T}_{V}^{-1}
$$

It is important to note that the frequency variable to calculate the line parameters in the Laplace domain is $s=\sigma+j \Delta \omega$.

\subsection{Case Studies and Simulation Conditions}

In this section, six line configurations are introduced to compare the solutions given by FDLINE, ULM, and NLT:

Test 1: Single-phase line.

Test 2: Three-phase single-circuit horizontal line [66].

Test 3: Three-phase single-circuit vertical line [34].

Test 4: Three-phase single-circuit delta line [34].

Test 5: Three-phase double-circuit one-tower delta line [66].

Test 6: Three-phase double-circuit two-tower horizontal line [49].

Single-circuit lines are considered untransposed to test FDLINE under asymmetrical line configurations and double circuit lines are chosen from the references that previously criticized FDLINE for inaccuracy [66] [49]. In the test cases, only one conductor per bundle is considered in order to leave out the effect of the various bundle modelling approaches used in different software packages.

In Figs. 2.5 to 2.10 the line geometries and the terminal conditions of the conductors (open or shorted) are shown. The line length are selected based on the source voltage level. As expected, the transients last longer in short lines due to the higher perturbations caused by forward and backward waves travelling along the 
short length.
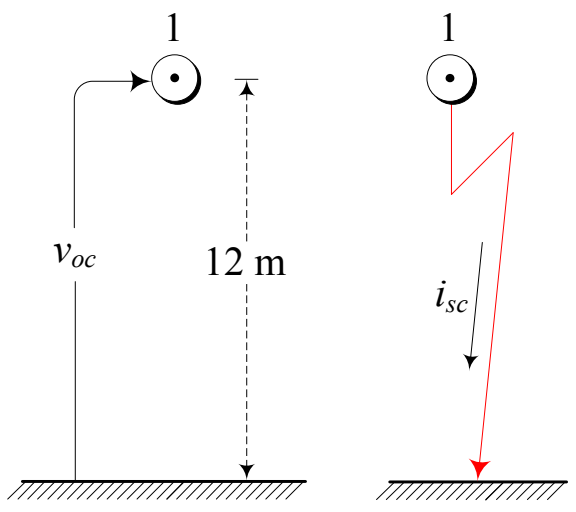

\begin{tabular}{l} 
Phase wires: \\
$d=2.5(\mathrm{~cm})$, \\
$R_{d c}=0.0576(\Omega / \mathrm{km})$, \\
Ratio $=0.5$, \\
Source: \\
$V_{s}=93.89 \cos (2 \pi 60 t)(\mathrm{kV})$, \\
$R_{s}=0.5(\Omega), L_{s}=0.03(\mathrm{H})$, \\
Ground: \\
$\rho=100(\Omega . \mathrm{m})$, \\
$\mu=1$, \\
Length: $150(\mathrm{~km})$, \\
$\Delta t=5 \mu \mathrm{s}$. \\
\hline
\end{tabular}

Figure 2.5: Single-phase transmission line (open and shorted).

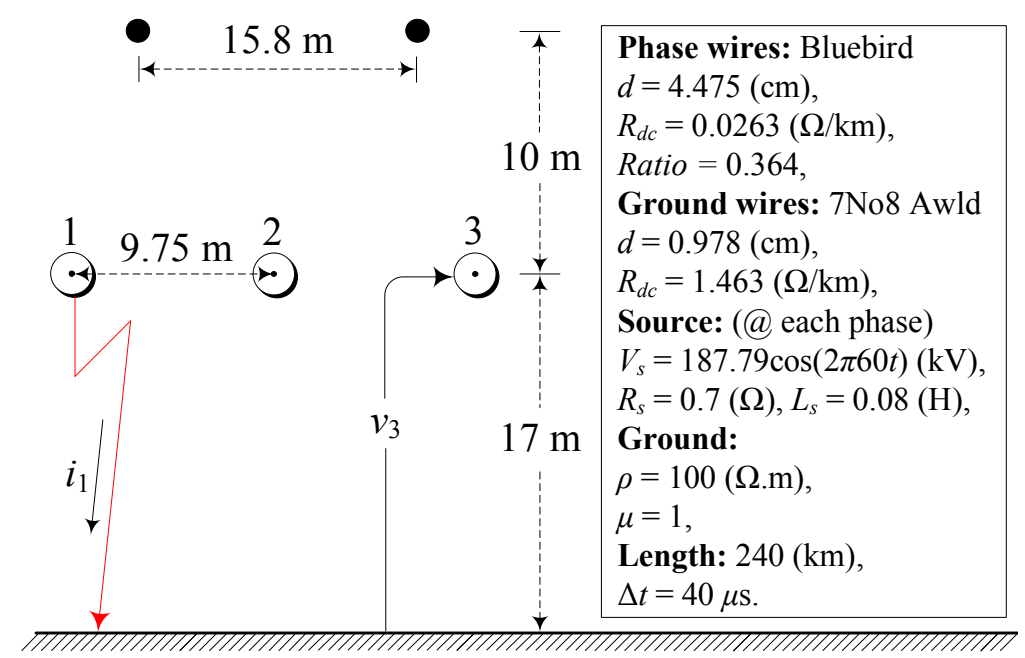

Figure 2.6: Three-phase single-circuit horizontal transmission line. 


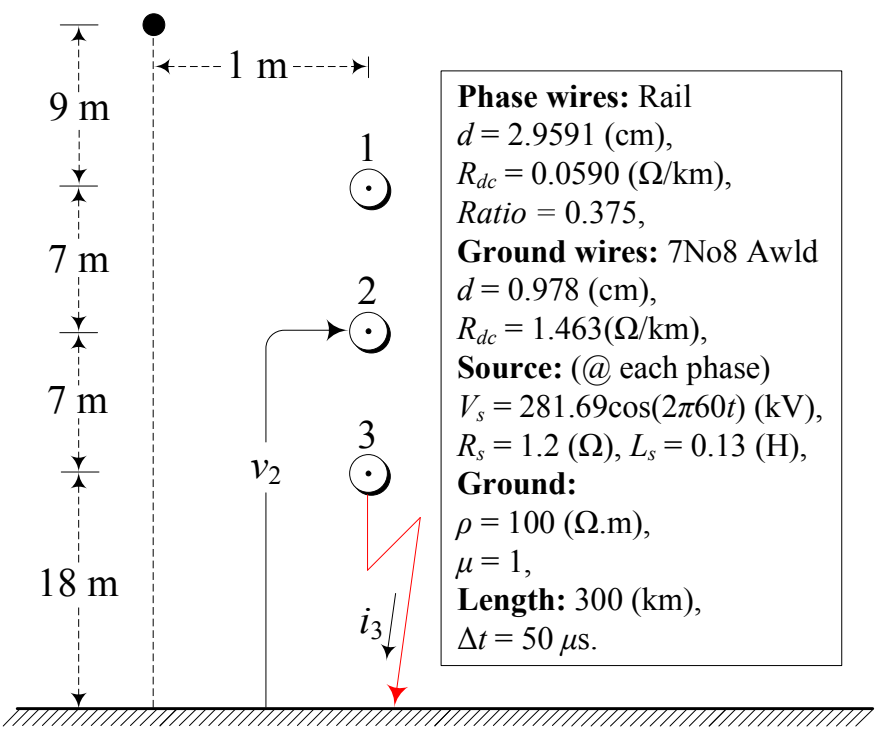

Figure 2.7: Three-phase single-circuit vertical transmission line.

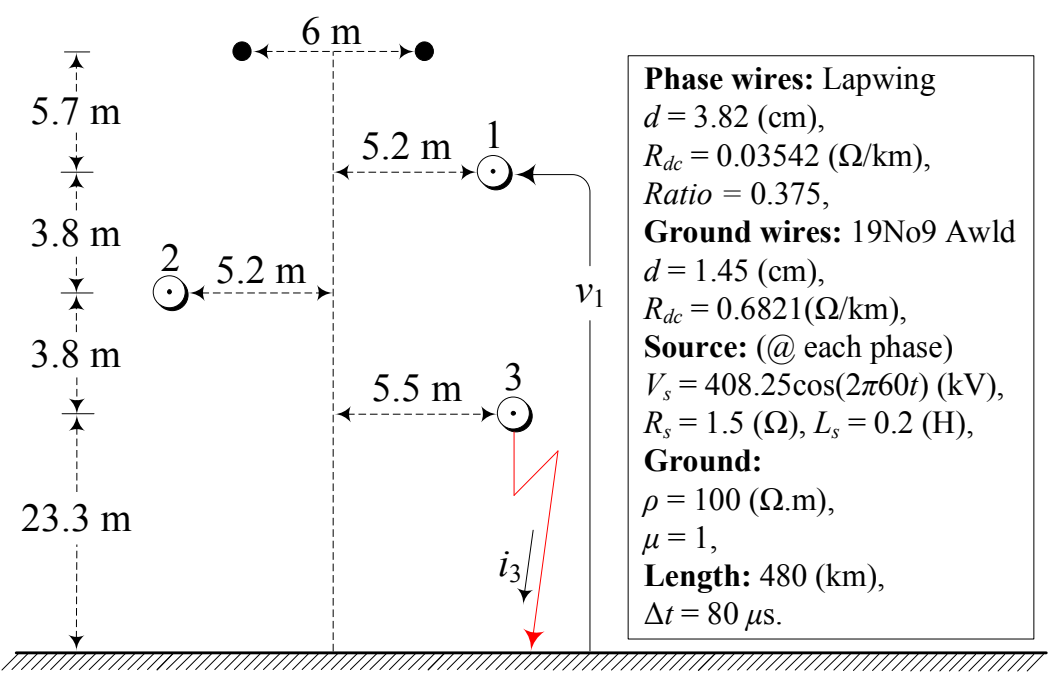

Figure 2.8: Three-phase single-circuit delta transmission line. 


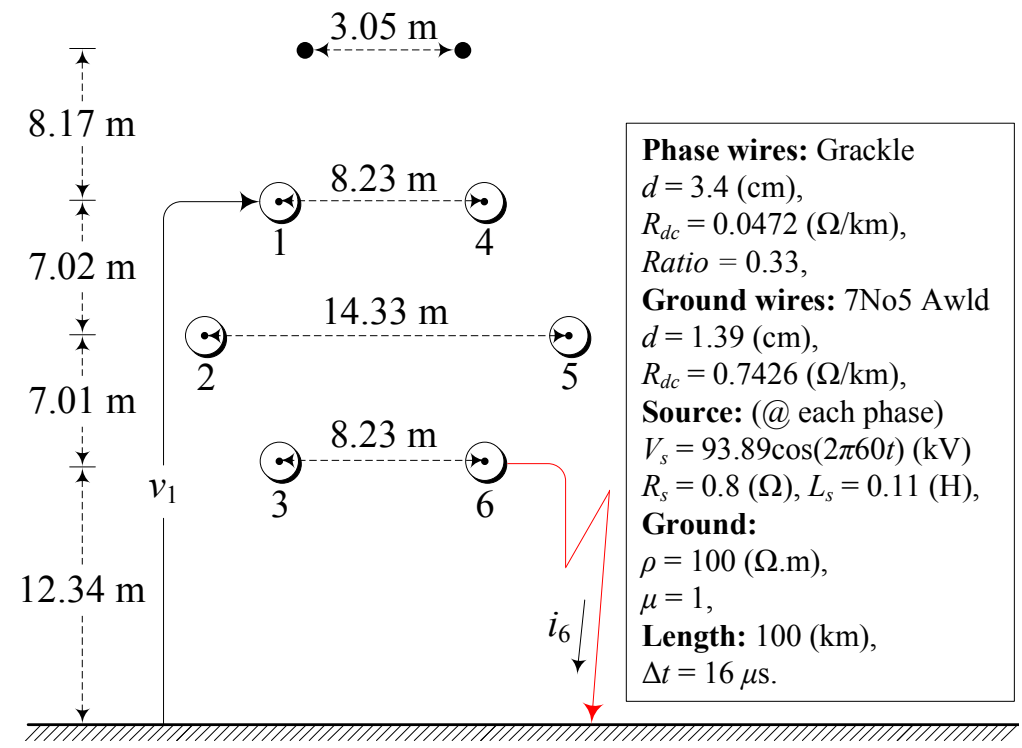

Figure 2.9: Three-phase double-circuit one-tower delta transmission line.

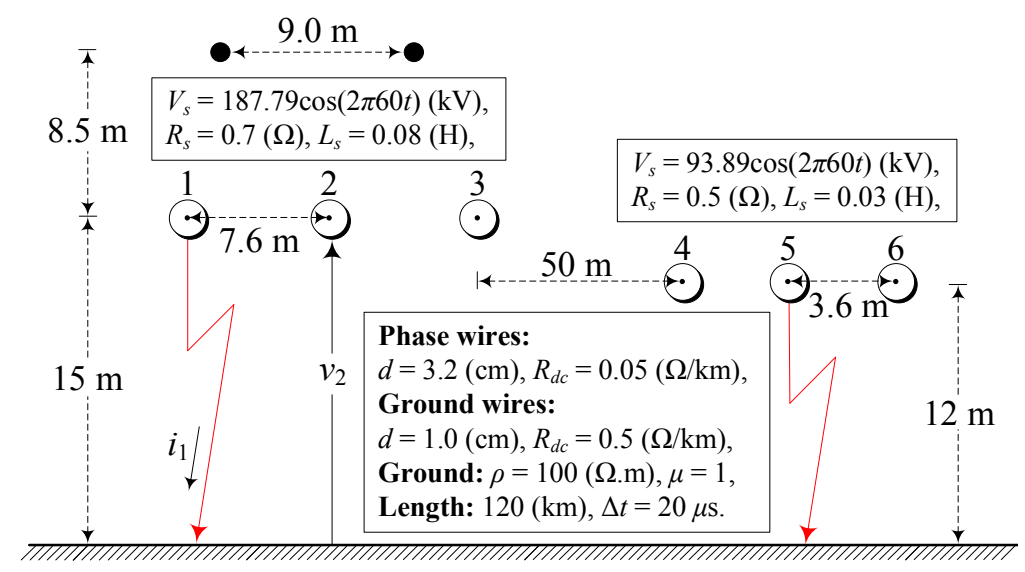

Figure 2.10: Three-phase double-circuit two-tower horizontal transmission line.

In the test cases, the transmission lines are connected to a balanced three-phase cosine source (Fig. 2.11) and the peak value of phase-a is applied at $t=0$. The equivalent source impedance corresponds to the impedance of the generator plus its step-up transformer. The conductors at the receiving-end of the line are either shorted or open (connected to the ground with a resistance of $10^{-6} \Omega$ or $10^{6} \Omega$, 
respectively). The simulations were run with the following conditions:

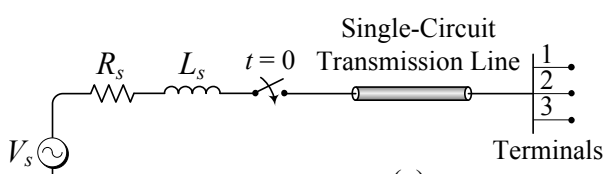

(a)

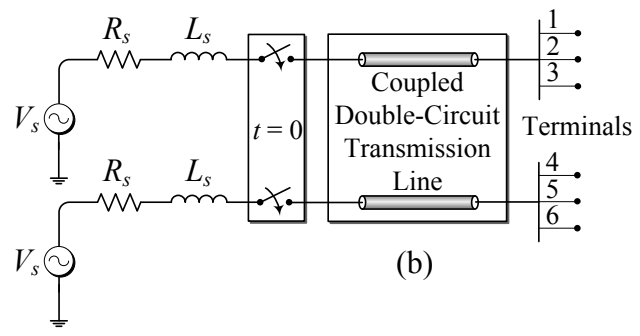

Figure 2.11: One-line diagram of the equivalent circuit for the tests. a) Single-circuit, b) Double-circuit.

Simulation tools: Microtran v3.25 for FDLINE, PSCAD v4.5.2 for ULM, and MATLAB for NLT. Even though particular software packages were used to run these tests, the cases are described with enough detail so that they can be run using other EMTP software implementations.

All methods: Shunt insulator losses $G_{i n s}$ was taken as $3 \times 10^{-8}(\mathrm{~S} / \mathrm{km})$ [2]. The simulations were run at the time steps $\Delta t$ 's indicated in the line configuration, Figs. 2.5 to 2.10. To guarantee the accuracy of the simulations, time steps are taken to be 20 times smaller than the travelling time $\tau$ of the line with the length $\ell$ at the speed of light $v_{c}=3 \times 10^{8} \mathrm{~m} / \mathrm{s}\left(\Delta t=\frac{1}{20} \tau=\frac{\ell}{20 v_{c}}\right)$.

Line models: For the curve-fitting, maximum number of poles was set to 35 over a frequency range from $10^{-2}$ to $10^{7} \mathrm{~Hz}$. The large frequency range is chosen to increase the accuracy of fitting by allowing the asymptotic function to extend asymptotically beyond the range at which it will be used, which for switching transients is about a few hundred $\mathrm{kHz}$ (for the calculation of line parameters, maximum frequency is chosen at $1 \mathrm{MHz}$ ). For FDLINE, $\mathbf{T}_{V}$ and $\mathbf{T}_{I}$ were calculated at $1 \mathrm{kHz}$.

NLT: Odd sampling, Error criterion $\left(\sigma_{3}\right)$ with $\varepsilon$ taken as $10^{-3}$, and Hanning window. Frequency window $f_{c}$ is $1 / \Delta t$ of the corresponding time steps. Number of samples equals $2^{20}$. 


\subsection{Comparison of Simulation Results}

The simulation results of the line energization tests, obtained with the NLT, FDLINE, and ULM solutions are shown in Figs. 2.12 to 2.17. Due to the closeness of the results presented in each plot, the differences are best viewed using the glass magnifier in a pdf viewer. The simulations are shown for a transient period $t=0$ to $t=50$ ms. Steady-state errors are demonstrated in Table 2.1. Due to space limitations, only the phases with higher errors are shown.

\subsubsection{Single-Phase Line}

The results for the single-phase line of Fig. 2.5 are shown in Fig. 2.12. These results show that, in the absence of transformation matrices needed in the multiphase cases, the BAF and VF curve-fitting algorithms used in FDLINE and ULM, respectively, can approximate the frequency dependence of the line functions quite accurately since the time domain solutions given by these line models perfectly match with the NLT solution which does not use these approximations.
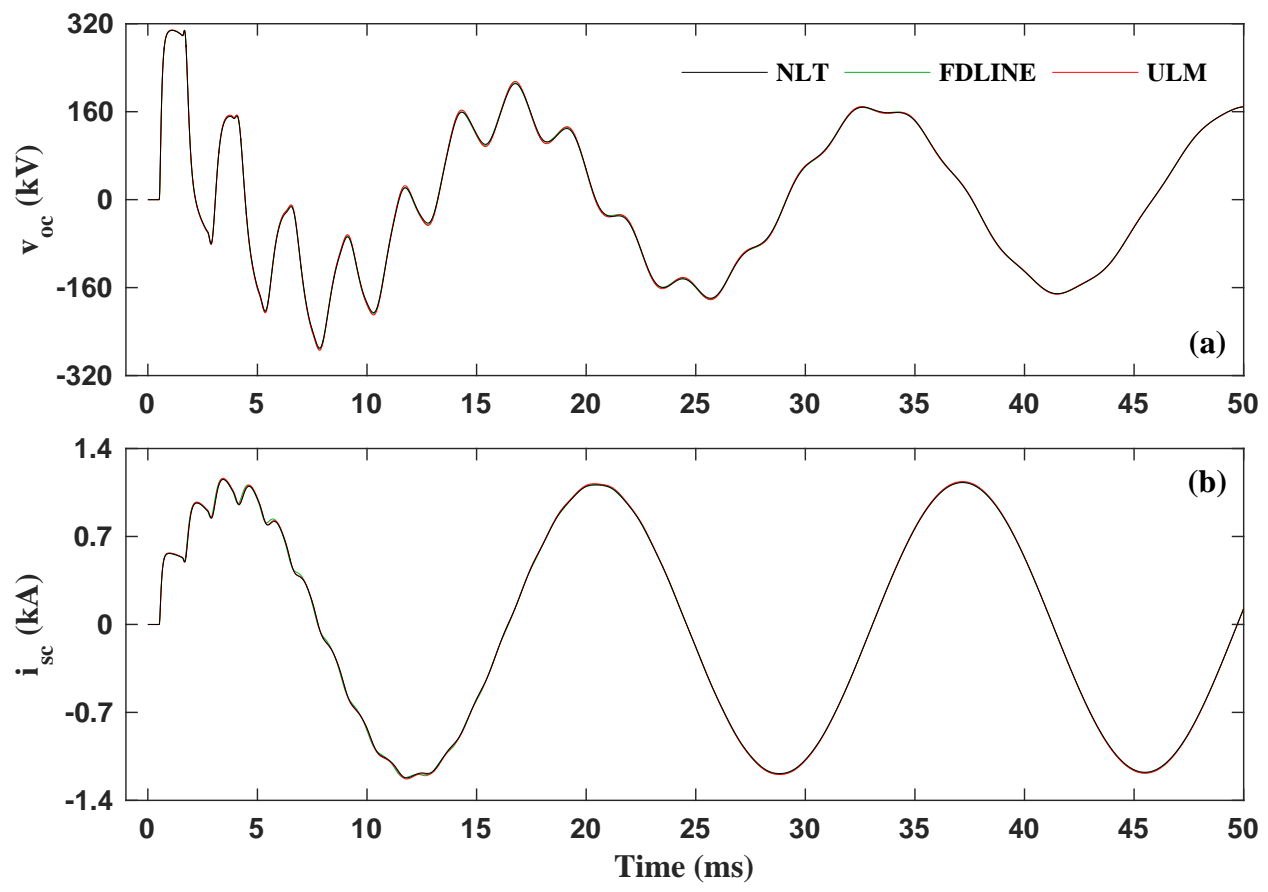

Figure 2.12: Simulation results for the single-phase line of Fig. 2.5. 


\subsubsection{Three-Phase Single-Circuit Lines}

The results for the single-circuit line tests are shown in Figs. 2.13 to 2.17. For the horizontal line of Fig. 2.6, the open-circuit voltages at the receiving-end of the line are shown in Fig. 2.13a. These results show that the solutions given by FDLINE and ULM are perfectly matched, and they follow the NLT solution very well (except in the jagged points of the curves in which the NLT deviates slightly). Also for the short-circuit currents, Fig. 2.13b shows that the results of FDLINE and ULM for the horizontal line are quite in agreement with the NLT solution.
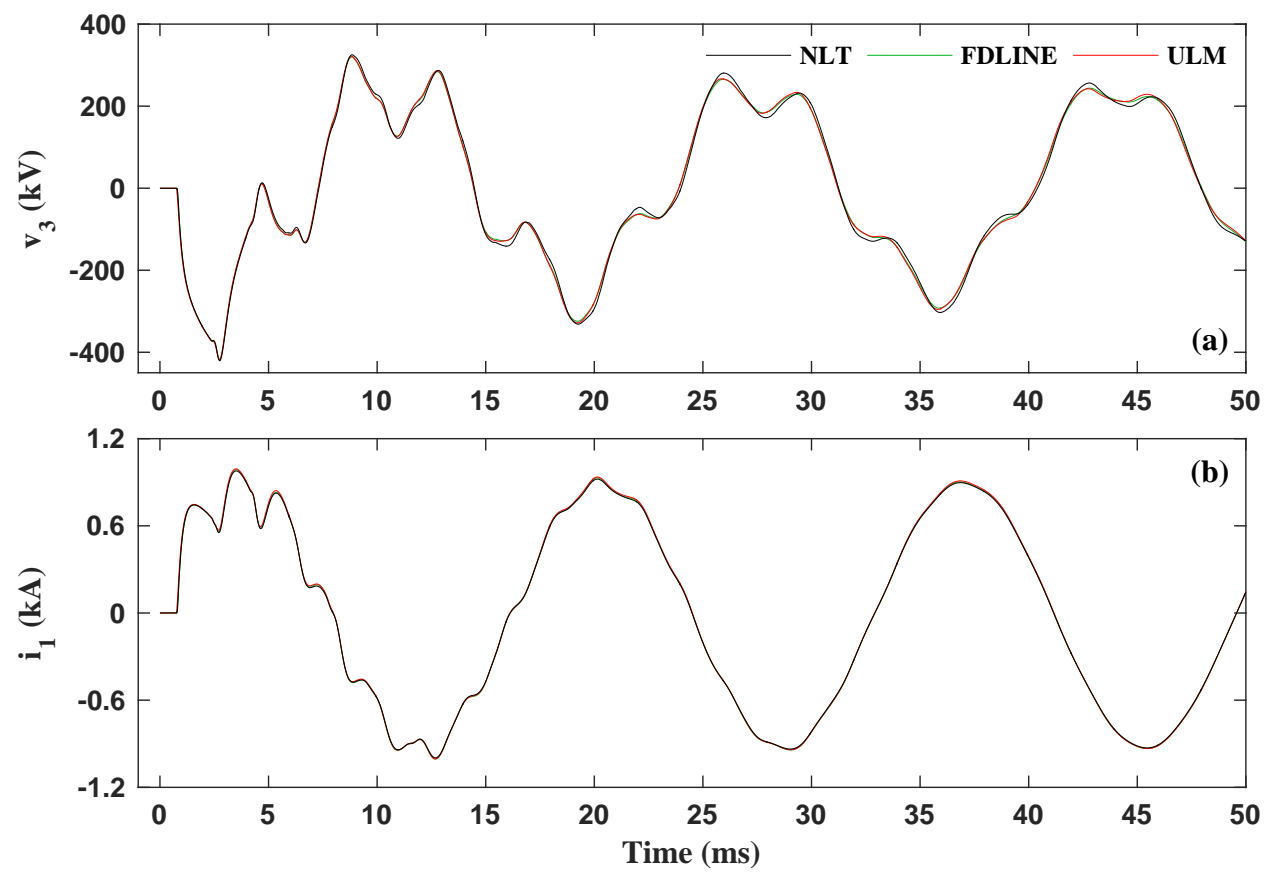

Figure 2.13: Simulation results for the horizontal line of Fig. 2.6.

For the vertical line of Fig. 2.7, the open-circuit voltages at the receiving-end of the line are shown in Fig. 2.14a. These results show that FDLINE follows the NLT solution closer than ULM. The deviation of the open-circuit voltages at the peak points and the slight phase shift drift of ULM can be more clearly observed. This phase shift is due to the lack of accuracy in ULM to determine the time delay which is caused by allowing non-minimum-phase shift fitting. For the short-circuit 
currents, Fig. 2.14b shows that ULM is closer to the NLT solution than FDLINE. The peak value of short-circuit current of FDLINE is about $5 \%$ below the peak value of the NLT and ULM solutions. The reason for this difference might be due to taking the transformation matrices at $1 \mathrm{kHz}$ in FDLINE which for this simulation is not a good choice. This issue will be analyzed in Section 3.8 .
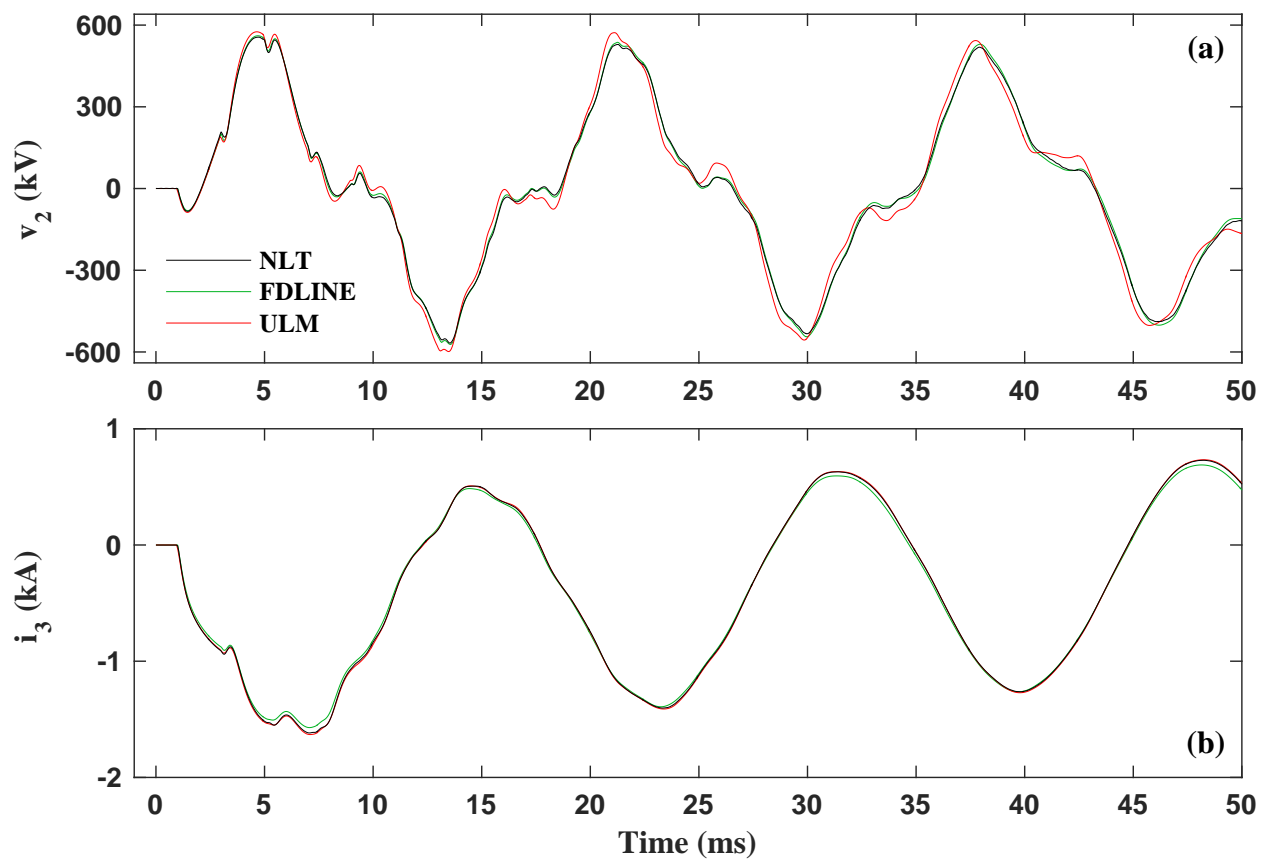

Figure 2.14: Simulation results for the vertical line of Fig. 2.7.

For the delta line of Fig. 2.8, the open-circuit voltages at the receiving-end of the line are shown in Fig. 2.15a. These results show that FDLINE and ULM follow the NLT solution well. However, there is no perfect match between the three solutions at the peak values of the few initial cycles. For the short-circuit currents, Fig. 2.15b shows that the short circuit currents of FDLINE and ULM match NLT solution very well.

\subsubsection{Three-Phase Double-Circuit Line in the Same Tower}

The FDLINE and ULM models performed very accurately for the single-phase line and the three-phase single-circuit lines. As expected, however, larger differences 

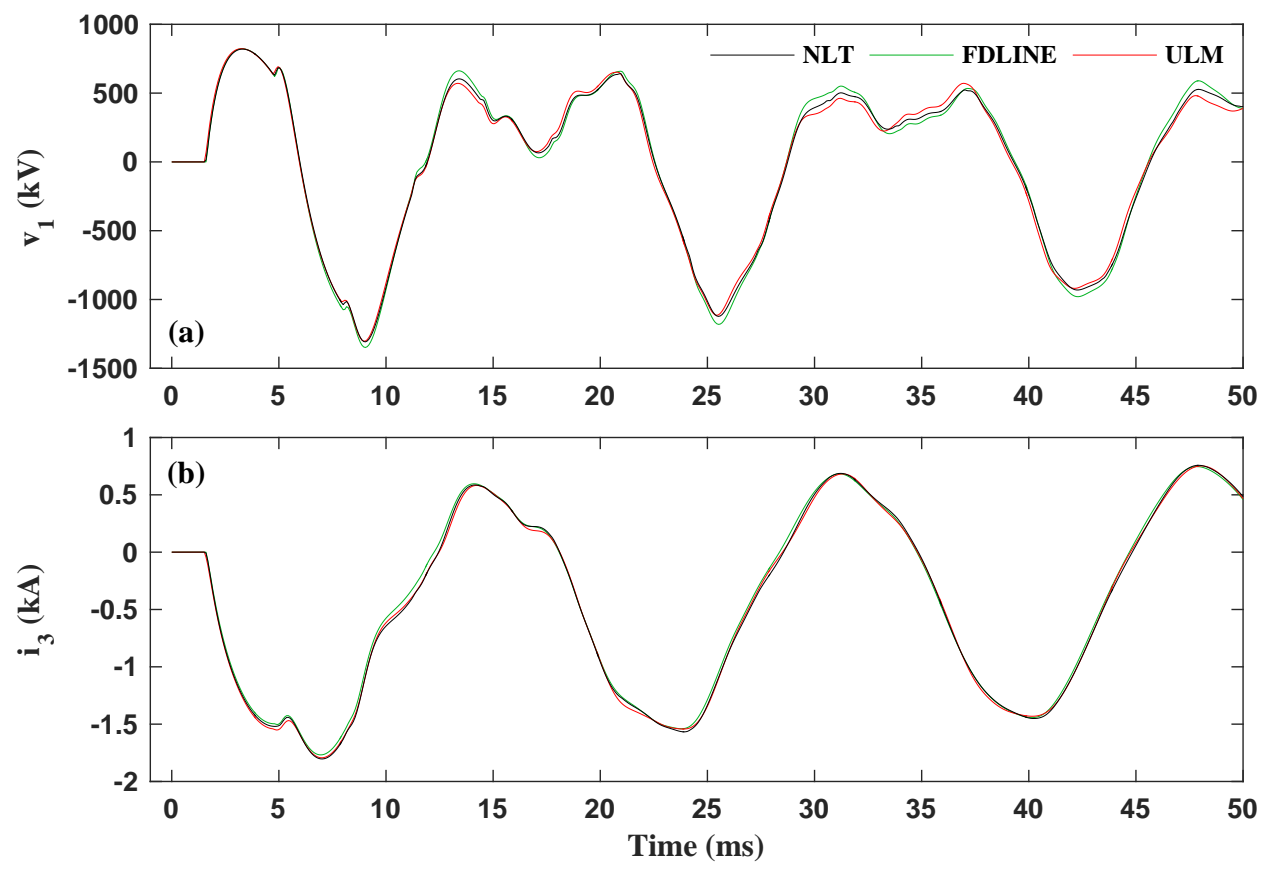

Figure 2.15: Simulation results for the delta line of Fig. 2.8.

between time-domain (FDLINE and ULM) and NLT simulations were observed for the double circuit lines. The reason is due to more coupling between the conductors in double circuit lines than the single circuit lines where the problem of frequency dependence of the transformation matrices for the line models and reaching the optimal setting for the NLT solution become more challenging. The results of these tests are shown in Figs. 2.16 to 2.17.

For the double circuit delta lines of Fig. 2.9, the open-circuit voltages at the receiving-end of the line are shown in Fig. 2.16a. As observed, the FDLINE and ULM solutions are very close to each other and follow the NLT solution quite well in the initial few cycles. However, the NLT solution deviates from the line models in the time span between 20 to $50 \mathrm{~ms}$. For the short-circuit currents, Fig. 2.16b shows that both FDLINE and ULM solutions match the NLT solution perfectly. 

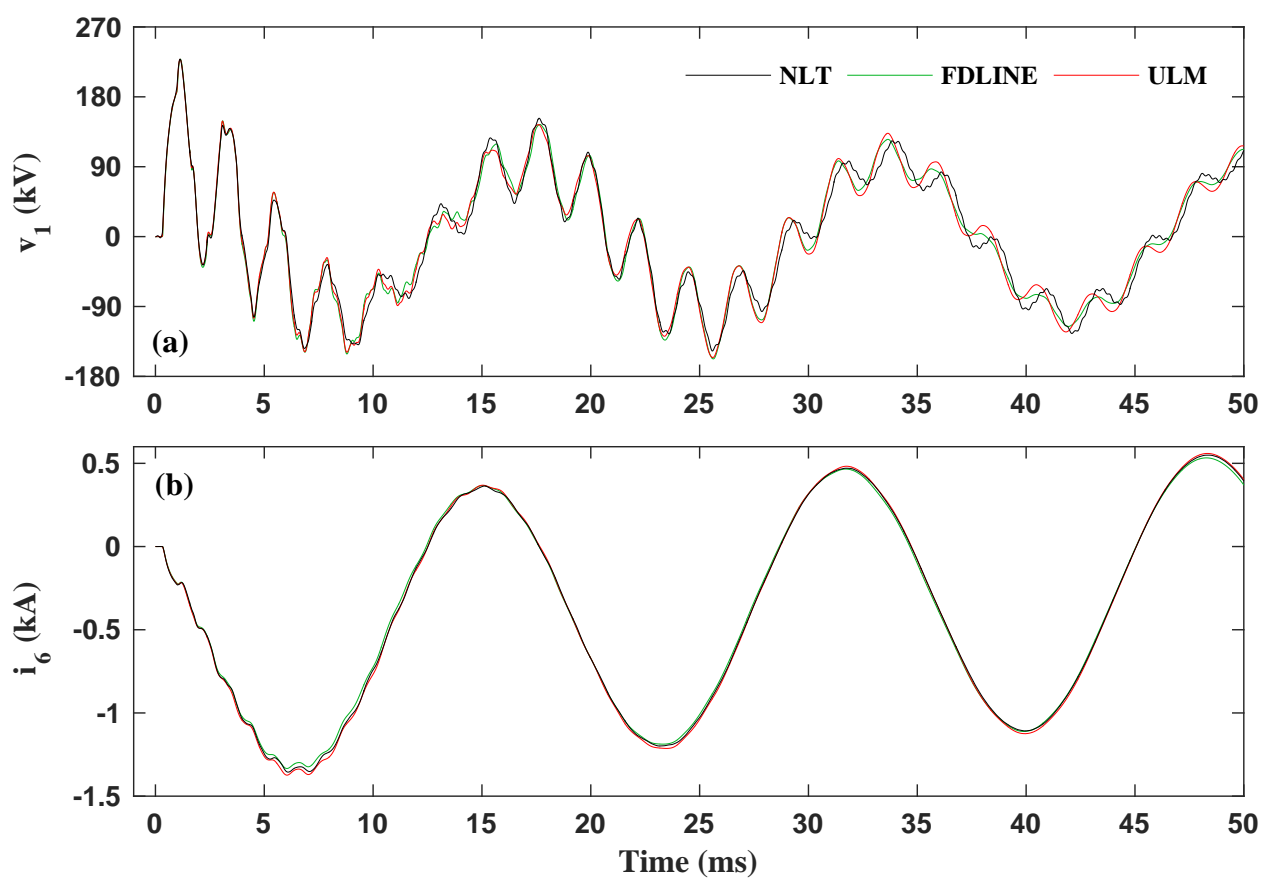

Figure 2.16: Simulation results for the double circuit delta line of Fig. 2.9.

\subsubsection{Three-Phase Double-Circuit Line in Separate Towers}

Figure 2.17 presents the results obtained for the two parallel horizontal lines of Fig. 2.10 mounted in two separate towers. For the open-circuit voltages, Fig. 2.17a shows that the solutions given by FDLINE and ULM are closer to the NLT solution than they were for the double circuit in the same tower case. This fact signifies that the choice of damping factor and window function for the NLT method were suitable for this test. As observed, FDLINE is slightly closer to the NLT solution than ULM, particularly at the peak points. For the short-circuit currents, Fig. 2.17b shows that the results are very similar for both FDLINE and ULM. However, the NLT solution presents a slight vertical shift with respect to the results obtained with line models. As opposed to the open-circuit voltage, for the calculation of shortcircuit current in two parallel horizontal lines, the NLT requires to use a different damping factor and/or windowing function so that it can match the result with the line models. 

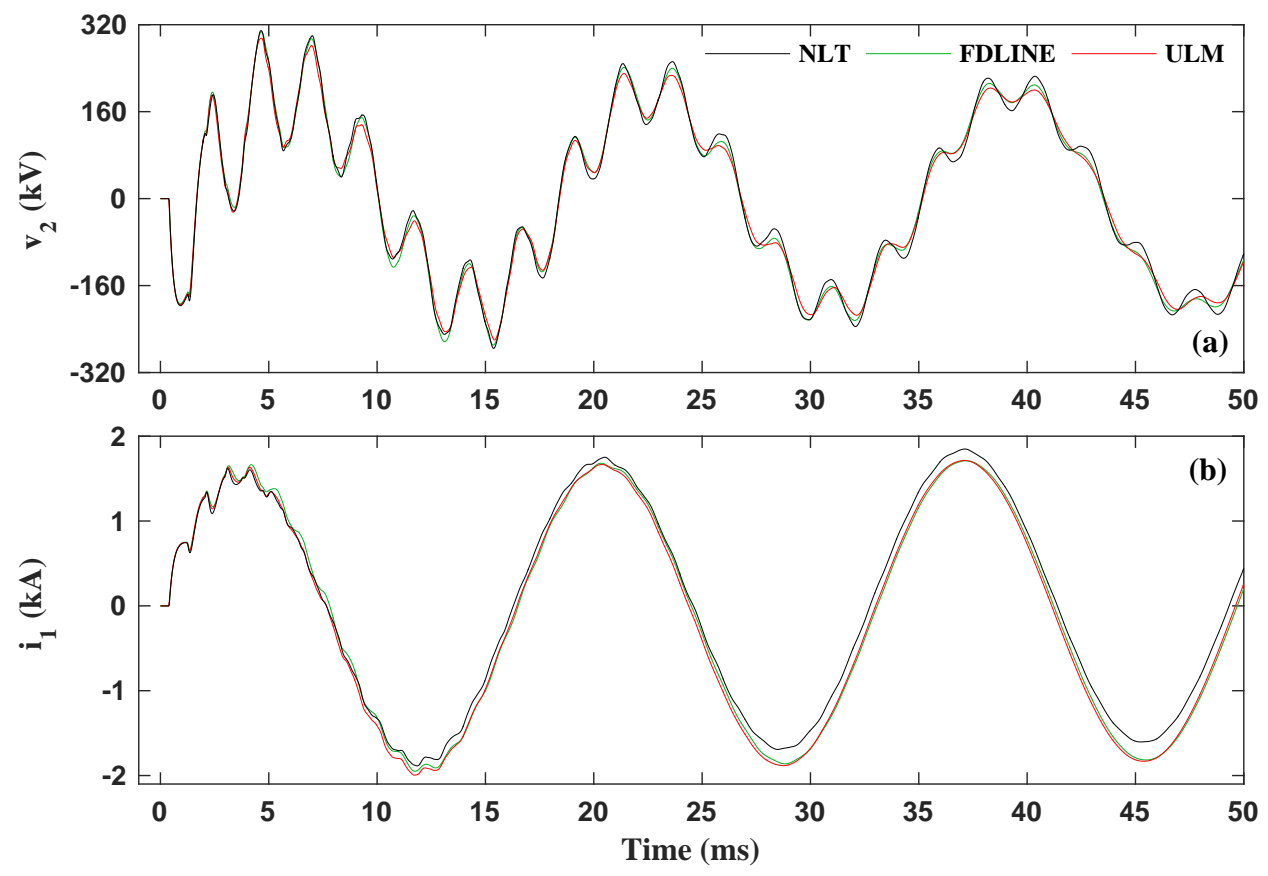

Figure 2.17: Simulation results for the two parallel horizontal line of Fig.

$$
2.10 \text {. }
$$

\subsubsection{Steady-state Solutions}

Table 2.1 shows the steady state solutions obtained with NLT, FDLINE, and ULM in the different test cases after the initial transient settles.

In this table, the maximum error for the steady state voltages was $1.16 \%$ for FDLINE and $1.25 \%$ for ULM. The maximum error for the steady state currents was $1.96 \%$ for FDLINE and $2.59 \%$ for ULM. Considering the complexity of frequency dependent line modelling, these are very good results for both FDLINE and ULM.

\subsection{General Observations}

Overall, simulation results showed that the FDLINE model gave similar results to the ULM. These results indicated that, contrary to traditional belief, a constant transformation matrix model like FDLINE is capable of representing multi-circuit asymmetrical line configurations. 
Table 2.1: Comparison of steady state values of different solutions.

\begin{tabular}{|l|c|c|cc|cc|}
\hline Test & $\mathrm{kV} / \mathrm{kA}$ & NLT & \multicolumn{2}{|c|}{ FDLINE } & \multicolumn{2}{c|}{ ULM } \\
\hline \hline \multirow{2}{*}{1} & $v_{o c}$ & 167.81 & 167.9 & $0.05 \%$ & 167.9 & $0.05 \%$ \\
\cline { 2 - 7 } & $i_{s c}$ & 1.155 & 1.159 & $0.36 \%$ & 1.163 & $0.69 \%$ \\
\hline \hline \multirow{2}{*}{2} & $v_{3}$ & 247.7 & 246.9 & $0.32 \%$ & 248.0 & $0.12 \%$ \\
\cline { 2 - 7 } & $i_{1}$ & 0.912 & 0.916 & $0.44 \%$ & 0.919 & $0.77 \%$ \\
\hline \hline \multirow{2}{*}{3} & $v_{2}$ & 397.5 & 396.9 & $0.15 \%$ & 399.4 & $0.48 \%$ \\
\cline { 2 - 7 } & $i_{3}$ & 0.970 & 0.951 & $1.95 \%$ & 0.976 & $0.62 \%$ \\
\hline \hline \multirow{3}{*}{4} & $v_{1}$ & 631.5 & 638.8 & $1.16 \%$ & 633.6 & $0.33 \%$ \\
\cline { 2 - 7 } & $i_{3}$ & 1.078 & 1.071 & $0.65 \%$ & 1.072 & $0.56 \%$ \\
\hline \hline \multirow{2}{*}{5} & $v_{1}$ & 100.6 & 101.1 & $0.50 \%$ & 100.5 & $0.10 \%$ \\
\cline { 2 - 7 } & $i_{6}$ & 0.809 & 0.803 & $0.74 \%$ & 0.820 & $1.36 \%$ \\
\hline \hline \multirow{2}{*}{6} & $v_{2}$ & 200.4 & 202.2 & $0.90 \%$ & 197.9 & $1.25 \%$ \\
\cline { 2 - 7 } & $i_{1}$ & 1.738 & 1.772 & $1.96 \%$ & 1.783 & $2.59 \%$ \\
\hline
\end{tabular}

The discrepancy between the time domain and frequency domain solutions emerged in the simulation of double circuit lines where the solutions given by FDLINE and ULM were very close. The reason for the deviation of NLT from the line models might be due to inappropriate choice of error criterion for the damping factor and Hanning for the windowing function used for the NLT method. However, this setting worked quite well for the single-phase line and three-phase singlecircuit lines.

Since the optimal set up for the NLT method is not easy to reach for non-expert users, as a result, in the next chapter we will investigate a simpler methodology in the frequency domain solutions which does not require the complicated set up as required for the NLT; while it provides very accurate results. 


\section{Chapter 3}

\section{Discrete Time Fourier Series Algorithm}

This chapter derives a Discrete Time Fourier Series (DTFS) formulation for frequency domain solutions of fast electromagnetic transients in power systems. The DTFS establishes a one-to-one mapping between $N_{s}$ samples in a finite time window and $N_{s}$ samples in a finite frequency window. The system response can be evaluated using nodal analysis to obtain $N_{s} / 2$ solutions in the frequency domain and this response is mapped back into $N_{s}$ solution points the time domain. The proposed methodology is based on the correct specification of the time window and frequency window widths. The DTFS is simpler to implement than classical NLT, which has been traditionally used for frequency domain solutions of power system transients. The DTFS can achieve similar levels of accuracy as the NLT without requiring user intervention to specify the value of the damping factor and filtering windows required in the NLT.

This chapter is organized as follows. First, the relationship between the discrete time domain and the discrete frequency domain is reviewed, then the steps to correctly set up a DTFS circuit solution are presented. Next, guidelines are provided to calculate a suitable time window and frequency window widths for the application of the DTFS. Simulation examples consisting of lumped $R, L, C$ parameters and frequency dependent transmission lines are introduced in preparation to compare the proposed DTFS with the conventional NLT and a reference EMTP 
solution. Finally, the proposed DTFS is used to assess the accuracy of the FDLINE and ULM line models under asymmetrical line energizations and fault conditions for a double circuit vertical line.

\subsection{Relationship Between Discrete Time Domain and Discrete Frequency Domain}

The relationship between discrete time domain and discrete frequency domain introduced in this section follows the point of view of [67] where a mapping is established between time and frequency points. For a time window of $N_{s}$ points there is a corresponding frequency window of exactly $N_{s}$ points.

Figure 3.1 illustrates a number of points in a time window of width $T_{c}$ and its corresponding frequency window of width $f_{c}$. As will be discussed in Section 3.2.1, the width of the time window will be chosen based on the time constants of the circuit ([123],[140]). The width of the frequency window will be chosen based on the maximum frequency that we want to simulate in the transient [28]. Once $T_{c}$ and $f_{c}$ have been chosen, the number of sample points (solution points) $N_{s}$ is determined by

$$
N_{s}=T_{c} f_{c}
$$

The time step size and the frequency step size are given by

$$
\Delta t=\frac{1}{f_{c}} \quad, \quad \Delta f=\frac{1}{T_{c}}
$$

Equations (3.1) and (3.2) indicate that to simulate a larger time window for a time step $\Delta t$ we will need more solution points. This is exactly the same as in the traditional EMTP criteria where one chooses the $\Delta t$ for a given maximum frequency and then solves the system at $N_{s}$ points $\left(N_{s}=t_{\max } / \Delta t\right)$ until $t_{\max }$ is reached.

It should be noted that because the Fourier series to represent a real signal needs to be complex conjugates (Fig. 3.1), the maximum frequency that can be captured in the solution is $f_{N y}$ (Nyquist frequency) which is one half of the sampling frequency,

$$
f_{N y}=\frac{1}{2 \Delta t}=\frac{f_{c}}{2}
$$




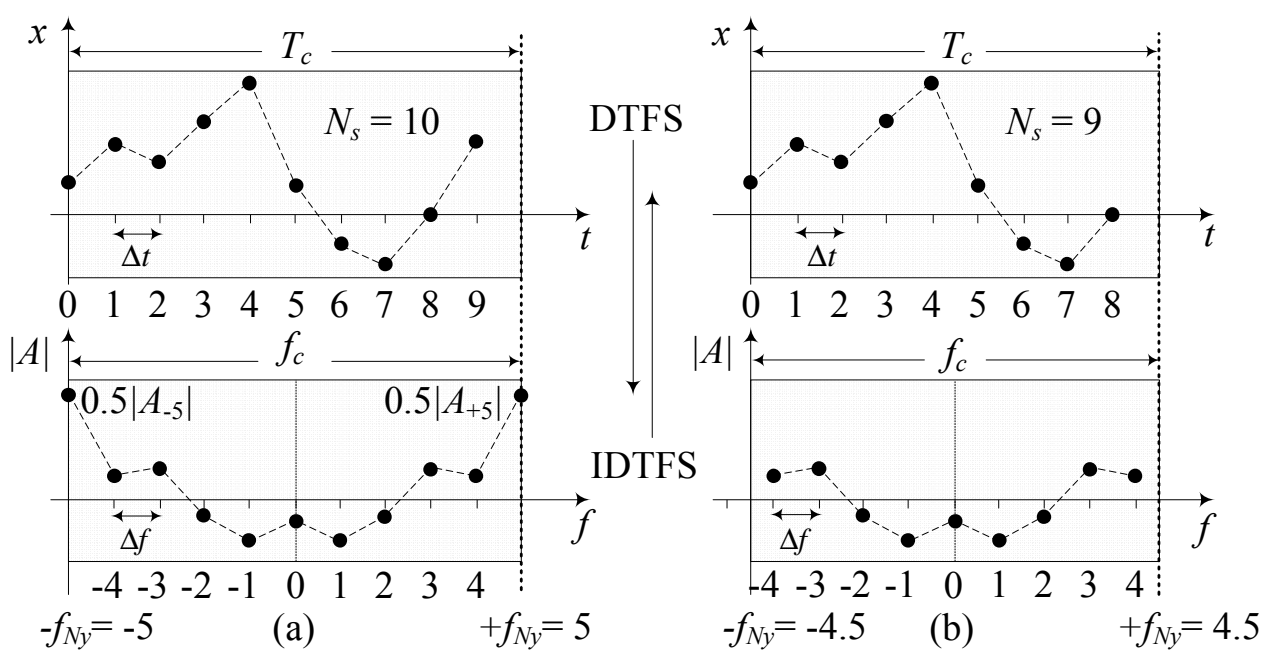

Figure 3.1: Correspondence of time domain and frequency domain for a discrete signal. a) $N_{s}=$ even. b) $N_{s}=$ odd.

This means that there will be only $N_{s} / 2$ distinct frequencies in the interval from zero to $+f_{N y}$. The rest of the frequency components from $-f_{N y}$ to zero are complex conjugate of the corresponding positive interval.

When simulating a physical system response, response samples are being produced as the source applies input samples at $t=n \Delta t$, for $n=0,1,2, \ldots, N_{s}-1$. After the response to all these samples has been produced, the simulation time window will contain a snapshot that is mapped into a frequency window with frequencies in increments $f=k \Delta f$, for $k=-\left(N_{s}-1\right) / 2, \ldots,-1,0,1, \ldots,\left(N_{s}-1\right) / 2$.

Notice in Fig. 3.1a, for $N_{s}=$ even that $\left(N_{s}-1\right) / 2$ is not an integer, then the closest next integer is used. In Fig. 3.1b, for $N_{s}=$ odd, the frequency component at $f_{N y}$ is split into two equal parts, one half on the negative side and the other half on the positive side. Even though the frequency window of Fig. 3.1a shows 11 points, there are only 10 distinct frequencies since the $-f_{N y}$ and $+f_{N y}$ points are $1 / 2$ of the same point and the coefficient at $f_{N y}$ is real (similarly, the coefficient at $f=0$ is also real). The frequency snapshot will provide frequency complex exponentials that can synthesize the values in the time snapshot. This synthesis is indicated in (3.4).

The width of the time window $T_{c}$ is determined by the desired simulation time 
and how long the system response lasts. Similarly to the case of the frequency window where only half of the window contains information, not all the time window contains information related to the solution of the transient. This is a crucial point in the solution proposed in this research.

The mapping between time and frequency in the DTFS is established as follows. A discrete-time signal $x[n]$, having an arbitrary shape and a finite number of equally spaced samples $N_{s}$, can be expressed as a sum of complex frequency components such that when evaluated at $t=n \Delta t$ we get the original $x[n]$ values,

$$
x[n]=\sum_{k=\left\lfloor-\frac{N_{s}-1}{2}\right\rfloor}^{\left\lceil\frac{N_{s}-1}{2}\right\rceil} A_{k} e^{j k \frac{2 \pi}{N_{s}} n}=\operatorname{IDTFS}\left\{A_{k}\right\}
$$

Equation (3.4) is the Inverse Discrete Time Fourier Transform (IDTFS) of the coefficients of the Fourier series. These coefficients are the spectral coefficients and are obtained from

$$
A_{k}=\frac{1}{N_{s}} \sum_{n=0}^{N_{s}-1} x[n] e^{-j k \frac{2 \pi}{N_{s}} n}=\operatorname{DTFS}\{x[n]\}
$$

Since the complex exponentials in the Fourier series are periodical functions, the synthesis of the time series is valid only for the original time span $T_{c}$.

\subsection{Power System Transient Solution Using the DTFS}

The impulse response of the system $h[n]$, which is the response of the system when a discrete-time impulse (a sample of value one) $i[0]=1$ is applied, is illustrated in Fig. 3.2a. If the sample of value one is applied at $n=1$ instead of $n=0$ (delayed impulse), the shape of the response will be the same but shifted by one, i.e., $h[n-1]$. For the next unit sample we will get $h[n-2]$, etc. All these subsequent responses will have the same shape as Fig. 3.2a but shifted to the right according to when the pulse is applied.

If the magnitude of the input samples is $i[n]$ instead of one, then the corresponding responses will be $i[0] h[n], i[1] h[n-1], i[2] h[n-2]$, etc. The total output by the time we reach point $N_{s}$ will be the sum of all responses to previous inputs plus the response to the current input, 


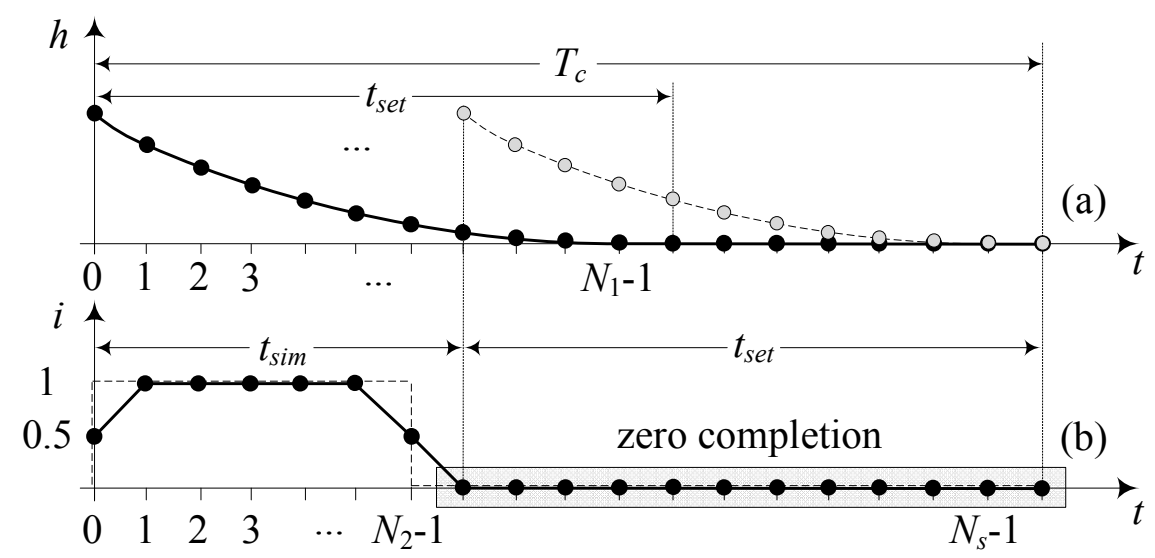

Figure 3.2: Time window selection. a) System impulse response $h[n]$, b) Current source $i[n]$ (Dashed line: unit step with discontinuity, Solid line: unit step with the averaging of points at the discontinuity).

$$
v[n]=i[n] * h[n]=\sum_{n=0}^{N_{s}} i[n] h\left[N_{s}-n\right]
$$

Equation (3.6) corresponds to the operation of convolution of the input function $i[n]$ and the system response $h[n]$ evaluated at $N_{s}$. This procedure will allow us to find the output $v[n]$ knowing the input $i[n]$ and the system response $h[n]$.

Suppose now that instead of obtaining the system response $h[n]$ in the time domain, our system is defined by a matrix of admittances $Y[k]$ that has different values at different frequencies $f=k \Delta f$. In this case, if we express the input source $i[n]$ in terms of its frequency components using (3.5) $I[k]=\operatorname{DTFS}\{i[n]\}$, we can calculate $V[k]$ by multiplying $Y^{-1}[k]$ by $I[k]$ using the property that convolution in time (3.6) is equivalent to multiplication in frequency. Once $V[k]$ has been calculated for each $k$, we can map the $V[k]$ sequence to its corresponding $v[n]$ sequence using (3.4).

For the process described to work, we need a time window of adequate width and a frequency window of adequate width so that the mapping between time domain and frequency domain can be unique and can include all time dynamics and all frequency components in the simulated system. 


\subsubsection{Defining the Time Window Width $\left(T_{c}\right)$}

Suppose we want to calculate the output $v[n]$ to some input $i[n]$ from $n=0$ to $n=N_{2}-1$. Assume the system response $h[n]$ (Fig. 3.2a) lasts for $N_{1}$ samples. Then the time window $T_{c}$ must be able to accommodate all the points of the source $\left(N_{2}\right)$ plus the length of the response $h[n]\left(N_{1}\right)$ to the last sample applied at $N_{2}-1$. This gives us a length of $N_{1}+N_{2}$ which must fit in the time window $T_{c}$. Calling the length of the source $t_{\text {sim }}$ and the length of the system impulse response $t_{\text {set }}$, we will have that $T_{c}=t_{\text {sim }}+t_{\text {set }}$. This is the minimum width for the response modes of the impulse response to die out within the time window. In Section 3.3.1, guidelines are provided to calculate the correct $t_{\text {set }}$.

When transferring sequences between time window and frequency window, all time sequences must have the same number of points in order to map the simulation $N_{s}$-point time window into the simulation $N_{s}$-point frequency window. Since the source sequence is only of length $N_{2}$ we need to complete this sequence with zeros ("zero completion") for the rest of the $T_{c}$ window so that the system response can settle without further source inputs. Since we stop the source at $t_{\text {sim }}$, the simulation will only be valid up to $t_{s i m}$. $T_{c}$ can be made larger than the minimum required for the purpose of increasing the frequency resolution (decreasing the spacing between frequencies) without changing $f_{c}$ and at the expense of calculating more simulation points $N_{s}$. Adding extra zeros to increase the frequency resolution is called "zero padding". This is not to be confused with completing the source function with zeros to allow the system response to settle ("zero completion").

\subsubsection{Defining the Frequency Window Width $\left(f_{c}\right)$}

For a given $T_{c}$ we can increase the time resolution by decreasing the size of $\Delta t$ at the expense of increasing the number of samples $N_{s}=T_{c} / \Delta t$. Decreasing $\Delta t$ will increase the Nyquist frequency $f_{N y}$ and therefore higher frequency components will be simulated. As opposed to the criticality of the correct choice of the time window $T_{c}$, to incorporate all the transient and obtain correct results, the choice of $\Delta t$ is determined by the maximum frequency we want to simulate in the transient. In discrete-time discrete-frequency analysis, if a transient contains frequencies up to $10 \mathrm{kHz}$ and we choose a $\Delta t$ for $f_{N y}=5 \mathrm{kHz}$, the system will be solved at discrete 
frequencies up to $5 \mathrm{kHz}$. If the actual transient were to have higher frequencies, our solution will be only an approximation of the correct solution.

In the case of using a continuous Laplace or Fourier transformation on a continuous source signal, higher source frequencies are captured. However, when solving the system for only a finite range of frequencies (of necessity because we can only solve for a finite number of points $N_{s}$ ), the time solution will present Gibbs oscillations due to the missing frequencies that we leave out of the solution.

Guidelines for the calculation of $f_{N y}$ will be presented in Section 3.3.2.

\subsubsection{System Solution Using the DTFS}

After identifying a suitable time window where the transient solution will be located, and a suitable frequency window for the frequencies of interest, we can transfer the source sequence from time to frequency using (3.5) and use nodal analysis to solve the system one frequency at a time $(f=k \Delta f)$ for $k=0,1,2, \ldots, N_{s} / 2$,

$$
\mathbf{V}[k]=\mathbf{Y}^{-1}[k] \mathbf{I}[k]=\mathbf{H}[k] \mathbf{I}[k]
$$

In (3.7), $\mathbf{V}$ is the calculated vector of output node voltages, $\mathbf{Y}$ is the matrix of admittances evaluated at each frequency ( $\mathbf{H}$ is the inverse of $\mathbf{Y}$ ), and $\mathbf{I}$ is component $k$ of the DTFS of the injected current sources in the window $T_{c}$. It is important to note that the DTFS is obtained for each individual current source of the matrix $\mathbf{i}[n]$. To avoid division by zero when taking the inverse of $\mathbf{Y}$, the DC frequency is taken as $10^{-4} \mathrm{~Hz}$.

After solving (3.7), the complementary part of the frequency spectrum of $V[k]$ (from $-f_{N y}$ to 0 ) is constructed by taking the complex conjugate of the positive half. For the NLT solution, this property only exists when regular sampling is considered (it does not apply to odd sampling). Finally, the time domain voltage at any node of interest is obtained by the synthesis of the time domain function from its frequency components using the IDTFS of (3.4). After evaluating the IDTFS to obtain $v[n]$ in the time window $T_{c}$, only the part of $v[n]$ from zero to $t_{\text {sim }}$ represents the output results. The rest of the time window corresponds to the de-energization response and are "auxiliary points" that should be discarded.

A source of errors when discretizing the input sources is related to disconti- 
nuities in these sources. Consider, for example, a unit step function applied to a system (Fig. 3.2b, dashed line). Because the coefficients in the spectral synthesis of the DTFS are continuous periodical functions, they cannot match discontinuities well. In addition, the DTFS formula uses a rectangular (forward Euler) approximation of the "area" under the discrete time points, which results in a less accurate synthesis than using, for example, a trapezoidal approximation to this area. Fig. 3.16 in Section 3.5.2 illustrates the consequences of these problems. A considerable improvement to this situation is to use the average of the values at the discontinuities, as indicated in Fig. 3.2b (solid line). This makes the calculated area equal to using the trapezoidal rule for the area under the discrete time points.

\subsection{Guidelines to Specify $T_{c}$ and $f_{c}$}

In this section, guidelines are provided to calculate $t_{s e t}$ and $f_{N y}$ which are needed to calculate the width of the time and frequency windows, $T_{c}$ and $f_{c}$. The first step is to extract the poles from the system's transfer function. The $n$ poles for a stable system of order $n$ are:

$$
p_{i}=\alpha_{i} \pm j \beta_{i} \quad, \quad i=1,2, \ldots, n
$$

where, $\alpha$ and $\beta$ correspond to the real part and the imaginary part of the system pole in this chapter.

\subsubsection{Calculation of the Time Window Width $\left(T_{c}\right)$}

As discussed earlier, the natural system responses must fit within the time window, and for this to happen $T_{c}$ minimum should be $t_{\text {sim }}+t_{\text {set }} . t_{\text {sim }}$ is the simulation time of interest specified by user and $t_{\text {set }}$ is the time interval needed by the system response to decay to zero. To ensure that the transient is completely damped within $t_{\text {set }}$, we recommend to take this time as seven times the time constant of the slowest mode of the system $\tau_{m}\left(t_{\text {set }}=7 \tau_{m}\right)$. This choice will be more justified by performing a sensitivity analysis in Section 3.6. The negative of the real part of the poles is the inverse of the time constants. The largest time constant of the system $\tau_{m}$ is calculated as

$$
\tau_{m}=\frac{1}{\min \left(-\alpha_{i}\right)}
$$


An approach to calculate $\tau_{m}$ for a system that includes frequency dependent transmission lines will be presented in Section 3.4.1.

\subsubsection{Calculation of the Frequency Window Width $\left(f_{c}\right)$}

For an accurate representation of the system, the width of the frequency window $f_{c}$ must be such that the system resonances fall within $f_{N y}=f_{c} / 2$. As a guideline, $f_{N y}$ should be at least as large as the maximum of the following bandwidths:

$B_{r}$ : bandwidth of the real part of the system poles,

$B_{i}$ : bandwidth of the imaginary part of the system poles,

$B_{\ell}$ : bandwidth of the transmission line,

$B_{s}$ : bandwidth of the source,

$B_{w}$ : bandwidth of the switching for power electronic devices,

$B_{e}$ : bandwidth of the estimation algorithm.

The real part of the system poles corresponds to decaying exponentials in the time domain. The decay of the exponentials was taken into account for the width of the time window in (3.9). However, it also provides an index for the frequency bandwidth,

$$
B_{r}=\max \left(-\alpha_{i}\right) P P C
$$

In (3.10), $B_{r}$ is augmented by a factor Points Per Cycle (PPC) to provide a suitable resolution. We recommend a PPC of 10 .

For some systems, the real part amplitude of some of the poles $(\alpha)$ might be much larger than that of other poles. Very large $\alpha$ means that the associated transient decays very fast and for the time span of the simulation their contribution may be negligible and ignoring them will prevent unnecessarily high values of $f_{c}$. It is recommended that values of $\alpha$ larger than $5 \times 10^{4}$ do not be taken into account to limit the frequency window to $1 \mathrm{MHz}$ and the corresponding $\Delta t$ to $1 \mu \mathrm{s}$.

The bandwidth corresponding to the imaginary part of the system poles are given by

$$
B_{i}=\frac{\max \left(\left|\beta_{i}\right|\right)}{2 \pi} P P C
$$


For a system that includes transmission line, $B_{r}$ and $B_{i}$ are calculated excluding the line from the circuit. The bandwidth for a transmission line of length $\ell$, using the speed of light $v_{c}=3 \times 10^{8} \mathrm{~m} / \mathrm{s}$ is calculated as

$$
B_{\ell}=\frac{v_{c}}{\ell} P P C
$$

Common sources in power systems are: sinusoidal, decaying exponential, and step. For a sinusoidal source with frequency $f_{0}, B_{s}$ is calculated as

$$
B_{s}=f_{0} P P C
$$

The bandwidth for the decaying exponential source is calculated using (3.10). The bandwidth for a step function of duration $t_{\text {sim }}$ that contains $99 \%$ of the energy is calculated from Parseval's theorem [99],

$$
B_{s}=\frac{11}{t_{\text {sim }}}
$$

For a system with power electronic devices such as inverters, $B_{w}$ corresponds to the frequency of switching of power electronic switches.

The estimation algorithms are usually used for power quality studies to extract the amplitude and phase angle of different harmonics of a single [115] [117] [3]. The bandwidth associated with these algorithms are taken as the highest order of signal's harmonics.

Since the DTFS provides a one-to-one mapping between the chosen time and frequency windows, frequencies outside the specified frequency width do not exist. As a result, no frequency filtering windows are needed when using the DTFS, while they are normally needed when using the NLT to "chop" frequencies outside this window. Also since the entire transient response of the system fits inside the chosen time window, the proposed DTFS method does not require, like the NLT, the use of a damping factor $\sigma$ in the system solution.

\subsection{Case Studies}

The test cases in this chapter include single-phase circuits and MTL-circuits. 


\subsubsection{Single-phase Circuits}

A $300 \mathrm{~km}$ single-phase transmission line is considered as a test case, as shown in Fig. 3.3. A source is applied to the sending-end while the receiving-end is shorted through a resistance of $1 \Omega$. The conductor type is Rail and is placed 18 meters above lossy ground with the resistivity of $100 \Omega . \mathrm{m}$ [116].

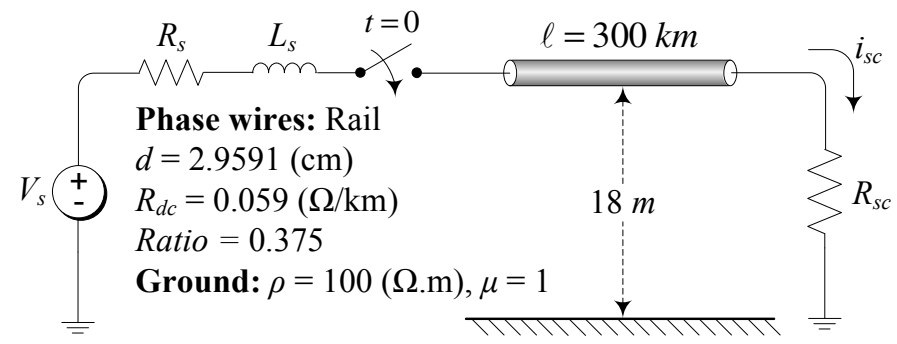

Figure 3.3: Example single-phase overhead transmission line.

Fig. 3.4 shows three line models that can be used to represent the transmission line of Fig. 3.3 in the frequency domain. The test circuits will consist of a voltage source, a source impedance $\left(R_{S}\right.$ and $\left.L_{S}\right)$, one of the line models of Fig. 3.4, and a terminating short circuit resistance $\left(R_{s c}\right)$. These circuits will be called: a) exact- $\pi$ circuit, b) nominal- $\pi$ circuit, and c) RL circuit.

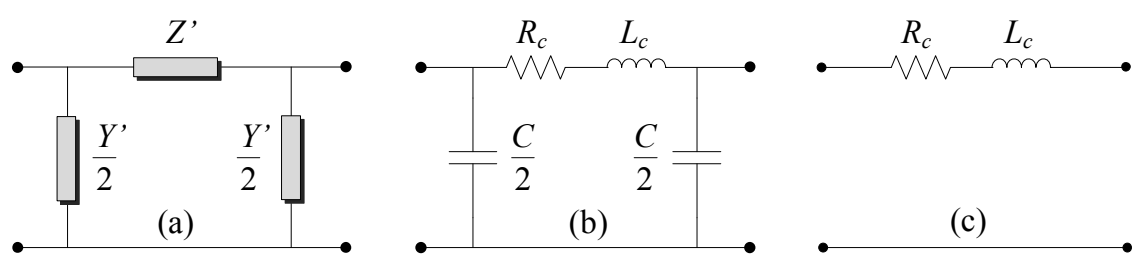

Figure 3.4: Single-phase line models. a) exact- $\pi, b)$ nominal- $\pi, c)$ RL.

The purpose for introducing the circuits with lumped elements (Figs. 3.4b and $3.4 c)$ is for preliminary validation of the DTFS method with the reference analytical formulas derived for these circuits.

The line is energized at $t=0$ via two types of voltage sources: a) a step voltage with $V_{m}=1 \mathrm{kV}$ for all three cases, and b) a cosine waveform with $V_{r m s}=200 \mathrm{kV}$ and $60 \mathrm{~Hz}$ for the exact- $\pi$ circuit. The equivalent source resistance and inductance 
include the generator and step-up transformer and are taken as $1.2 \Omega$ and $0.13 \mathrm{H}$, respectively.

The exact- $\pi$ model of Fig. 3.4a is calculated at discrete frequencies $(k \Delta f$ for $k=0,1,2, \ldots, N_{s} / 2$ ) based on the procedure given in Section 2.2.3. The series impedance and shunt admittances of the exact- $\pi$ circuit are obtained using the traditional calculation for the per unit length line parameters presented in Section 2.2.1. The shunt admittance $G_{\text {ins }}$ is considered as $2 \times 10^{-9} \mathrm{~S} / \mathrm{km}$ [39].

Nominal- $\pi$ model (Fig. 3.4b) is a simplified version of the exact- $\pi$ model for short lines and low frequencies. Under these conditions $G_{i n s}$ is neglected, $k_{z}$ and $k_{y}$

are assumed to be one, and $Z$ and $Y$ are calculated at the power frequency. For the example of Fig. 3.3, the lumped $R_{c}, L_{c}$, and $C$ are calculated at $60 \mathrm{~Hz}$ as $35.4 \Omega$, $0.68 \mathrm{H}, 2.14 \mu \mathrm{F}$, respectively.

The use of the nominal- $\pi$ model is introduced in the criteria to calculate the slowest time constant of the exact- $\pi$ model. The time constants of the exact- $\pi$ model are mainly associated with the inductances and capacitances. Since inductances decrease with frequency and capacitances are assumed constant under TEM assumption, and the correction factors at very low frequencies are one, the slowest time constant of the exact- $\pi$ model can be given by the nominal- $\pi$ model at very low frequencies.

The nominal- $\pi$ model can be further simplified into the RL model of Fig. 3.4c for short lines by removing the shunt capacitances.

The outputs are the short circuit current $i_{s c}$ for all the three cases and the branch voltage $v_{L}$ across the $L_{c}$ in Fig. 3.4c. For comparisons, analytical formulas are derived for the output of the RL circuit and the nominal- $\pi$ circuit as shown in Table 3.1.

\subsubsection{MTL-Circuits}

The test case considered in this section is a double circuit vertical line with the geometrical positions shown in Fig. 3.5 [34], which corresponds to the structure that resulted in higher errors in [116].

Modal lumped elements $\left(R_{c}, L_{c}, C\right)$ of the corresponding nominal- $\pi$ model of transmission line of Fig. 3.5 are given in Table 3.2. These parameters are calculated 
Table 3.1: Analytical formulas for the RL and nominal- $\pi$ circuits.

\begin{tabular}{|c|c|}
\hline \multicolumn{2}{|c|}{$\mathrm{RL}$ circuit } \\
\hline$i_{s c}(t)=\frac{V_{m}}{R_{e q}}\left(1-e^{p t}\right) \quad, \quad v_{L}(t)=\frac{V_{m} L_{c}}{L_{e q}} e^{p t}$ \\
\hline$R_{e q}=R_{s}+R_{c}+R_{s c}, L_{e q}=L_{s}+L_{c}, p=\frac{-R_{e q}}{L_{e q}}=-46.42$ \\
\hline \multicolumn{2}{|c|}{ Nominal- $\pi$ circuit } \\
\hline \multicolumn{2}{|c|}{$i_{s c}(t)=-V_{m} \sum_{i=1}^{4} \frac{k_{i}}{p_{i}}\left(1-e^{p_{i} t}\right)$} \\
\hline$k_{i}$ & $p_{i}$ \\
\hline$-1.2105 \times 10^{-5}$ & $-9.3458 \times 10^{5}$ \\
$-0.61723-j 0.0061354$ & $-8.1695+j 2926.3$ \\
$-0.61723+j 0.0061354$ & $-8.1695-j 2926.3$ \\
\hline
\end{tabular}

at $0.01 \mathrm{~Hz}$ which are further used to calculate the slowest time constant of the coupled exact- $\pi$ model.

Table 3.2: Modal lumped elements of the nominal- $\pi$ circuit for the double circuit vertical line of Fig. 3.5 calculated at $10^{-4} \mathrm{~Hz}$.

\begin{tabular}{c|cccccc}
\hline Modes & 1 & 2 & 3 & 4 & 5 & 6 \\
\hline$R_{c}(\Omega)$ & 17.7 & 17.7 & 17.7 & 17.7 & 17.7 & 17.7 \\
$L_{c}(\mathrm{H})$ & 4.3784 & 0.4925 & 0.4465 & 0.3631 & 0.3893 & 0.3882 \\
$C(\mu \mathrm{F})$ & 2.0747 & 4.5431 & 4.6674 & 5.7464 & 5.3475 & 5.5479 \\
\hline
\end{tabular}

The double circuit vertical line of Fig. 3.5 is energized at $t=0$ for the following tests: unbalanced faults, induced voltage, and step response. Because of space considerations, outputs are considered as the voltages and currents with highest errors. The simulations for the short circuit currents gave much smaller errors than 


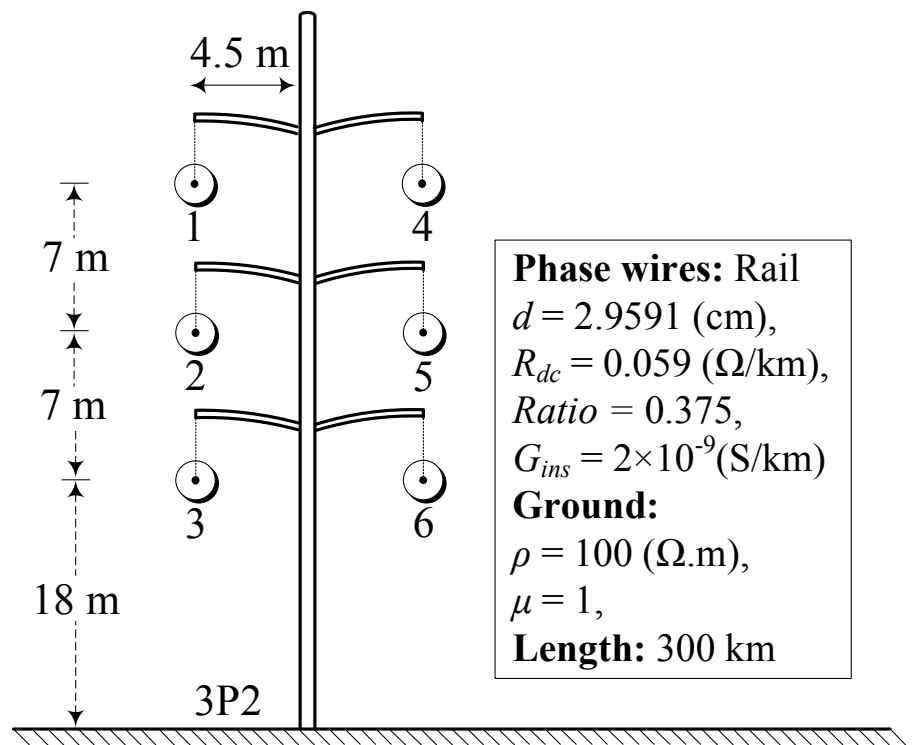

Figure 3.5: Double circuit vertical transmission line.

for the voltages.

\section{Unbalanced faults test:}

The equivalent circuit for this test is shown in Fig. 3.6. At the sending-end, each circuit is connected to a balanced three-phase cosine source and the peak value of phase-a is applied at $t=0$. The conductors $1,3,4$, and 5 at the receiving-end of the line are open (connected to the ground with a resistance of $10^{6} \Omega$ ), whereas conductors 2 and 6 are shorted with a resistance of $1 \Omega$. The outputs are the voltage at open conductor 3 and current at the shorted conductor 6 .

\section{Induced voltage test:}

Figure 3.7 illustrates the system diagram for the induced voltage test. This test can be performed for the maintenance of individual three-phase circuits. In this example, the bottom circuit is grounded at both sending-end and receiving-end with $10 \Omega$ resistances. The top circuit is energized with a three-phase balanced cosine voltage at sending-end with open conductors 1 and 3 and shorted conductor 2 with a $1 \Omega$ resistance at the receiving-end. The outputs are the current at shorted conductor 2 and the voltage at the terminal of conductor 6 . 


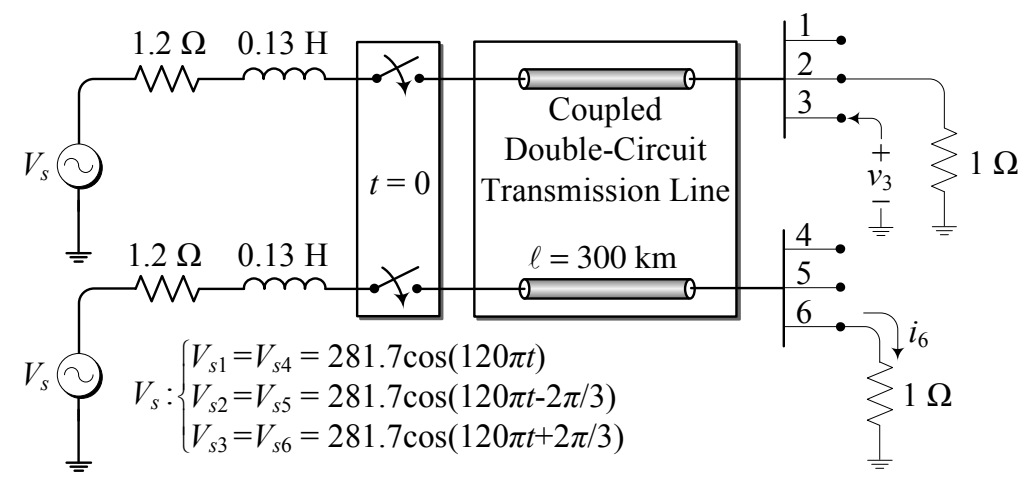

Figure 3.6: System diagram for the unbalanced faults test.

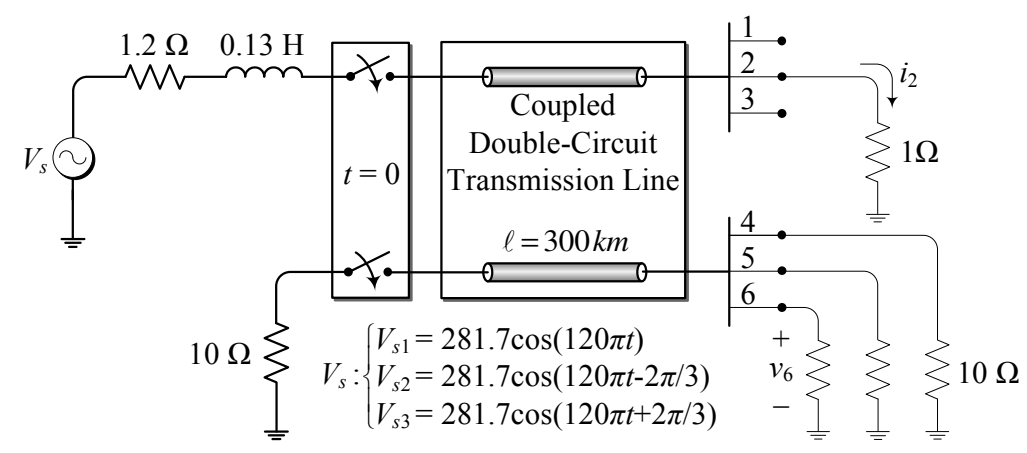

Figure 3.7: System diagram for the induced voltage test.

\section{Step response test:}

The equivalent circuit for this experiment is shown in Fig. 3.8. In this test, conductor 1 is energized with a step voltage with the amplitude of $1 \mathrm{kV}$ at sendingend, and is kept open at the receiving-end. The rest of the conductors are shorted with a $10 \Omega$ resistance at both sending-end and receiving-end. The voltage at open conductor 1 and the voltage at the shorted conductor 4 are considered as outputs in this test.

For simplicity, the test systems of Figs. 3.6 to 3.8 are called "MTL-circuits".

\subsection{Simulation Results}

In this section, the simulation results of the proposed DTFS method are compared with the NLT method, a reference EMTP solution run at a very small $\Delta t=1 \mu \mathrm{s}$, 


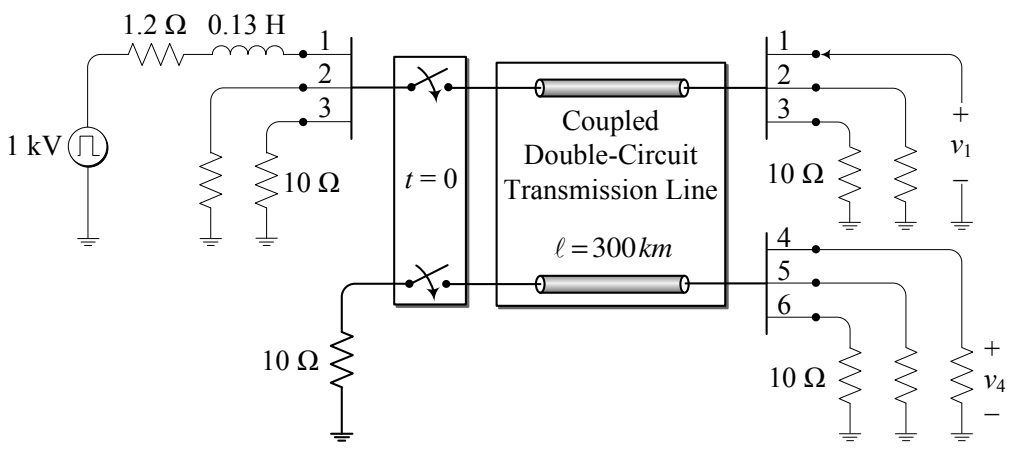

Figure 3.8: System diagram for the step response test.

and the analytical formulas for the nominal- $\pi$ and the RL circuits. The analytical formula is used to assess the accuracy of the DTFS and NLT in a form of an absolute error. For the exact- $\pi$ circuit and MTL-circuits, the solutions given by the DTFS and NLT are benchmarked with two EMTP line models: the FDLINE model using Microtran v3.25 and the ULM using PSCAD v4.5.2. In the curve-fitting process of FDLINE and ULM, the maximum number of poles is set to 35 , the frequency range considered is from $10^{-2}$ to $10^{7} \mathrm{~Hz}$ (as discussed in Section 2.3, the maximum range for frequency is chosen at $100 \mathrm{MHz}$ to increase the accuracy of the curve-fitting). For FDLINE, the transformation matrices are taken at $1 \mathrm{kHz}$ (for MTL-circuits). The NLT method used for these comparisons follows the regular sampling formulation given in [75]. For benchmarking purposes, the same time and frequency windows proposed in Section 3.3 for the DTFS are also used for the NLT. The damping factors used for the NLT are: Wilcox criteria $\sigma_{1}$, Wedepohl criteria $\sigma_{2}$, and error criteria $\sigma_{3}$ with $\varepsilon$ taken as $10^{-6}$. The windowing functions for the NLT solution are considered as Lanczos, Hanning, and Tukey with $2 Q / N=0.3$ [11].

The poles of the nominal- $\pi$ circuit at very low frequencies $\left(10^{-4} \mathrm{~Hz}\right)$ are used to calculate the largest time constant of the exact- $\pi$ circuit. These poles are: $-4.6729 \times 10^{5},-16.446,-5.0496 \pm j 2006.8$. To calculate the largest time constant of the MTL-circuits, modal lumped elements indicated in Table 3.2 are used to form six decoupled circuits for each line energization tests. Using the eigen analysis, the smallest amplitude real part pole for MTL-circuits is calculated as 2.58202 for mode 1-circuit for all of the three test systems. 
Table 3.3: Time-frequency parameters for the DTFS.

\begin{tabular}{l|cccc}
\hline Parameters & RL circuit & Nominal- $\pi$ circuit & Exact- $\pi$ circuit & MTL-circuit \\
\hline$\tau_{m}(\mathrm{~s})$ & 0.021543 & 0.122406 & 0.198035 & 0.387294 \\
$T_{c}(\mathrm{~s})$ & 0.200801 & 0.906842 & 1.436245 & 2.761058 \\
$\Delta f(\mathrm{~Hz})$ & 4.980055 & 1.102728 & 0.696260 & 0.362180 \\
$B_{r}(\mathrm{kHz})$ & 0.464198 & 0.464210 & 0.169231 & 0.169231 \\
$B_{i}(\mathrm{kHz})$ & - & 4.657351 & - & - \\
$B_{\ell}(\mathrm{kHz})$ & - & - & 10 & 10 \\
& & & $0.22(\mathrm{step})$ & $0.22(\mathrm{step})$ \\
$B_{s}(\mathrm{kHz})$ & 0.22 & 0.22 & $0.6(\operatorname{cosine})$ & $0.6($ cosine $)$ \\
$f_{c}(\mathrm{kHz})$ & 0.928396 & 9.314702 & 20 & 20 \\
$N_{s}$ & 187 & 8447 & 28725 & 55222 \\
$\Delta t(\mu s)$ & 1073.80 & 107.36 & 50 & 50 \\
\hline
\end{tabular}

Table 3.3 shows the step by step implementation of the guidelines of Section 3.3 to calculate the $T_{c}$ and $f_{c}$ windows for the DTFS method.

For all methods, the simulation time of interest is considered as $50 \mathrm{~ms}$ (unless otherwise stated). $\tau_{m}$ is calculated from the knowledge of the system poles (poles of the RL and nominal- $\pi$ circuits are shown in Table 3.1). The time window determined for the DTFS is $t_{s i m}+7 \tau_{m}$. The corresponding frequency resolution is $\Delta f=1 / T_{c}$. For the frequency window, $B_{r}$ and $B_{i}$ are calculated from the knowledge of the system poles, $B_{\ell}$ is determined by the length of the transmission line, and $B_{s}$ depends on the shape of the waveform. For the nominal- $\pi$ circuit, we calculate $B_{r}$ by taking the second largest amplitude of the real part of the pole. (We skip the largest real part amplitude pole based on the threshold introduced in Section 3.3 to limit the smallest time step to $1 \mu \mathrm{s}$.) The Nyquist frequency $f_{N y}$ is chosen as the largest of the above bandwidths. This gives $f_{c}=2 f_{N y}$. The total number of samples in the time domain and frequency domain is $N_{s}=T_{c} f_{c}$. The corresponding time step size is $\Delta t=1 / f_{c}$.

For DTFS and NLT with regular sampling the system is solved from 0 to $f_{N y}$ ( $k=0$ to $N_{s} / 2$ ). The negative frequencies half of the spectrum is built by taking the 
complex conjugate of the positive frequencies half. As discussed, the simulation results are valid from $t=0$ to $t=t_{\text {sim }}$ for the DTFS and, for comparisons purposes, only this interval will also be considered for the NLT. The results for a number of case studies are presented next.

\subsubsection{RL Circuit Simulation}

Figure 3.9 shows the short circuit current $i_{s c}$ calculated with all methods for the step voltage source for a time window $T_{c}=50 \mathrm{~ms}$.

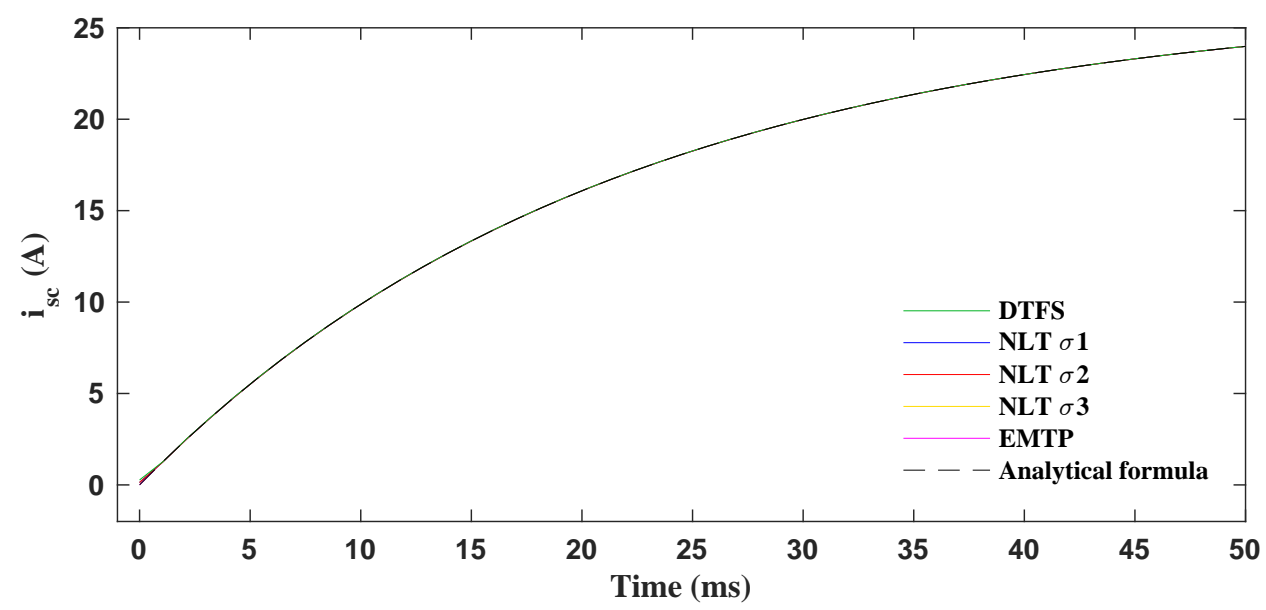

Figure 3.9: $i_{s c}$ for the RL circuit energized with a step voltage.

As observed in Fig. 3.9, the DTFS solution matches very well the NLT, EMTP, and analytical formula. For the simulation time of interest $t_{\text {sim }}=50 \mathrm{~ms}$, the solution given by the NLT did not present any numerical oscillations at the tail of the signal, as a result, the NLT does not require to use windowing function for this simulation.

Maximum absolute error for the DTFS is $0.26 \mathrm{~A}$ and for the NLT (for the three choices of damping factor presented here) is $0.13 \mathrm{~A}$. This error occurs in the first sample for both solutions which is due to the use of forward Euler integration method in the conventional IDTFS. Ignoring the first few samples of the simulation, the average absolute error for the DTFS is $0.006 \mathrm{~A}$ and for the NLT is $0.001 \mathrm{~A}$. This fact signifies that the NLT solution can be very accurate when the optimal choice of damping factor is considered, but only as long as the correct time window width is 
also taken into consideration.

This simulation was run for $t_{\text {sim }}=0.5 \mathrm{~s}$. Figure 3.10 shows the close up of the comparison of DTFS with NLT and EMTP at the tail of the signal. It can be observed that the NLT solution contains oscillations for $t_{\text {sim }}=0.5 \mathrm{~s}$, while for the same simulation time the solution given by the DTFS follows the analytical formula and the EMTP solution very well. Therefore, NLT (unlike the DTFS) requires to use the windowing function to reduce the errors.

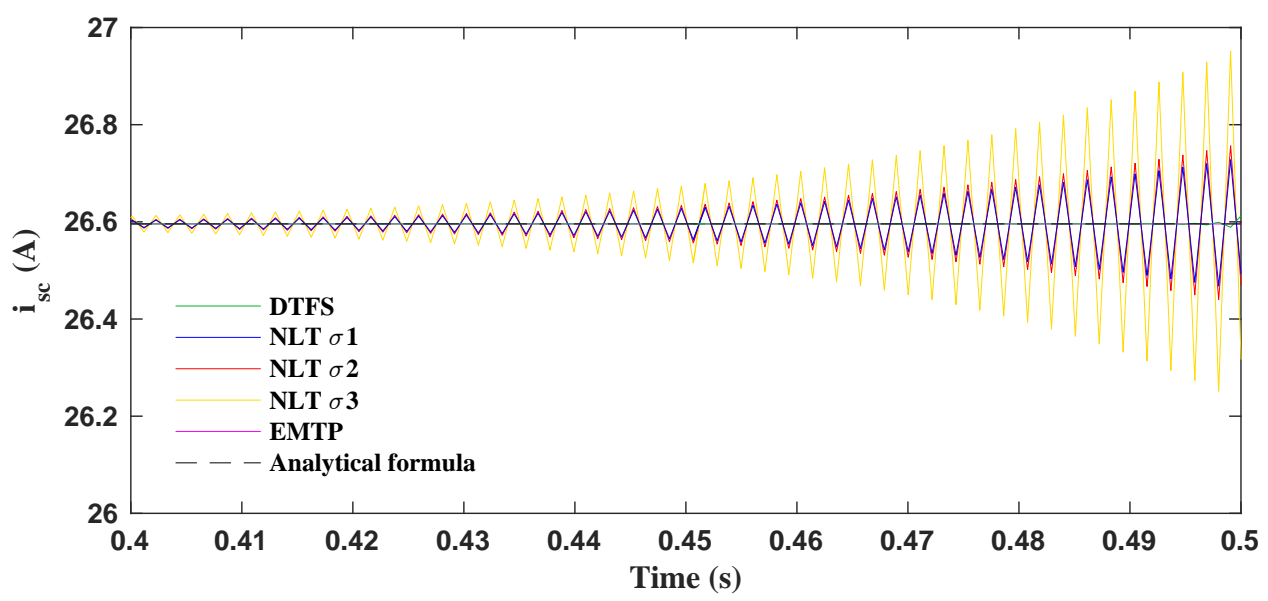

Figure 3.10: Close up of $i_{s c}$ for the RL circuit energized with the step voltage for $t_{\text {sim }}=0.5 \mathrm{~s}$.

Figure 3.11 shows the effect of different windowing functions to reduce the errors of NLT solution in Fig. 3.10. The plot shows the result for NLT with $\sigma_{3}$ (similar results were observed when $\sigma_{1}$ and $\sigma_{2}$ were selected).

It can be observed that Lanczos' window caused minor attenuation to the oscillations since they still show up after the filtering. The filtering effect of Hanning window is stronger than the Lanczos' window, however, it leaves a DC offset after this process which impairs the accuracy of the NLT solution. Tukey window has a better filtering impact with minor superimposed oscillations around the reference analytical formula. However, the DTFS provides a more accurate solution than the NLT without the need to use any windowing functions for this simulation.

Figure 3.12, shows the results for the branch voltage $v_{L}$ in the RL circuit for a step voltage source for $t$ from zero to $t_{\text {sim }}=50 \mathrm{~ms}$ with the same set up considered 


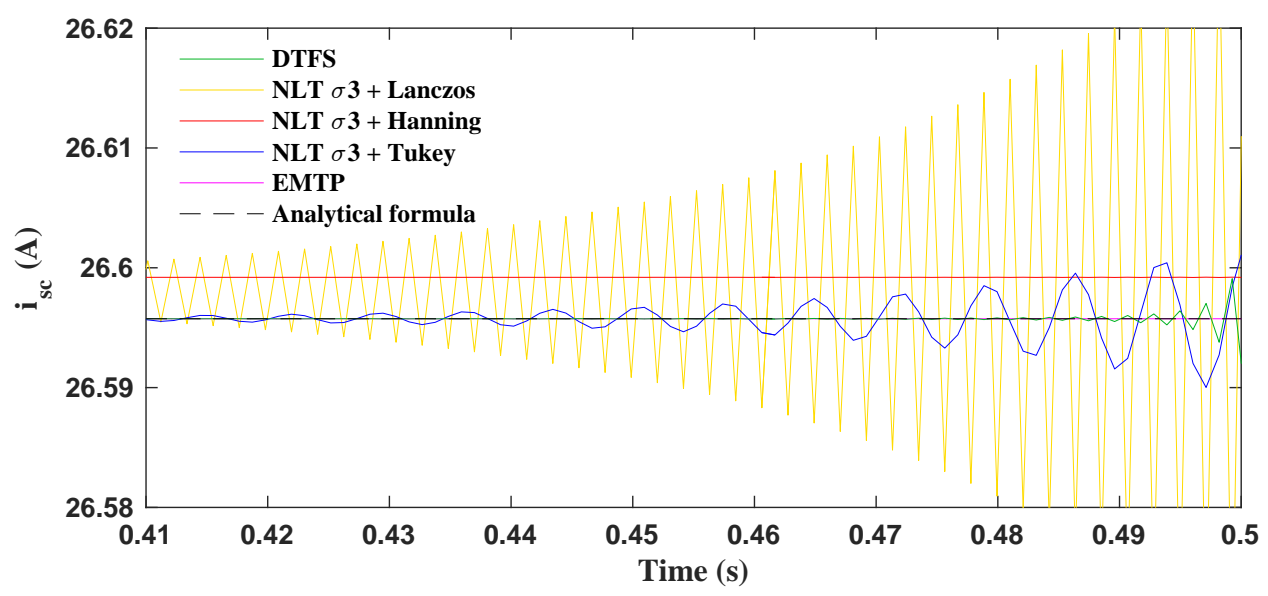

Figure 3.11: Effect of different windowing functions to reduce the oscillations of the NLT solution in Fig. 3.10.

in Fig. 3.11.

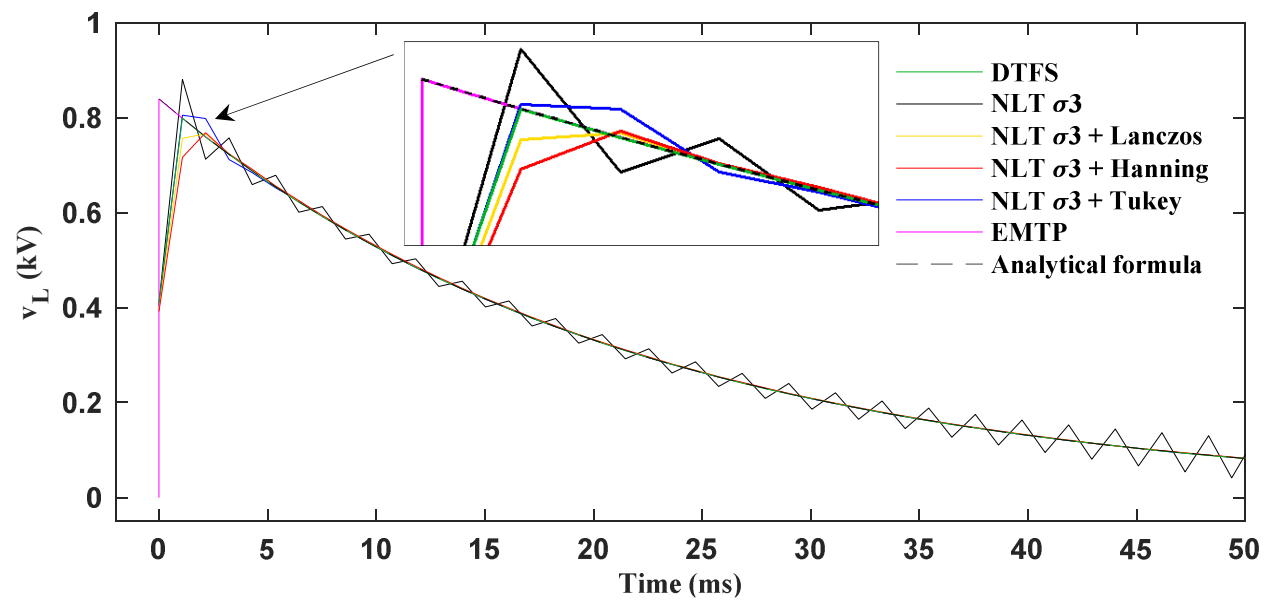

Figure 3.12: $v_{L}$ for the RL circuit energized with the step voltage.

It can be observed that when no filter is applied to the NLT, the solution presents some superimposed Gibbs oscillations, more noticeably, in the beginning and tail portions of the simulation (magnified), while the results for the DTFS, EMTP, and analytical formula are perfectly matched with each other and do not present these oscillations. The cause of error in the NLT is the chopping of frequencies that 
results in Gibbs phenomena. These results illustrate the need for a windowing function in the NLT, while, the proposed DTFS formulation does not require this filtering. The maximum absolute errors for the NLT solution with the application of different windowing functions are: $38 \mathrm{~V}$ for Tukey, $42 \mathrm{~V}$ for Lanczos, and 83 $\mathrm{V}$ for Hanning, while the maximum absolute error for the DTFS is $0.7 \mathrm{~V}$ which is significantly smaller than those for the NLT. Applying Hanning's window to the NLT in this case resulted in an absolute error larger than when no windowing function was used $(81 \mathrm{~V})$. This shows the sensitivity of NLT to the windowing function used.

Ignoring the first few samples, the average absolute error for the NLT is $0.3 \mathrm{~V}$ for Tukey, 0.66 V for Lanczos, and $0.98 \mathrm{~V}$ for Hanning, while this value for DTFS is $0.2 \mathrm{~V}$. The error analysis performed for the simulation shows that for the NLT to gain higher accuracy, we need to search for other choice of damping factors and windowing functions than the conventional ones used in this study.

The scenario of Fig. 3.12 was also used to illustrate the effect of odd sampling in the transient response of NLT. Figure 3.13 compares NLT with odd sampling with DTFS, EMTP, and analytical formula for $t_{\text {sim }}=0.5 \mathrm{~s}$.

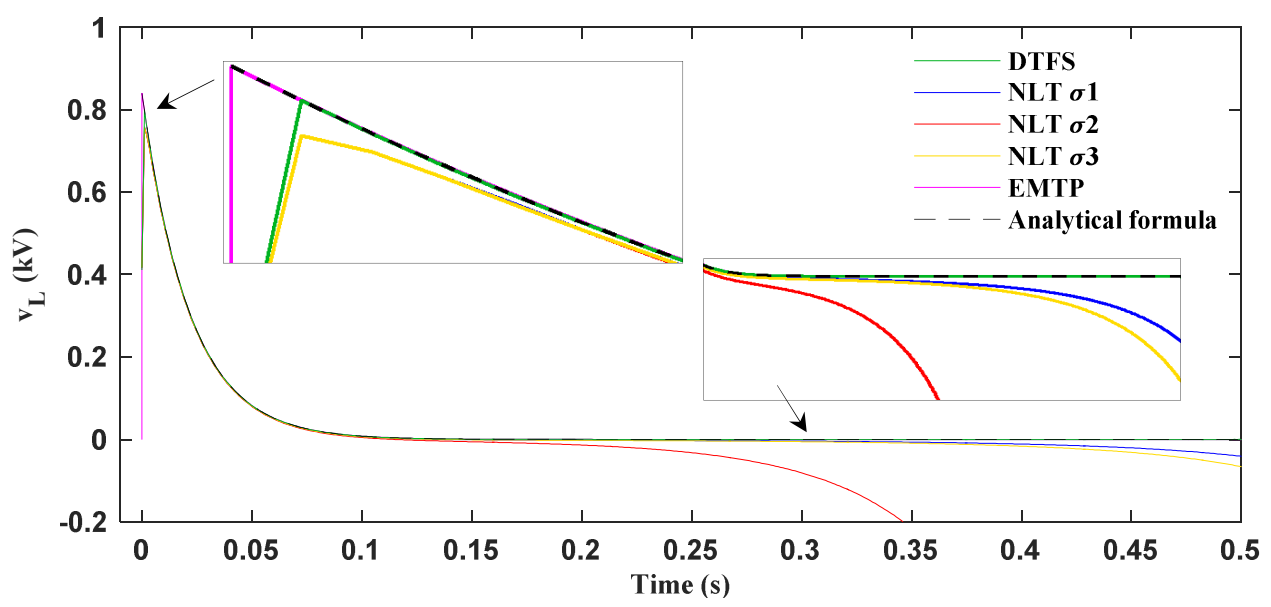

Figure 3.13: Test of NLT with odd sampling in the simulation of $v_{L}$ for the RL circuit

As opposed to DTFS and NLT with regular sampling which solved the system up to $N_{s} / 2$, using the odd sampling for the NLT requires to solve the system for 
the total number of samples $N_{s}$. This happens, as discussed in Section 3.1, due to losing the complex conjugate property in the frequency domain when odd sampling is considered. Therefore, the number of operations is doubled when NLT uses odd sampling.

As observed in Fig. 3.13, the NLT solutions deviate from the EMTP and analytical formula at some point of the simulation while the DTFS perfectly coincides with the reference solutions until $t_{\text {sim }}$ is reached. Similar to the Gibbs oscillations, these deviations for the NLT depend on the value of the damping factor. In this simulation, $\sigma_{1}$ by causing less error offers a better setting for the NLT. Figure 3.13 also shows that NLT underestimates the transient peak by $42 \mathrm{~V}$ for all the damping factors.

Figure 3.14 illustrates the impact of different windowing functions to eliminate the deviation of NLT method in Fig. 3.13.

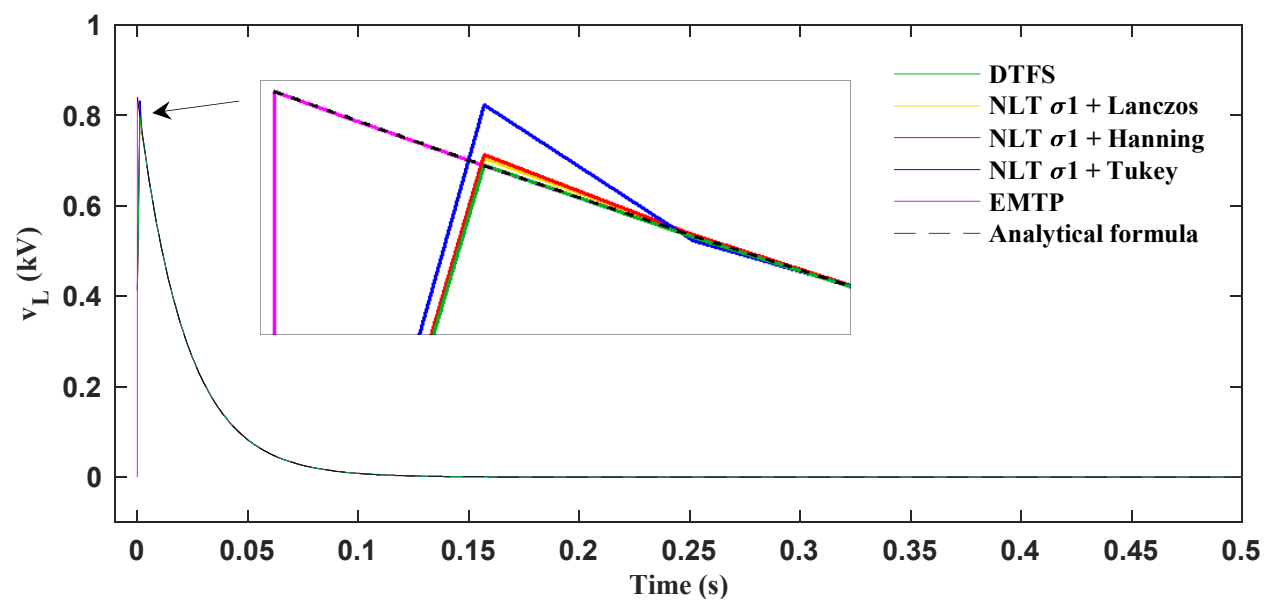

Figure 3.14: The effect of filtering on the accuracy of NLT with odd sampling. Simulation of $v_{L}$ for the RL circuit.

This effect shows up as an overestimation, unlike the results of Fig. 3.13, of the transient peak by $34 \mathrm{~V}$ for Tukey, $5.6 \mathrm{~V}$ for Hanning, and $4.2 \mathrm{~V}$ for Lanczos, while the maximum error of DTFS is $0.7 \mathrm{~V}$. 


\subsubsection{Nominal- $\pi$ Circuit Simulation}

Figure 3.15 shows the simulation results for $i_{s c}$ for the nominal- $\pi$ circuit energization with a step voltage source and $t_{\text {sim }}=50 \mathrm{~ms}$. The results show that the solutions given by all methods are basically identical to each other. The maximum absolute errors are: $4 \mathrm{~mA}$ for DTFS, $1 \mathrm{~mA}$ for NLT with $\sigma_{1}$, and $0.1 \mathrm{~mA}$ for NLT with $\sigma_{2}$ or $\sigma_{3}$, which are all negligible. The NLT did not require to use windowing function for this simulation.

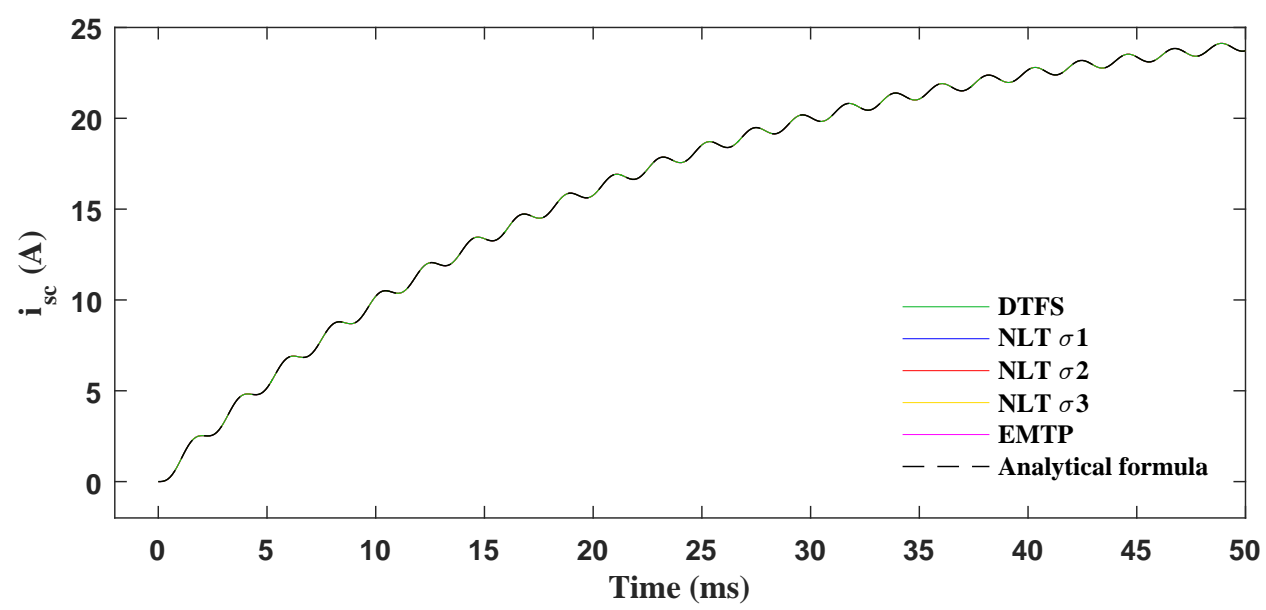

Figure 3.15: $i_{s c}$ for the nominal- $\pi$ circuit energized with the step voltage.

The scenario of Fig. 3.15 was also used to test the DTFS algorithm for the step function input with discontinuities (dashed line in Fig. 3.2b). The solution given by the DTFS without the proposed averaging method is denoted as "DTFS0". Fig. 3.16 is the magnification of Fig. 3.15 for the simulation span between 40 to $45 \mathrm{~ms}$ in which DTFS0 is compared to the rest of solutions. As observed in Fig. 3.16, DTFS0 drifts slightly from the rest of the solutions.

\subsubsection{Exact- $\pi$ Circuit Simulation}

Figures 3.17 and 3.18 show $i_{s c}$ for the exact- $\pi$ circuit calculated with the DTFS, NLT, FDLINE, and ULM for the simulation time of $50 \mathrm{~ms}$. Figure 3.17 shows $i_{s c}$ for a step voltage source, whereas Fig. 3.18 shows the result for a cosine source energization. The results of Figs. 3.17 and 3.18 confirm that the DTFS and NLT 


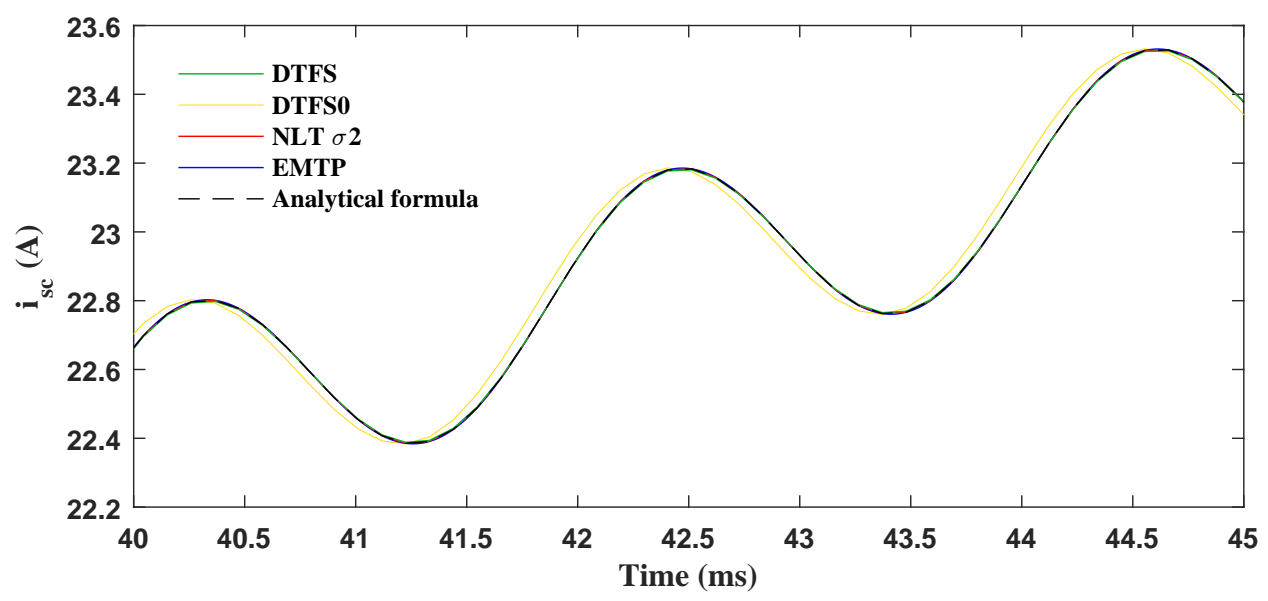

Figure 3.16: Details of Fig. 3.15 illustrating the effect of the first-last point correction.

coincide well with the FDLINE and ULM time domain models in the EMTP.

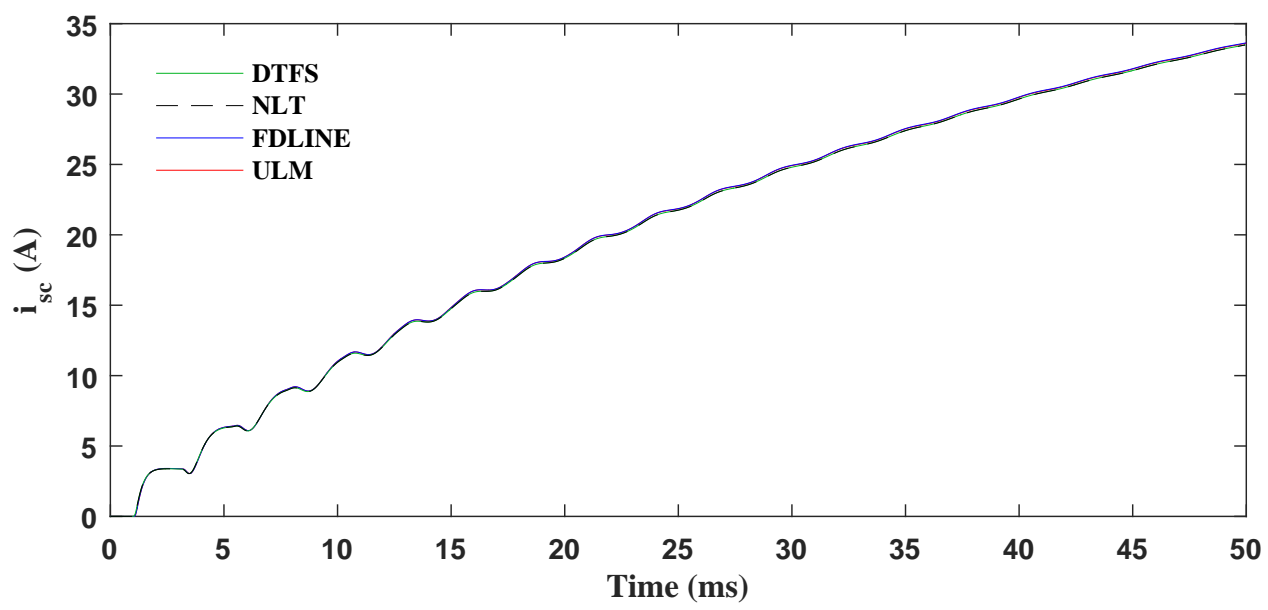

Figure 3.17: $i_{s c}$ for the exact- $\pi$ circuit energized with the step voltage.

An incorrect selection of the time window can lead to aliasing problems in the NLT and to accuracy problems for the proposed DTFS method. Even though the issue of aliasing does not apply to the DTFS because it is a one-to-one mapping between frequency and time, if the system response does not have time to die out within the time window, the DTFS will not include the response to the last 


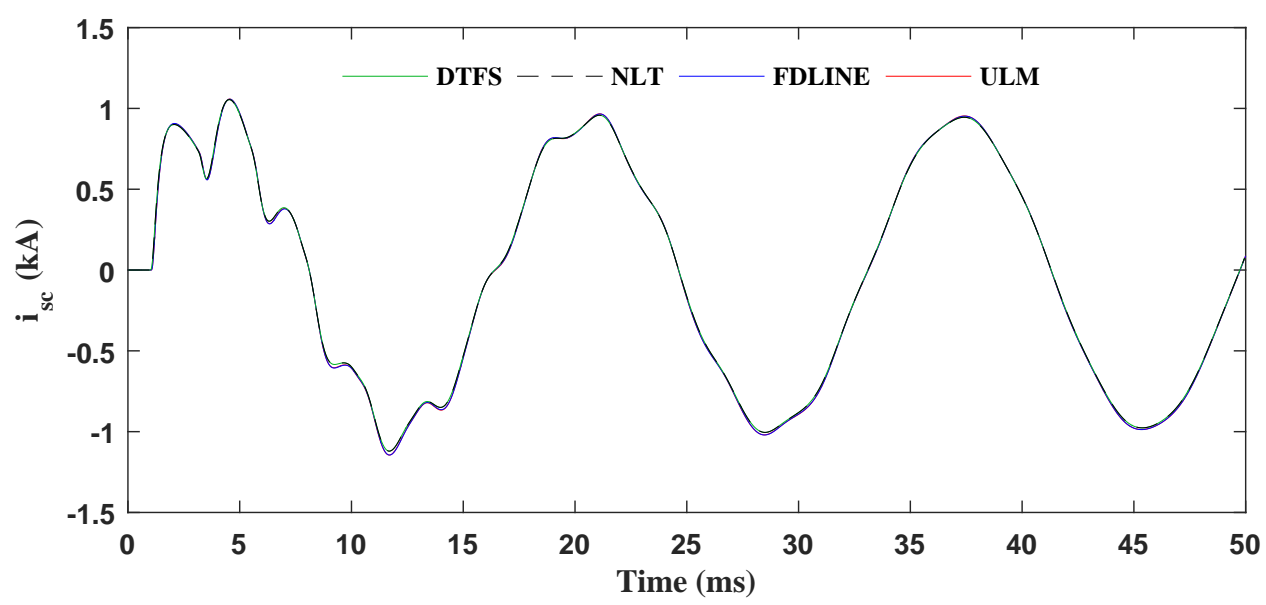

Figure 3.18: $i_{s c}$ for the exact- $\pi$ circuit energized with the cosine voltage.

samples of the source. The example of the exact- $\pi$ circuit energization by a step voltage source with an incorrectly chosen time window can be used to illustrate this problem. Suppose the RL circuit is used to calculate the time constant for the exact$\pi$ circuit at very low frequencies instead of the proposed nominal- $\pi$ circuit. The RL circuit gives a time constant $\tau_{R L}=0.06 \mathrm{~s}$, which is smaller than the time constant obtained with the nominal- $\pi$ circuit $\left(\tau_{N \pi}=0.198 \mathrm{~s}\right)$. Based on the proposed criteria for the calculation of $T_{c}$ in Section 3.3.1, choosing smaller time constant results in reducing the width of the time window.

Figure 3.19 shows a magnification of Fig. 3.17 in which the solution given by the DTFS- $\tau_{R L}$ (DTFS with incorrect $T_{c}$ ) is compared to the DTFS- $\tau_{N \pi}$ (DTFS with correct $T_{c}$ ), NLT, FDLINE and ULM. Choosing an incorrect time window results in an inaccurate results for the DTFS- $\tau_{R L}$, while the results for DTFS- $\tau_{N \pi}$ is perfectly matched with the rest of solutions.

\subsubsection{MTL-Circuits Simulation}

For the simulation of MTL-circuits, difficulties were experienced to set the time window $T_{c}$, damping factor, and sampling scheme for the NLT method.

As mentioned earlier, for benchmarking purposes, the same $T_{c}$ for the DTFS was considered for the NLT solution. The $T_{c}$ consists of $t_{\text {sim }}=0.05 \mathrm{~s}$ and $t_{\text {set }}=7 \tau=2.71$ 


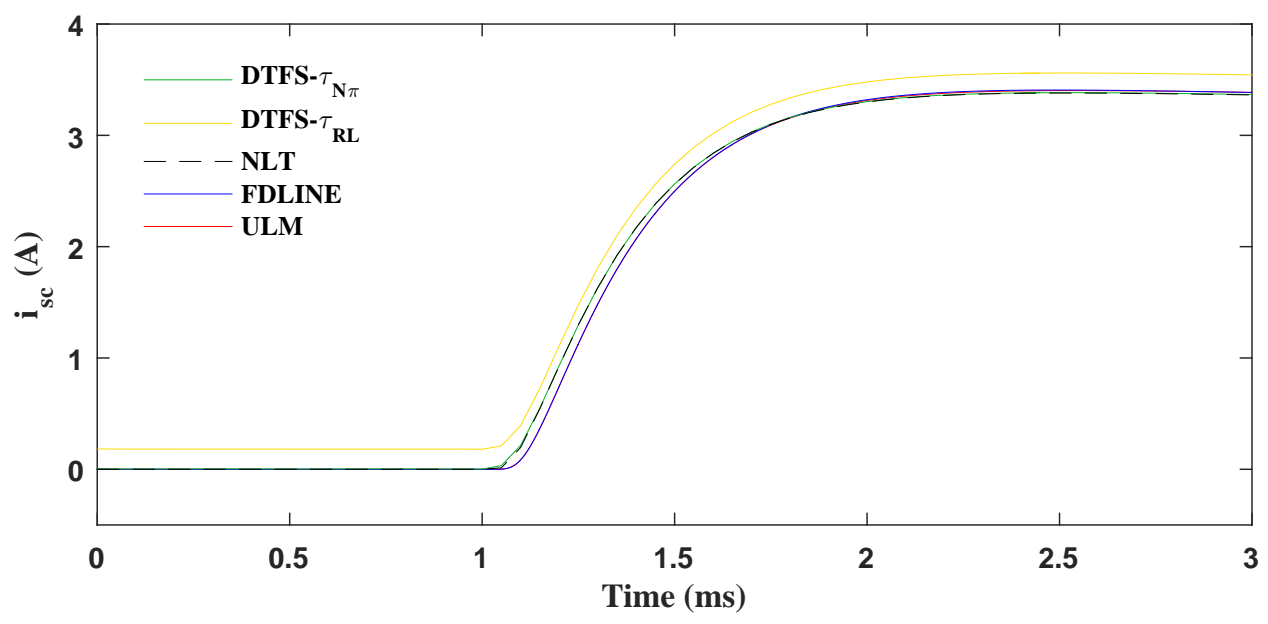

Figure 3.19: Error in the DTFS due to incorrect selection of the time window.

$\mathrm{s}$ which results in $2.76 \mathrm{~s}$ for MTL-circuits. For the same size of $T_{c}$, the NLT solution could match with the DTFS, FDLINE, and ULM solutions only when $\sigma_{1}$ for both regular/odd sampling or $\sigma_{3}$ for odd sampling were selected. Since the operation of regular sampling with the use of complex conjugate property is faster than the odd sampling (89.2 s compared to $171.3 \mathrm{~s}$ for the simulation of Fig. 3.6), $\sigma_{1}$ with regular sampling was considered as the set up for NLT. For other settings to work, the NLT method required to consider a larger time window, as for example: $3.2 \mathrm{~s}$ for $\sigma_{2}$ and $2.79 \mathrm{~s}$ for $\sigma_{3}$ for regular sampling and $3.12 \mathrm{~s}$ for $\sigma_{2}$ for odd sampling. Windowing functions had minor impact to enhance the result of the NLT for its optimum setting with $\sigma_{1}$ or to reduce the size of the typical time windows for other settings.

These observations indicate that obtaining accurate results with the NLT requires experience to choose a correct damping factor. While DTFS is much simpler to implement and does not require this setting.

Figure 3.20 shows the voltage at open conductor 3 of the double circuit vertical line in the unbalanced faults test of Fig. 3.6. In this simulation, the results obtained with the DTFS is compared with NLT, FDLINE, and ULM for $t_{\text {sim }}=50 \mathrm{~ms}$. As can be observed in Fig. 3.20, the solutions given by DTFS and NLT with $\sigma_{1}$ are perfectly superimposed, and these solutions are closely followed by the FDLINE and ULM 


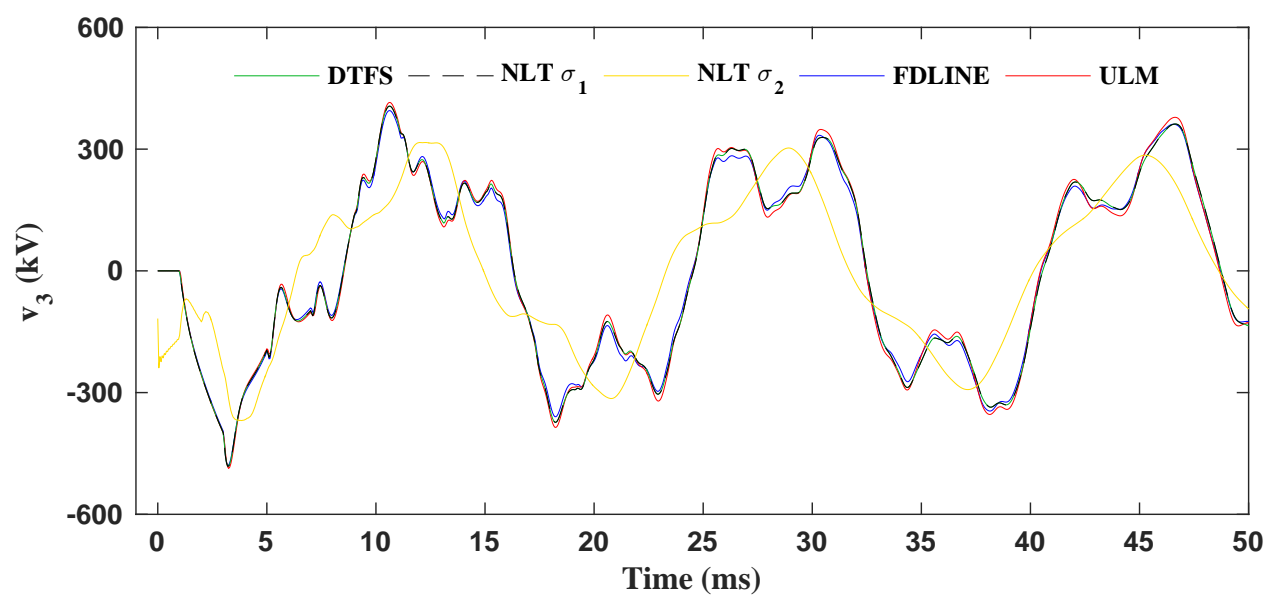

Figure 3.20: $v_{3}$ in the unbalanced faults test of Fig. 3.6.

line models. However, the solution given by NLT with $\sigma_{2}$ does not agree with the rest of the matched results. This conflict shows the sensitivity of the NLT to the size of the time window and the choice of the damping factor. For the rest of the simulations, $\sigma_{1}$ and regular sampling will be considered as the set up for the NLT method.

In Fig. 3.21, short circuit current $i_{6}$ for the double circuit vertical line in the unbalanced faults test of Fig. 3.6 is shown. These results indicate that the solutions given by FDLINE and ULM line models are suitably in agreement with the DTFS and NLT frequency domain solutions.

Figure 3.22 shows the simulations for $v_{6}$ and $i_{2}$ in the induced voltage test of Fig. 3.7 for $t_{\text {sim }}=50 \mathrm{~ms}$. As can be observed, for both $v_{6}$ and $i_{2}$ the DTFS is perfectly matched with the NLT, and both solutions are closely followed by the FDLINE and ULM line models. 


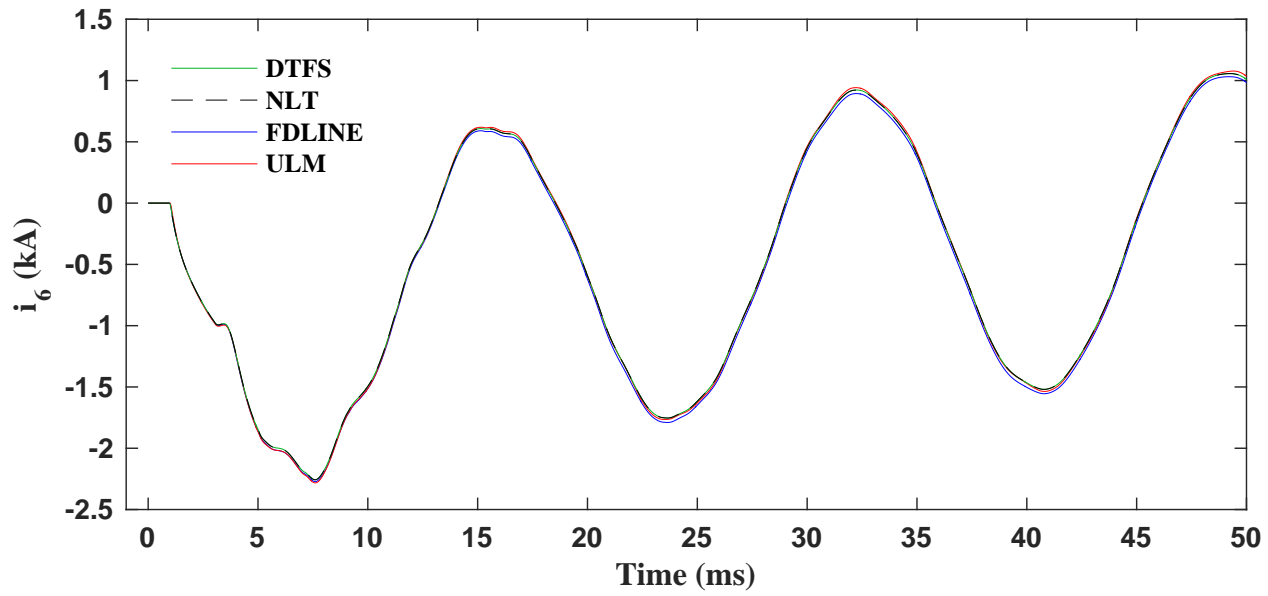

Figure 3.21: $i_{6}$ in the unbalanced faults test of Fig. 3.6.
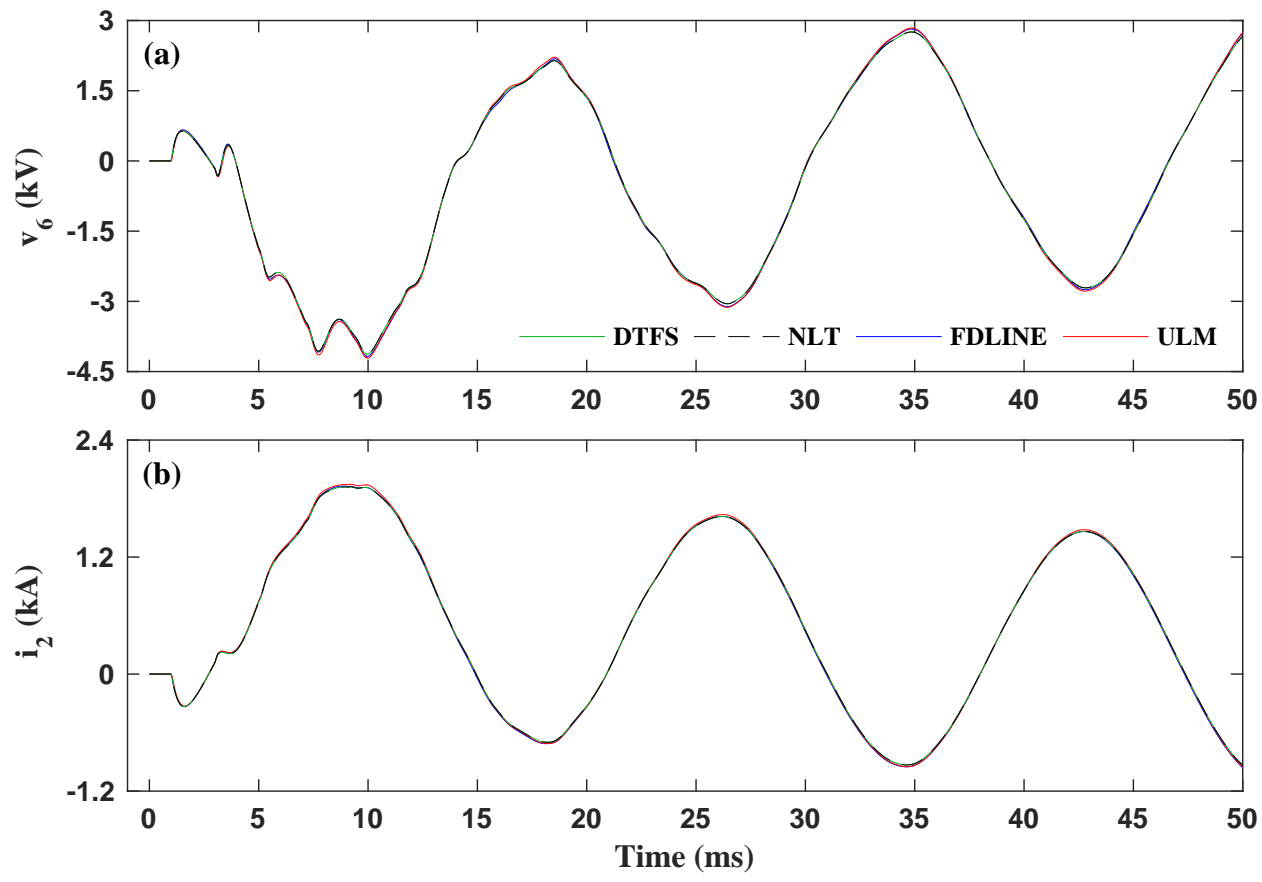

Figure 3.22: $v_{6}$ and $i_{2}$ in the induced voltage test of Fig. 3.7. 
Figure 3.23 shows the solutions obtained with DTFS, NLT, FDLINE, and ULM for the open conductor 1 for the double circuit vertical line in the step response test of Fig. 3.8. In this figure, the results of the DTFS and NLT are suitably superimposed. However, time domain models have minor differences with respect to the frequency domain solutions at the peaks. The maximum error for FDLINE is $17 \mathrm{~V}$ and for ULM is $47 \mathrm{~V}$.

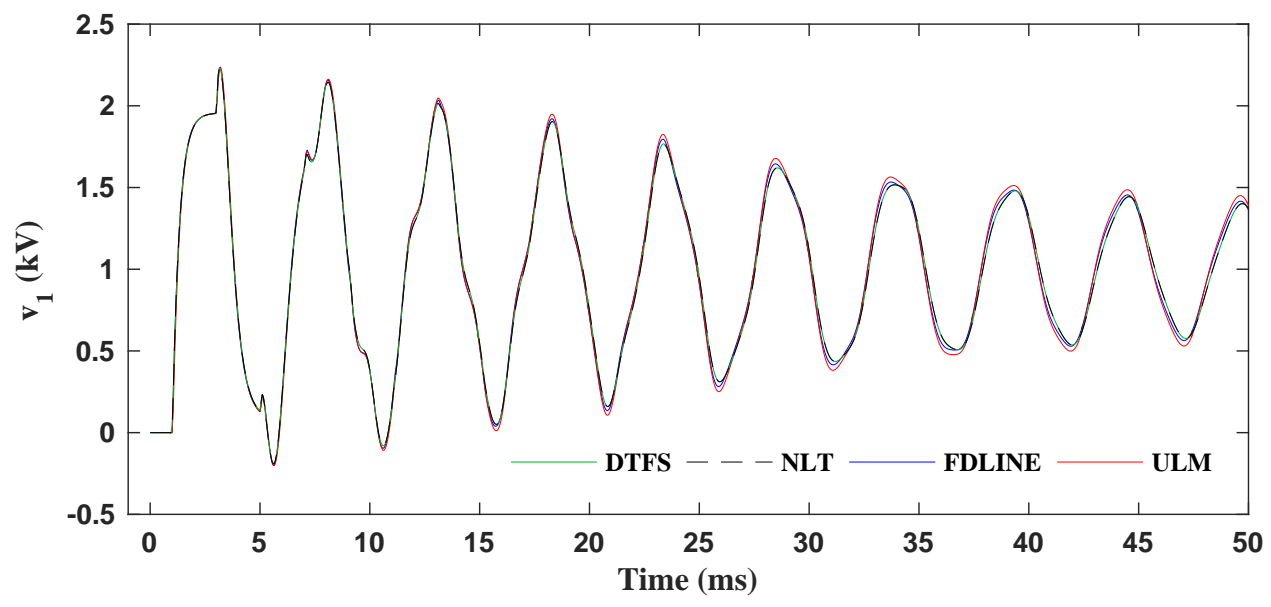

Figure 3.23: $v_{1}$ in the step response test of Fig. 3.8.

Simulation of the step response test of Fig. 3.8 is also used to illustrate the consequence of not correcting the transformation matrices for the modal switching. Figure 3.24a shows the real part of $\mathbf{T}_{I}(\omega)$ for the double circuit vertical line of Fig. 3.5 which contains the modal switching for some elements at $400 \mathrm{~Hz}$ and 2.5 kHz. In Fig. 3.24b, $\mathbf{T}_{I}(\omega)$ was corrected for the modal switchings with the use of Newton-Raphson algorithm [135].

The DTFS was used as an example in this section to address the modal switching problem. However, this is not a typical problem of the DTFS and can also occur for other time and frequency domain solutions. The DTFS solution which is not corrected for the modal switching is denoted as "DTFS-MS".

Figure 3.25 shows the simulations for the voltage at shorted conductor 4 of double circuit vertical line in the step response test of Fig. 3.8. Results are obtained with DTFS-MS (contains modal switching), DTFS (corrected for modal switching), NLT, FDLINE, and ULM. 

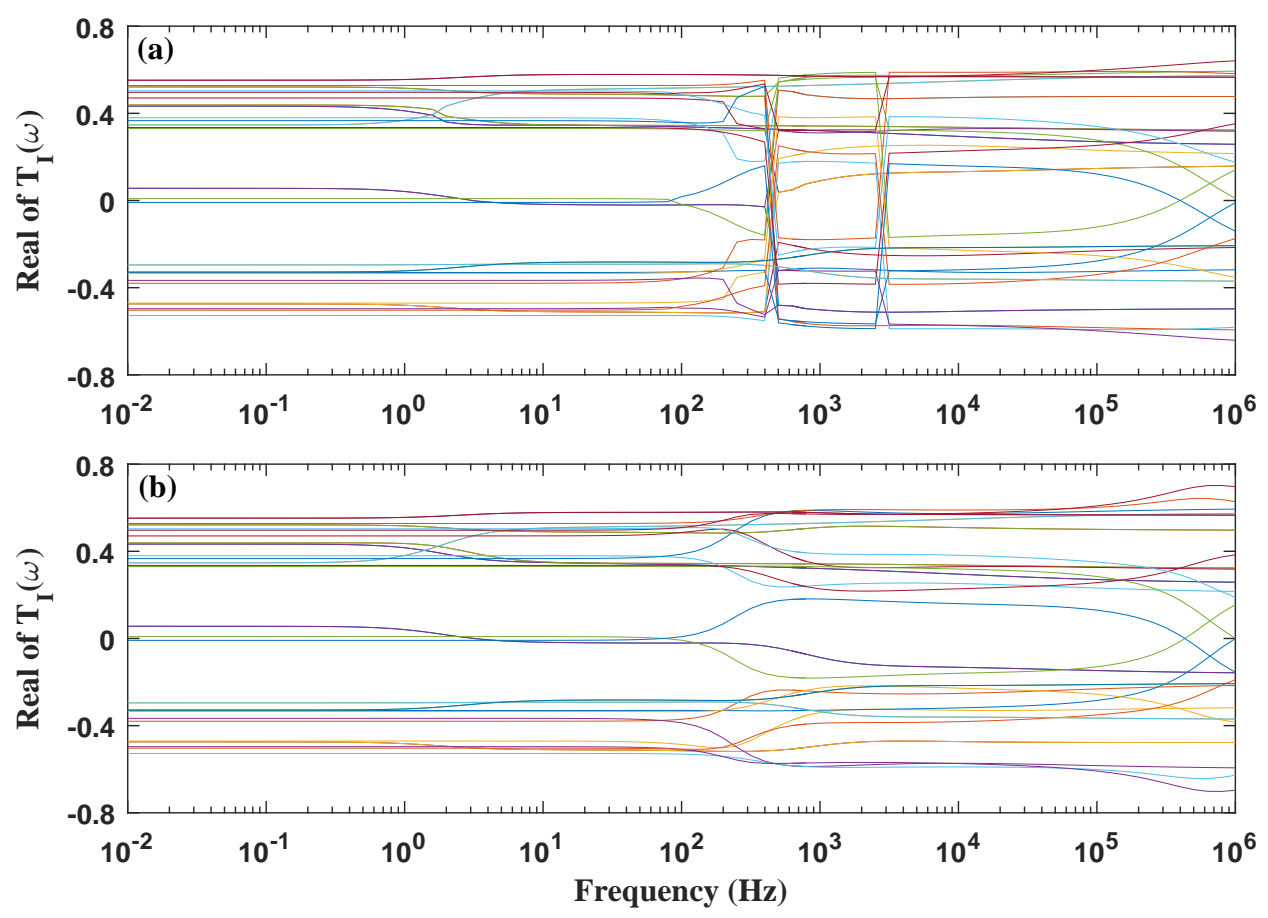

Figure 3.24: The real part of $\mathbf{T}_{I}(\omega)$ for the double circuit vertical line of Fig. 3.5. a) with modal switching. b) corrected for modal switching.

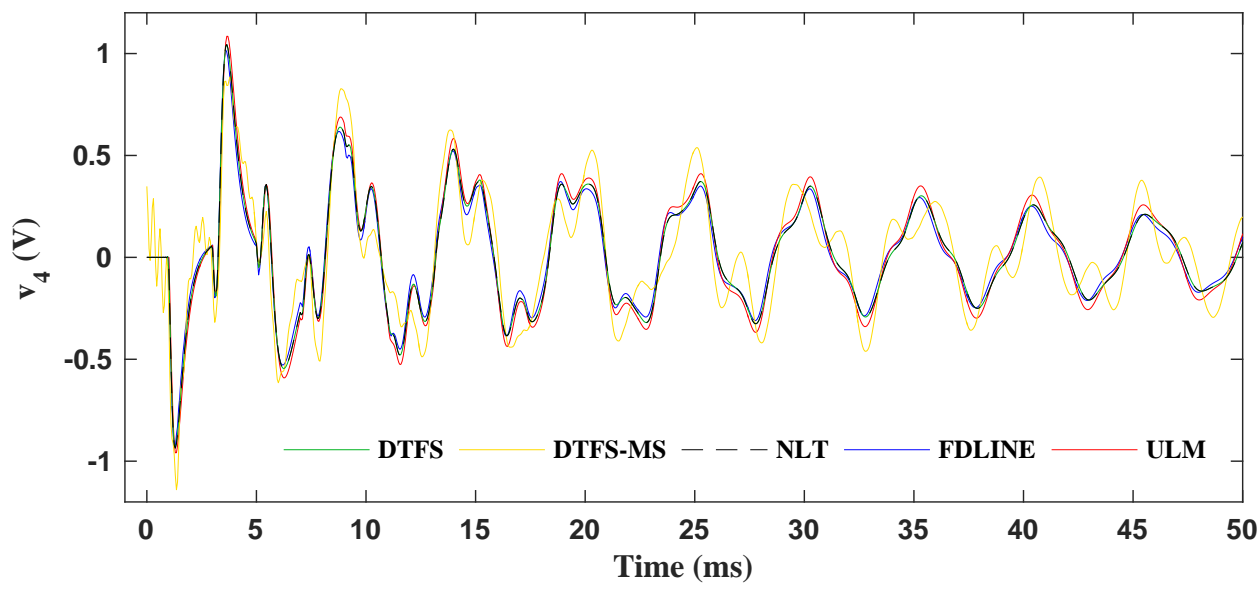

Figure 3.25: $v_{4}$ in the step response test of Fig. 3.8 illustrating the effect of modal switching problem. 
As observed in Fig. 3.25, modal switching appears as high frequency oscillations in the time domain result, more noticeably at the beginning of the signal. Similar to the previous results, the DTFS and NLT solutions are best coincided. FDLINE follows the frequency domain solutions closer than ULM in this simulation. The maximum absolute error for FDLINE is $1.0154 \mathrm{kV}$ and for ULM is 1.0841 $\mathrm{kV}$ compared to the DTFS as a reference.

Overall, by agreeing with the DTFS and NLT solutions, these tests also show that the frequency dependent line models FDLINE and ULM make a very good job in fitting the frequency dependent wave functions in developing their corresponding time domain models.

\subsection{Sensitivity of the DTFS to the Width of the $t_{\text {set }}$}

In this section, a sensitivity analysis is performed to show the accuracy and computational cost of the DTFS method based on the guidelines presented in Section 3.3.1. Figure 3.26a illustrates a relative error of the DTFS method with respect to the reference solution for the width of $t_{\text {set }}$ ranging between 0.1 to $10 \mathrm{~s}$. In Fig. $3.26 \mathrm{~b}$, the computational efficiency of different simulations are illustrated. The test cases were selected from the examples in Section 3.4 which resulted in higher errors:

$-i_{s c}$ in the RL circuit,

$-i_{s c}$ in the nominal- $\pi$ circuit,

$-i_{s c}$ in the exact- $\pi$ circuit energized with the step voltage,

$-v_{3}$ in the double circuit vertical line in the unbalanced faults test.

The reference solutions for the error analysis are the analytical formulas for the RL and nominal- $\pi$ circuits and the solution given by the EMTP line models within the first travelling time (which is zero as shown in Fig. 3.19). The errors are then normalized by the steady state values of the analytical formulas for circuits with lumped elements and EMTP line models for the frequency dependent lines. In Fig. 3.26a, arrows indicate the width $t_{\text {set }}=7 \tau_{m}$ as proposed in Section 3.3.1.

As can be observed in Fig. 3.26a, DTFS has large error when $t_{\text {set }}$ is close to $0.1 \mathrm{~s}$. With the proposed set up for $t_{\text {set }}$, the errors are as follows: $0.7 \%$ for the RL circuit, $0.003 \%$ for the nominal- $\pi$ circuit, $0.006 \%$ for the the exact- $\pi$ circuit, and 
$3.5 \times 10^{-6} \%$ for the double circuit line. Taking $t_{\text {set }}=2 \tau_{m}$ for the double circuit line test results in $1.7 \times 10^{-5} \%$ error.

By comparing Figs. 3.26a to $3.26 \mathrm{~b}$ we can conclude that increasing the width of $t_{\text {set }}$ from the proposed criterion will increase the computational cost at the expense of no significant gain in the accuracy.
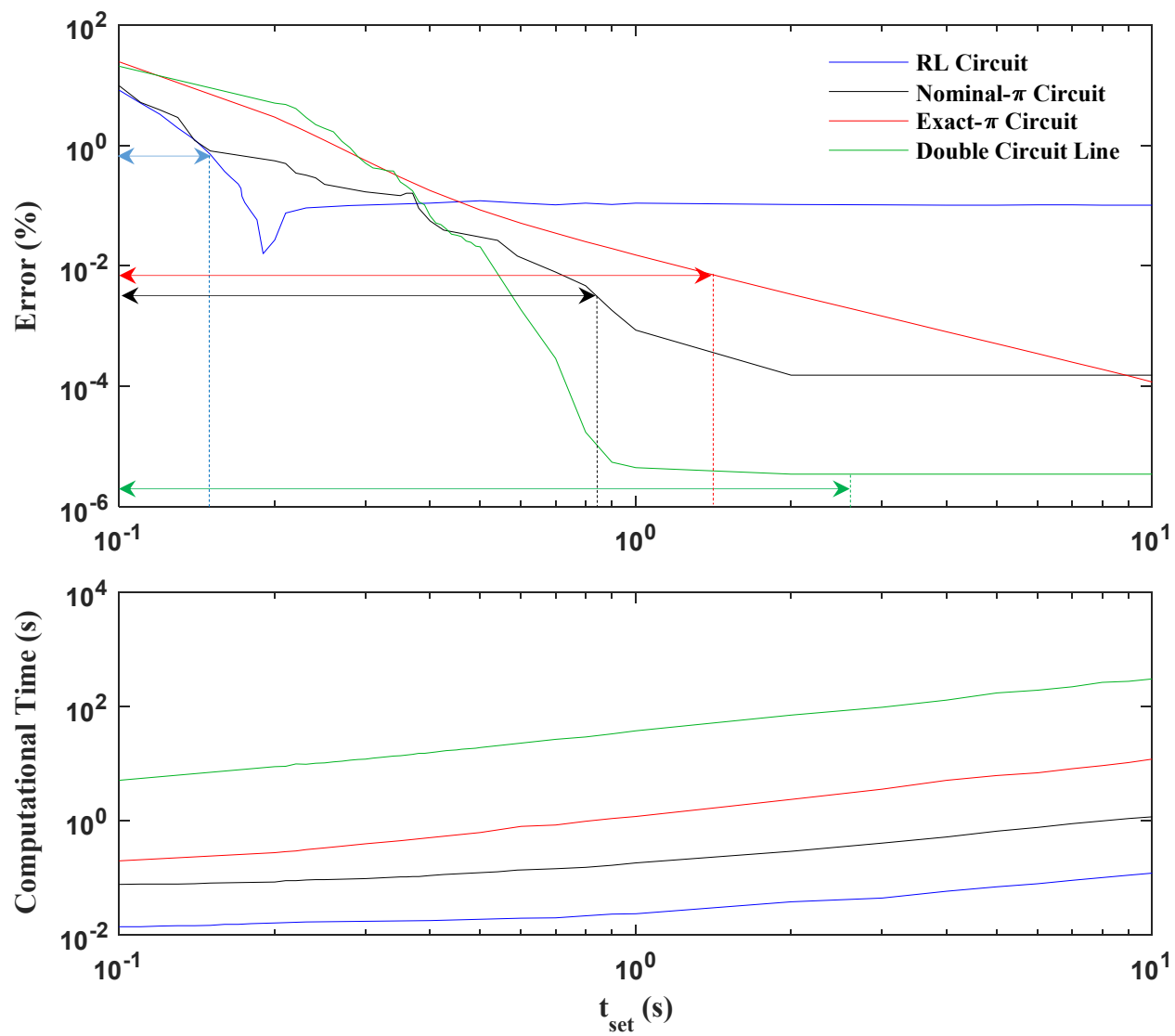

Figure 3.26: Sensitivity of the DTFS method to the width of the $t_{\text {set }}$ for different tests. a) Relative error, b) Computational time.

As discussed in Section 1.1, frequency domain solutions are not as efficient as time domain solutions in terms of the computational cost. Their application is limited to verify the accuracy of the corresponding time domain models. 


\subsection{General Observations}

In this chapter, we proposed a simple methodology to implement a frequency domain solution based on the DTFS for power system transient analysis. The proposed algorithm specifies the time window width based on the slowest time constant of the system and the frequency window width based on the maximum frequency of interest in the transient.

The accuracy of the proposed DTFS was verified by comparing the simulation results with the analytical formulas obtained for circuits with lumped elements. The DTFS solution coincided well with the FDLINE and ULM in the simulation of a double circuit vertical line under asymmetrical line energization and terminal conditions.

Simulation results showed the sensitivity of the NLT solution to the selection of damping factor, windowing function, and time window width. For some simulations in this chapter, similar to the simulation results in Chapter 2, we could not obtain good results with the NLT that compare well with the DTFS and EMTP solutions due to the difficulty to adjust the NLT parameters. While, the DTFS uses a simpler implementation than the NLT which facilitates its usage for non-expert users. Sensitivity analysis performed in Section 3.6 verified the accuracy and computational efficiency of the proposed guidelines for the application of DTFS.

Simulation results for the double circuit vertical line in Section 3.5.4 repeated the conclusion in Chapter 2 that FDLINE, by approximating the transformation matrices at one frequency, is very accurate for the simulation of asymmetrical line

configurations. In the next section, we further investigate the effect of calculating the transformation matrices at different frequencies on the accuracy of the FDLINE.

\subsection{Sensitivity of FDLINE to the Frequency of Approximation for the Transformation Matrices}

The suggested frequency for the calculation of the transformation matrices for FDLINE is $1 \mathrm{kHz}$ [2]. In this section, we perform a sensitivity analysis to find the effect of this frequency on the accuracy of FDLINE.

The double circuit vertical line of Fig. 3.5 in the unbalanced faults test of Fig. 3.6 is chosen as an example for this test. FDLINE is simulated with transformation 
matrices calculated for a frequency range from $10^{-2} \mathrm{~Hz}$ to $10^{6} \mathrm{~Hz}$ with 10 points per decade.

The accuracy of the FDLINE is evaluated by calculating a relative error with respect to the DTFS solution for the transient period and steady state condition. The transient error is calculated as the average of absolute error of FDLINE and DTFS over a period of $0.1 \mathrm{~s}$ where most transient happens. The transient error is normalized by the steady state value of DTFS solution, and it is then scaled by a factor 100. The steady state error is calculated as a relative error of FDLINE and DTFS at $t_{\text {sim }}=0.6 \mathrm{~s}$ where the transient is almost settled. The transient and steady state errors of ULM are also calculated for comparison purposes. Since ULM does not approximate the transformation matrices, these errors are constant over the frequency range of study. Figure 3.27 shows the transient and steady state errors for FDLINE and ULM with respect to the DTFS.

As observed in Fig. 3.27, FDLINE has the highest errors for frequencies smaller than $1 \mathrm{~Hz}$. For the frequency range of $10 \mathrm{~Hz}$ to $10 \mathrm{kHz}$ (including $1 \mathrm{kHz}$ as the traditional setting) FDLINE can be more accurate than ULM for all the simulations, except for $i_{6}$ that ULM is slightly better $(0.88 \%$ error for ULM versus $1.22 \%$ error for FDLINE).

From the results of Fig. 3.27, one can conclude that choosing the frequency at about $60 \mathrm{~Hz}$ can result in minimum transient and steady state errors for FDLINE. For simplicity, we refer to the solution obtained with the traditional setting at 1 $\mathrm{kHz}$ as "FDLINE" and for the new setting at $60 \mathrm{~Hz}$ as "FDLINE*".

In Fig. 3.27, the maximum transient error for the open voltages are $3.72 \%$ for ULM, 3.00\% for FDLINE, $2.69 \%$ for FDLINE*, and for short circuits are $2.13 \%$ for FDLINE, $0.94 \%$ for ULM, $1.22 \%$ for FDLINE*. The maximum steady state errors for both open voltages and short circuit currents are $1.31 \%$ for ULM, $0.81 \%$ for FDLINE, and $0.71 \%$ for FDLINE*. These results indicate that FDLINE can be more accurate when the frequency of approximation for the transformation matrices are calculated at $60 \mathrm{~Hz}$ than the traditional setting at $1 \mathrm{kHz}$. These results were also observed for a variety of other line configurations. 

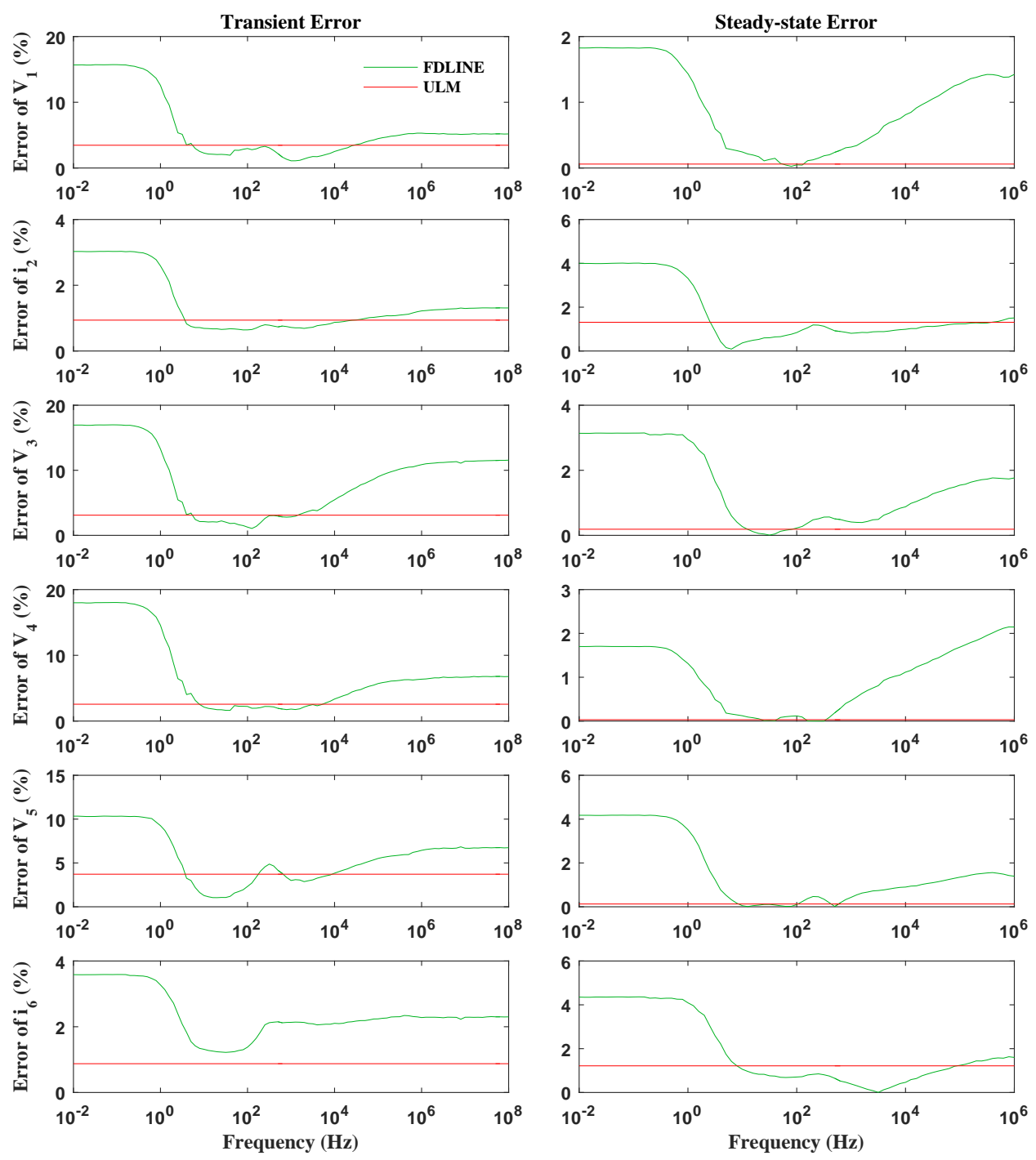

Figure 3.27: Sensitivity of FDLINE to the frequency of approximation

Simulation results of Figs. 3.20-3.23 are magnified in Figs. 3.28-3.30 to illustrate the improvement of FDLINE with the new setting. 
Figure 3.28 shows the close up for $v_{3}$ and $i_{6}$ for the unbalanced faults test of Fig. 3.6. Results clearly show that FDLINE* follows the DTFS solution closer than FDLINE and ULM for both open voltage $v_{3}$ and short circuit $i_{6}$.
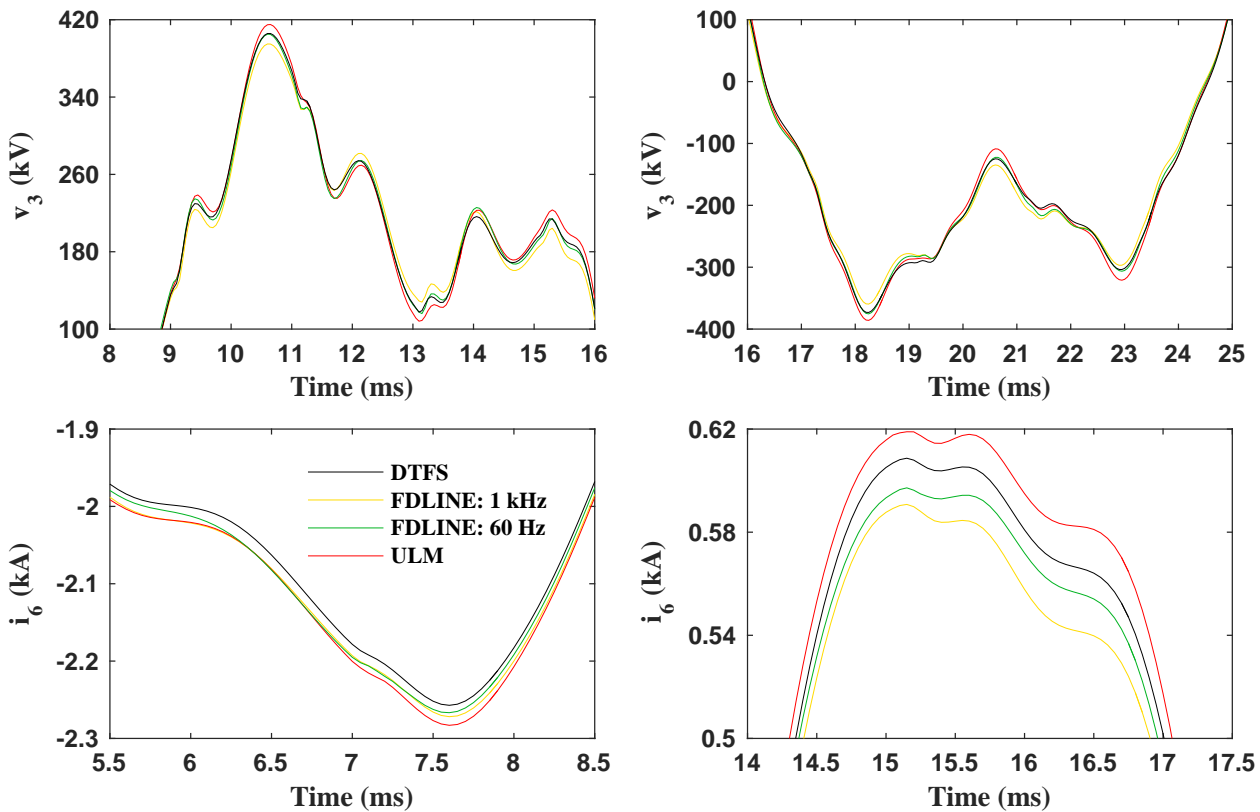

Figure 3.28: Close up of $v_{3}$ and $i_{6}$ in the unbalanced faults test of Fig. 3.6. 
Details of $i_{2}$ and $v_{6}$ for the induced voltage test of Fig. 3.7 are illustrated in Fig. 3.29. As observed in this figure, FDLINE* is slightly closer than FDLINE, and both models are closer than ULM when compared to the DTFS solution.
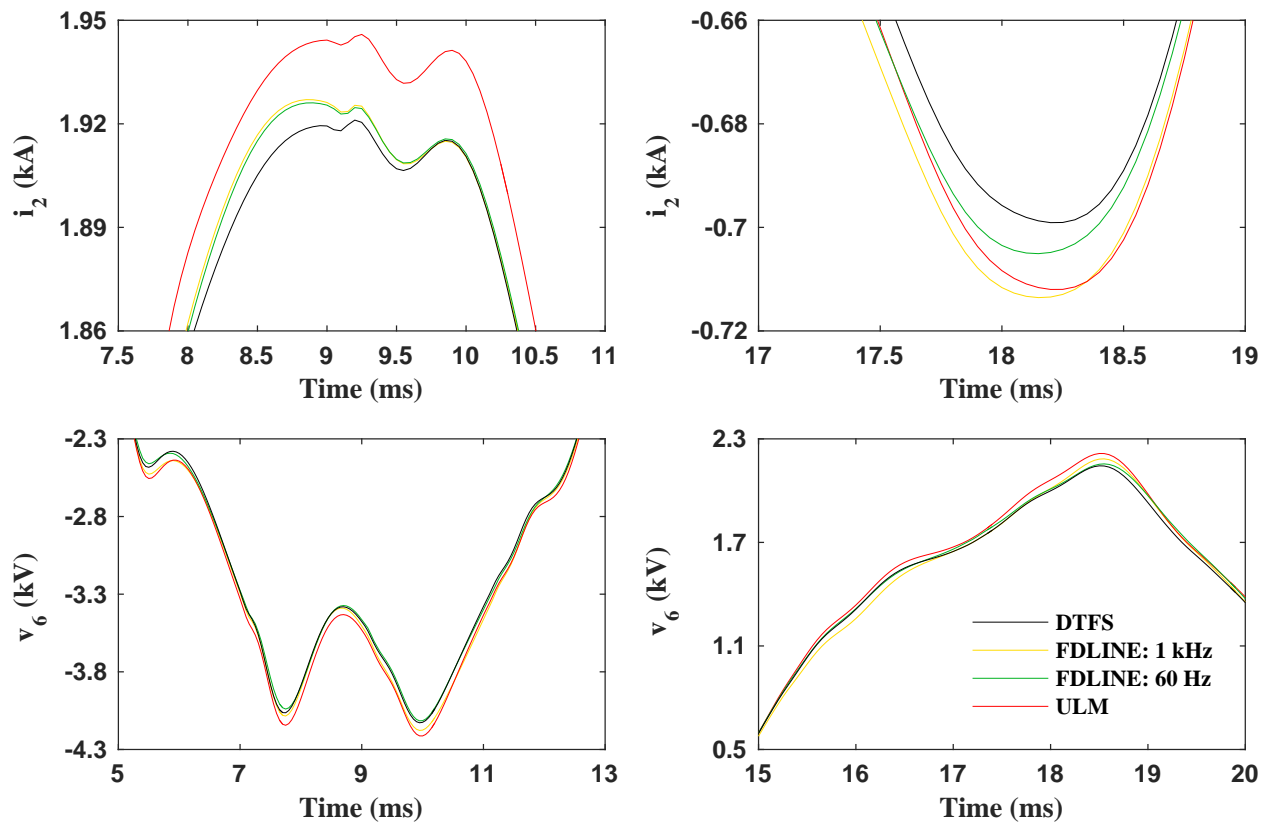

Figure 3.29: Close up of $i_{2}$ and $v_{6}$ in the induced voltage test of Fig. 3.7. 
Figure 3.30 shows the transient solutions for $v_{1}$ in the step response test of Fig. 3.8 for two periods: a) $0<t<20 \mathrm{~ms}$ and b) $0.1<t<0.15 \mathrm{~s}$. As can be observed, for the simulation time of interest up to $20 \mathrm{~ms}$, the solutions obtained with FDLINE and ULM are basically matched with the DTFS solution. In this period, FDLINE is not sensitive to the frequency of approximation. However, as simulation advances, FDLINE* continues to follow the DTFS with minimum errors in the peaks, while ULM and FDLINE solutions include a noticeable phase shift drift with larger errors at the peaks. The deviation of the ULM is larger than FDLINE which is mainly due to sacrificing the minimum-phase shift condition in the fitting functions.
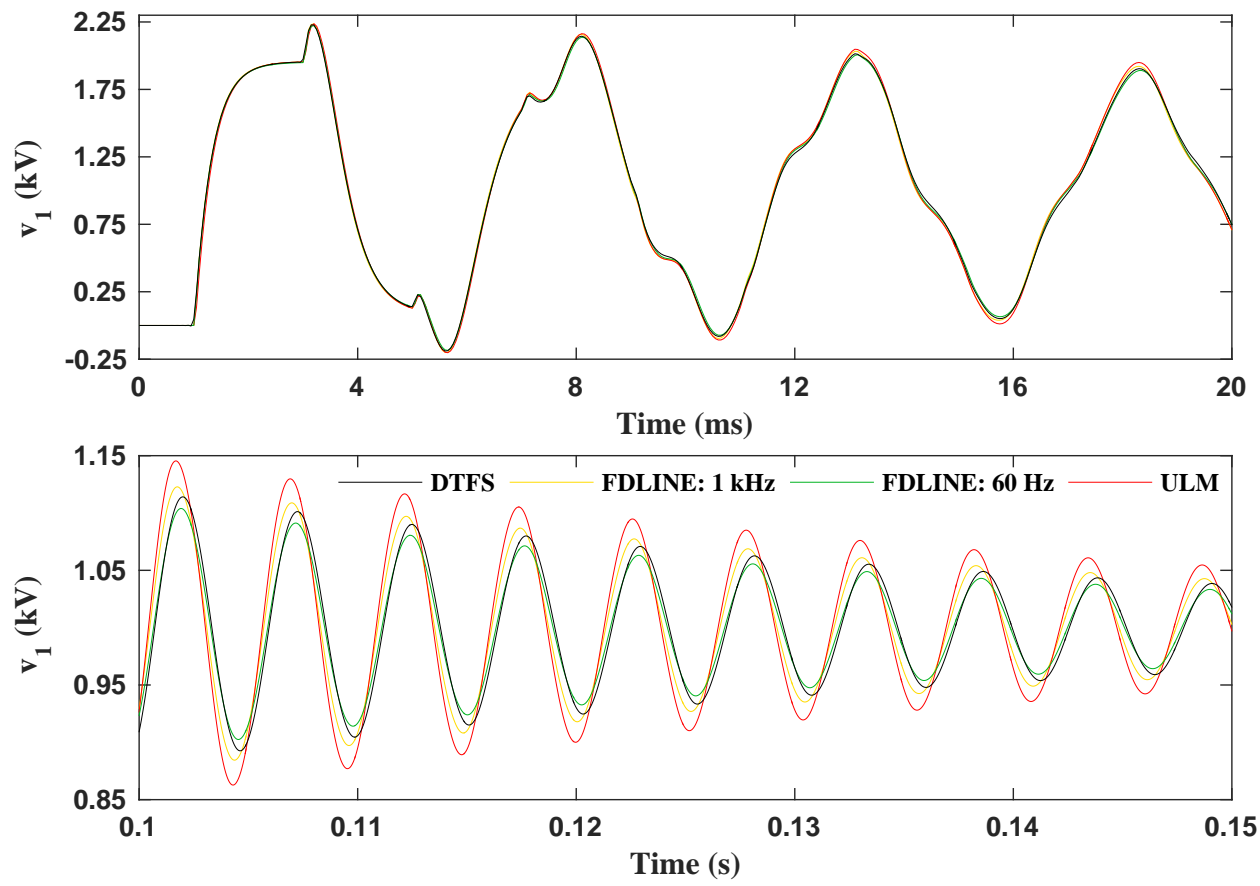

Figure 3.30: $v_{1}$ in the step response test of Fig. 3.8. 


\section{Chapter 4}

\section{Revised Multiconductor}

\section{Transmission Line Equations,}

\section{Proposed Frequency-Dependent Line Model}

This chapter proposes additional physical constraints on the classical MTL equations. The main conceptual premise is that the voltage wave and the current wave should be collocated and travel with the same propagation function along the line. This leads to the analysis of the way the resistances of the ground return path are included in the series impedances. A set of revised equations is proposed RMTL that has the same propagation function for currents and voltages. As opposed to the MTL equations that require complex frequency dependent transformation matrices for their diagonalization, the RMTL equations can be diagonalized using a single real constant transformation matrix. Based on the RMTL equations, a new "frequency dependence simulation" line model "FDLM" is proposed that is much simpler than ULM, while still being fully frequency dependent. The validity of FDLM is verified with comparisons with FDLINE, ULM, and a recently developed DTFS frequency domain solution. 


\subsection{Inconsistencies in the Classical MTL Equations}

As discussed in Chapter 2, the per unit length series impedance and shunt admittance matrices are traditionally calculated in

$$
\begin{aligned}
& \mathbf{Z}=\left(\mathbf{R}_{c}+\mathbf{R}_{e}\right)+j \omega \mathbf{L}_{\text {loop }} \\
& \mathbf{Y}=\mathbf{G}_{\text {ins }}+j \omega \mathbf{C}_{\text {ext }}
\end{aligned}
$$

The wave propagation constants $\gamma_{V}$ and $\gamma_{I}$ are calculated from the series impedance and shunt admittance of $(4.1)$,

$$
\begin{aligned}
& \gamma_{\mathbf{V}}=\sqrt{\mathbf{Z Y}}=\sqrt{\left(\mathbf{R}_{c}+\mathbf{R}_{e}+j \omega \mathbf{L}_{\text {loop }}\right)\left(\mathbf{G}_{\text {ins }}+j \omega \mathbf{C}_{\text {ext }}\right)} \\
& \gamma_{\mathbf{I}}=\sqrt{\mathbf{Y Z}}=\sqrt{\left(\mathbf{G}_{\text {ins }}+j \omega \mathbf{C}_{\text {ext }}\right)\left(\mathbf{R}_{c}+\mathbf{R}_{e}+j \omega \mathbf{L}_{\text {loop }}\right)}
\end{aligned}
$$

In general, the product of two matrices is not commutative, and it is assumed in the classical MTL solution (e.g., [126][93]) that

$$
\mathbf{Z Y} \neq \mathbf{Y Z}
$$

If (4.4) were to be true, then

$$
\gamma_{V} \neq \gamma_{I}
$$

In a conceptual experiment, let us assume that we apply an excitation to a multiconductor uniform semi-infinite transmission line. For this line, there will be no return waves and the voltage and current propagation equations (2.15) and (2.16) will only have forward components,

$$
\mathbf{V}_{f}(x)=\mathbf{V}_{f k} e^{-\gamma_{v} x} \quad, \quad \mathbf{I}_{f}(x)=\mathbf{I}_{f k} e^{-\gamma_{\mathbf{I}} x}
$$

where $\mathbf{V}_{f}(x)$ and $\mathbf{I}_{f}(x)$ are related as in (4.7).

$$
\mathbf{V}_{f}(x)=\mathbf{Z}_{c} \mathbf{I}_{f}(x)
$$

A fundamental assumption of TEM propagation, on which the MTL equations are based, is that in a long uniform transmission line, there are no waves propagat- 
ing perpendicular to the line and the longitudinal propagation equations (4.8) are sufficient to describe the line.

$$
\frac{\partial^{2} \mathbf{V}}{\partial x^{2}}=(\mathbf{Z Y}) \mathbf{V} \quad, \quad \frac{\partial^{2} \mathbf{I}}{\partial x^{2}}=(\mathbf{Y Z}) \mathbf{I}
$$

This assumption (e.g., [93]), requires that skin effect in the capacitances be ignored and that $\mathbf{C}$ remains constant at its electrostatic value $\mathbf{C}_{\text {ext }}$. This is the assumption made in the existing EMTP line models.

The ratio, in a matrix sense, between the voltage wave and the current wave is the characteristic impedance matrix $\mathbf{Z}_{c}$ of (4.9).

$$
\mathbf{Z}_{c}=\mathbf{Y}^{-1} \sqrt{\mathbf{Y Z}}
$$

Since the line is assumed to be uniform and infinite, the $\mathbf{Z}_{c}$ that relates the voltage and current waves at any point $x$ of the line should be the same. However, this is not enforced in the classical MTL equations. Relating in (4.6), $\mathbf{V}_{f}(x)$ and $\mathbf{I}_{f}(x)$, with the assumption that $\gamma_{V} \neq \gamma_{I}$, it follows that

$$
\mathbf{V}_{f}(x)=\left[\mathbf{Z}_{c} e^{-\left(\gamma_{V}-\gamma_{I}\right) x}\right] \mathbf{I}_{f}(x)
$$

In this case then the relationship between the forward voltage wave and the forward current wave becomes a function of the position $x$, which contradicts the physical assumption of a semi-infinite uniform line, where we should be able to apply a given voltage at any point $x$ and get the same current. Propagation according to (4.10) makes the voltage and current waves separate from each other as the wave advances, that is, to lose "collocation". The problem of enforcing, at least the property of symmetry in the product of the characteristic admittance and the propagation function is studied in [108]. In this study, however, we go beyond symmetry, and require that the characteristic impedance does not change anywhere in the semi-infinite line and, therefore, that $\gamma_{V}-\gamma_{I}$ in (4.10) be zero.

\subsection{Collocation of Current and Voltage Waves}

To resolve the physical inconsistency of (4.10), we postulate that for a uniform semi-infinite transmission line in an isotropic medium, (4.2) and (4.3) must be 
equal to each other, regardless of the mathematical argument that the product of two matrices is in general (but not necessarily) non-commutative. If we impose the condition $\gamma_{V}=\gamma_{I}$, we obtain, for the forward wave in (4.10), and with a symmetrical argument for the corresponding backward wave,

$$
\mathbf{V}_{f}(x)=\mathbf{Z}_{c} \mathbf{I}_{f}(x) \quad, \quad \mathbf{V}_{b}(x)=-\mathbf{Z}_{c} \mathbf{I}_{b}(x)
$$

In (4.11) current and voltage are always related by the same $\mathbf{Z}_{c}$, independently of where the waves are in the line. We refer to $(4.11)$ as the wave collocation condition. For this condition to be true, it is required that the matrix product of (4.4) be commutative, that is,

$$
\mathbf{Z Y}=\mathbf{Y Z}
$$

Next, we will look into factors that can be used to enforce the condition (4.12) in the MTL equations.

\subsection{Effect of the Earth Return in the Matrix of Impedances}

As discussed in Chapter 2 and with reference to Fig. 2.2, self and mutual elements of the complex loop inductance $\mathbf{L}_{\text {loop }}^{\prime}$ are calculated in

$$
L_{\text {loop } 11}^{\prime}=\frac{\mu}{2 \pi} \ln \frac{2\left(h_{1}+\bar{p}\right)}{r_{1 e q}} \quad, \quad L_{\text {loop } 12}^{\prime}=\frac{\mu}{2 \pi} \ln \frac{D_{12^{\prime}}}{d_{12}}
$$

The imaginary part of the complex loop inductances of (4.13) are used to calculate the ground return resistances,

$$
\begin{aligned}
& R_{e 11}=j \omega\left(j \mathfrak{I}\left(L_{\text {loop } 11}^{\prime}\right)\right)=-\omega \mathfrak{I}\left(L_{\text {loop } 11}^{\prime}\right) \\
& R_{e 12}=j \omega\left(j \mathfrak{I}\left(L_{\text {loop } 12}^{\prime}\right)\right)=-\omega \mathfrak{I}\left(L_{\text {loop } 12}^{\prime}\right)
\end{aligned}
$$

If the height of conductor 2 in Fig. 2.2 is different from the height of conductor 1, the values for self and mutual loop inductances would be different in (4.13). As a result

$$
R_{e 11} \neq R_{e 12}
$$


Other methods (e.g., [13], [118]) can be used to calculate the values of the $R_{e}$ 's, however, the values obtained are also different for the selves and the mutuals.

Notice that $R_{e 11}$ and $R_{e 12}$ in (4.14) have to be physically the same, even though the formulas will give slightly different results.

To illustrate this conflict, we consider the test circuit of Fig. 4.1 for the line of Fig. 2.1 which shows two conductors 1 and 2 and ground return (it is assumed that the other conductors are open). Assume $\Delta x=1 \mathrm{~m}$ to determine elements $Z_{11}$ and $Z_{12}$ in $\Omega / \mathrm{m}$ for the series impedance matrix.

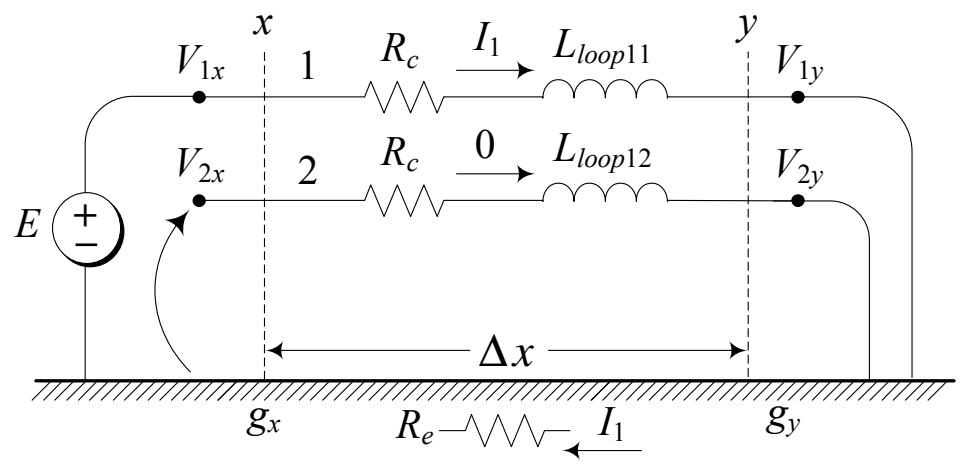

Figure 4.1: Test circuit to determine the self and mutual impedances in the series impedance matrix $\mathbf{Z}$.

Assume that at position $y$ both conductors are shorted to ground and that at position $x$, a source $E$ is connected to conductor 1, while conductor 2 is open, In this test circuit, current $I_{1}$ flows in conductor 1 and returns through ground. With respect to the voltage drop equations (2.1), we will have

$$
V_{1 x}-V_{1 y}=E=Z_{11} I_{1} \quad, \quad V_{2 x}-V_{2 y}=V_{2 x}=Z_{12} I_{1}
$$

and from the circuit solution,

$$
\begin{gathered}
Z_{11}=\left(R_{c}+R_{e}\right)+j \omega L_{\text {loop } 11} \\
Z_{12}=R_{e}+j \omega L_{\text {loop } 12}
\end{gathered}
$$

Since in Fig. 4.1, conductor 2 is not carrying any current, the only resistance contributing to $Z_{12}$ is the resistance of the earth circuit $R_{e}$ encountered by $I_{1}$. No- 
tice also that in this experiment, $R_{e}$ in (4.18) is the same $R_{e}$ in (4.17) because the corresponding voltage drop is due to the same current $I_{1}$ flowing in the same earth path.

We can repeat the tests in Fig. 4.1 for the other elements of the impedance matrix. Assume, for example, that we swap the terminal conditions in conductors 1 and 2, and we inject a current into conductor 2 and measure the voltage in conductor 1 . In this experiment, we will get $Z_{22}$ and $Z_{21}$.

If we now require, as in the first experiment, that the $R_{e}$ for the mutual $Z_{12}$ be the same as for $Z_{22}$, this will make $Z_{12}$ different from the value $Z_{21}$ obtained in the first experiment. This will contradict the condition of symmetry in an isotropic medium, that is, $Z_{12}=Z_{21}$.

Here, to be consistent with the description on $N$ conductors with a common ground return in the MTL equations, we will make all values of $\mathbf{R}_{e}$ equal to the average of all contributions to $\mathbf{Z}$ at each frequency $\bar{R}_{e}$ and still use the slightly non-physical approximations of (4.13) for the calculations.

Figure 4.2 shows elements of the earth resistance matrix $\mathbf{R}_{e}$ for the double circuit vertical line of Fig. 3.5 for a frequency range $10^{-2} \mathrm{~Hz}$ to $10^{6} \mathrm{~Hz}$. In Fig. 4.2, $\mathbf{R}_{e}$ given by (4.14) for the MTL equations are illustrated with green curves, while the black curve is the average of the green curves to be used by the RMTL equations. The maximum difference between the elements of $\mathbf{R}_{e}$ in MTL model are used to calculate an error which is shown with the red curve in Fig. 4.2. This error is normalized by the average value of all contribution of $\mathbf{R}_{e}$ and further expressed in percentage. As can observed in Fig. 4.2, for the normal range of transients in power systems 1 to $10 \mathrm{kHz}$, these differences are within $10-25 \%$.

By factoring out the identical frequency dependent $\bar{R}_{e}$ from the matrix of ground resistance, the remaining matrix can be represented as "all-ones" matrix for an $\mathrm{N}$ conductor system $[\mathbf{1}]_{N \times N}$. With this description, matrix of impedance $\mathbf{Z}$ in the RMTL model can be expressed as

$$
\mathbf{Z}=\mathbf{R}_{c}+\bar{R}_{e}[\mathbf{1}]+j \omega \mathbf{L}_{\text {loop }}
$$

where $\mathbf{R}_{c}$ is a diagonal matrix, $[\mathbf{1}]$ is an $N \times N$ matrix of ones, and $\mathbf{L}_{\text {loop }}$ is a full matrix. 


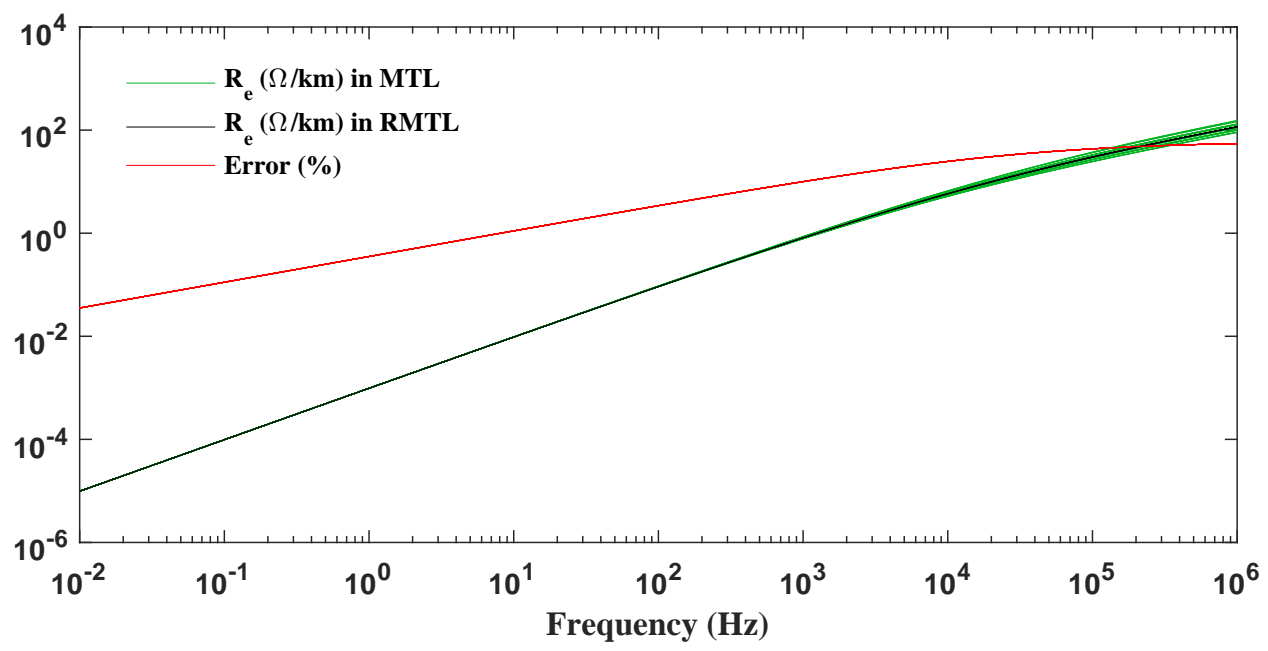

Figure 4.2: Comparison of $\mathbf{R}_{e}$ in MTL and RMTL models for the double circuit vertical line of Fig. 3.5.

\subsection{Validity of the Collocation Condition $\mathrm{ZY}=\mathrm{YZ}$}

The plots in Fig. 4.3 show the validity of the wave collocation condition for the double circuit line of Fig. 3.5 that

$$
\mathbf{Z Y}(\mathbf{Y Z})^{-1}=\mathbf{I}
$$

where, I refers to the identity matrix in this chapter. Ideally, the diagonals should be one and the off-diagonals should be zero.

The curves in Fig. 4.3 are obtained using the conventional $\mathbf{Z}$ and $\mathbf{Y}$ parameters for the MTL equations and also with the ground resistances $\mathbf{R}_{e}$ taken as equal for all entries in the $\mathbf{Z}$ matrix for the RMTL equations.

Both sets of curves are very close, except that enforcing the collocation condition improves (reduces) the imaginary part of all elements of the matrix (Fig. 4.3-bottom). As it can be seen in the plots, the maximum errors in the diagonals are about $2 \%$ and in the off-diagonals about $5 \%$.

These results suggest that it is the nature of the system to satisfy the collocation property, and that there might be some physical inconsistencies in the equations or in the determination of the parameters of these equations that prevent the collocation condition to be exact. The new RMTL equations proposed in this chapter 

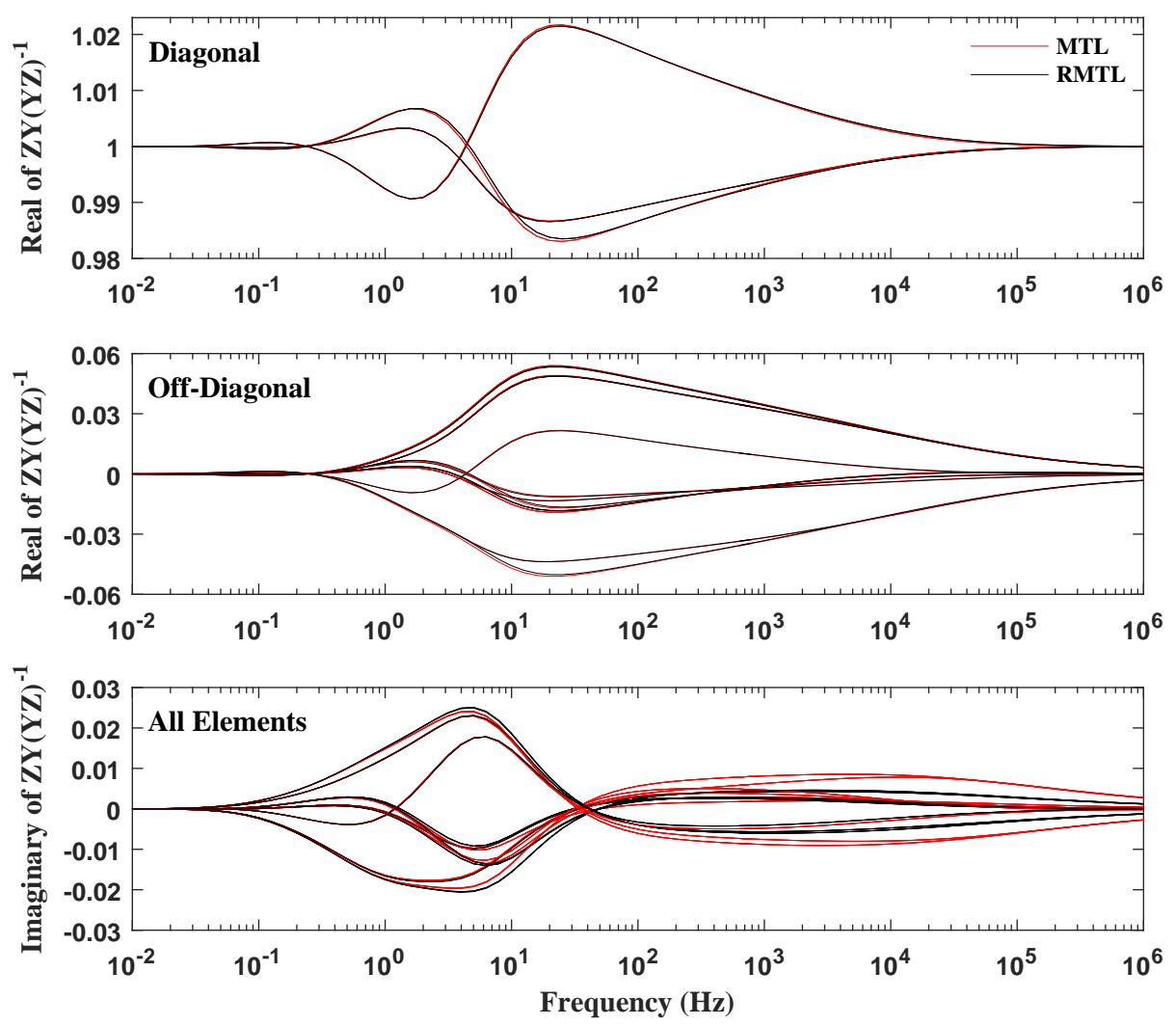

Figure 4.3: Validity of the collocation condition. If $\mathbf{Z Y}=\mathbf{Y Z}$, the product $\mathbf{Z Y}(\mathbf{Y Z})^{-1}$ should be one for the diagonals and zero for the offdiagonals.

enforce the collocation condition as exact and incorporate some corrections to the modelling.

\subsection{Diagonalization of the RMTL Equations}

As indicated in (2.22), to diagonalize the MTL equations, it is necessary to find the matrix of eigenvectors $\mathbf{T}_{V}$ that diagonalizes the product $\mathbf{Z Y}$, and the matrix of eigenvectors $\mathbf{T}_{I}$ that diagonalize the product $\mathbf{Y Z}$. In general, since it is assumed that the products $\mathbf{Z Y}$ and $\mathbf{Y Z}$ and are different and complex, $\mathbf{T}_{V}$ and $\mathbf{T}_{I}$ will also be complex and different from each other. Also, due to the frequency dependence of the elements of $\mathbf{Z}, \mathbf{T}_{V}$ and $\mathbf{T}_{I}$ will be frequency dependent. From the theory in 
[56] [128], the $\mathbf{T}_{V}$ and $\mathbf{T}_{I}$ matrices that diagonalize $\mathbf{Z Y}$ and $\mathbf{Y Z}$ will also diagonalize the individual $\mathbf{Z}$ and $\mathbf{Y}$ matrices, $\mathbf{Z}_{m}=\mathbf{T}_{V}^{-1} \mathbf{Z} \mathbf{T}_{I}$ and $\mathbf{Y}_{m}=\mathbf{T}_{I}^{-1} \mathbf{Y} \mathbf{T}_{V}$, where subscript $m$ denotes the modal diagonalized quantities. The proposed RMTL equations are based on satisfying the collocation condition (4.12) $\mathbf{Z Y}=\mathbf{Y Z}$. To achieve this, while minimizing the effect of the inconsistencies of the MTL description, we propose that instead of first diagonalizing the matrix products $\mathbf{Z Y}$ and $\mathbf{Y Z}$, as normally done, we first diagonalize the individual $\mathbf{Z}$ and $\mathbf{Y}$ matrices. We will start by finding a transformation matrix $\mathbf{T}$ that diagonalizes $\mathbf{Z}$, and then we will use $\mathbf{T}$ to diagonalize $\mathbf{Y}$ with a very good approximation. The accuracy of this approximation will be assessed in Section 4.6 and Section 4.7. This way the products $\mathbf{Z Y}$ and $\mathbf{Y Z}$ will be diagonalized by the same matrix, that is

$$
\begin{aligned}
\mathbf{Z}_{p h} \mathbf{Y}_{p h} & =\mathbf{T} \mathbf{Z}_{m} \mathbf{T}^{-1} \mathbf{T} \mathbf{Y}_{m} \mathbf{T}^{-1} \\
& =\mathbf{T} \mathbf{Z}_{m} \mathbf{Y}_{m} \mathbf{T}^{-1}=\mathbf{T} \mathbf{Y}_{m} \mathbf{Z}_{m} \mathbf{T}^{-1}=\mathbf{Y}_{p h} \mathbf{Z}_{p h}
\end{aligned}
$$

Because $\mathbf{T}$ will diagonalize the matrix of impedances, we will additionally require that $\mathbf{T}$ preserves the nature of the real and imaginary parts of $\mathbf{Z}$, that is, the losses and time constants should be the same in the original phase system and in the modal system. The same applies to $\mathbf{T}$ diagonalizing $\mathbf{Y}$. This leads to the argument that there must be a solution for $\mathbf{T}$ that is real. This argument is consistent with the property in matrix algebra that the eigenvectors that diagonalize a symmetrical positive definite real matrix have a real solution (in addition to other possible complex solutions).

\subsection{Transformation Matrix $T$ to Diagonalize $Z$ and $Y$}

Since $\mathbf{Z}$ is subjected to skin effect, while $\mathbf{Y}$ is formulated based on the constant $\mathbf{G}_{\text {ins }}$ and $\mathbf{C}_{\text {ext }}$, it is a more restricted problem to diagonalize $\mathbf{Z}$ than to diagonalize $\mathbf{Y}$. We will then first try to find a transformation matrix that diagonalizes $\mathbf{Z}$, with a good degree of accuracy and with certain desired properties, while keeping in mind that this matrix must also diagonalize $\mathbf{Y}$.

Now, suppose in (4.19) that instead of diagonalizing the full $\mathbf{Z}$ directly, we first diagonalize its dominant component $\mathbf{L}_{\text {loop }}$. Since $\mathbf{L}_{\text {loop }}$ is a symmetrical positive definite real matrix, there is a real solution for its diagonalizing matrix $\mathbf{T}$. This solution is shown in Fig. 4.4 for the line of Fig. 3.5. 

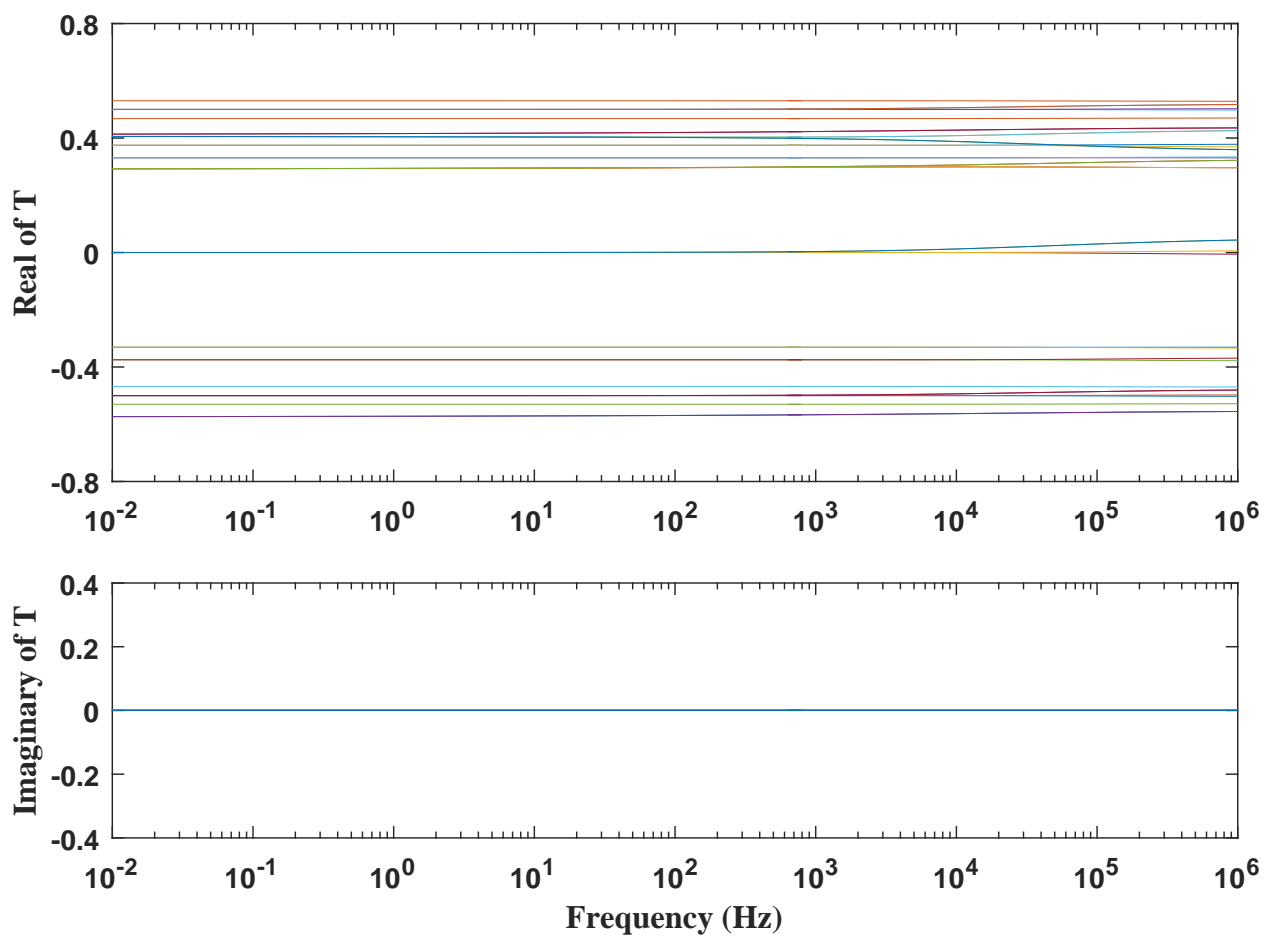

Figure 4.4: RMTL formulation. $\mathbf{T}$ matrix to diagonalize $\mathbf{L}_{\text {loop }}$ for the line of Fig. 3.5. The same matrix $\mathbf{T}$ diagonalizes $\mathbf{Z Y}$ and $\mathbf{Y Z}$.

In Fig. 4.4, we can see that $\mathbf{T}$ is basically constant up to about $1 \mathrm{kHz}$, with some variations after this frequency. The maximum variance is about $2 \times 10^{-5}$ for the frequency range between $10^{-2} \mathrm{~Hz}$ and $10^{4} \mathrm{~Hz}$, and slightly larger at $10^{-4}$ for the range from $10^{4}$ to $10^{6} \mathrm{~Hz}$. This is in contrast with the strong frequency dependence of the complex $\mathbf{T}_{V}$ and $\mathbf{T}_{I}$ matrices of the current MTL formulation. For reference, these matrices are shown in Fig. 4.5 calculated using MATLAB and using the method of [135] to avoid modes switching.

To proceed with the diagonalization of (4.19), we will take a single value of $\mathbf{T}$ real. Here we use the value at $100 \mathrm{~Hz}$ which is asymptotic in the low frequencies region. Any other frequency in this region gives basically the same results.

We now apply $\mathbf{T}^{-1}$ (.)T to each term on the right hand side of (4.19). Since $\mathbf{R}_{c}$ is already diagonal, we will get the same diagonal $\mathbf{R}_{c}$. Next, we apply $\mathbf{T}^{-1}($.)T to the matrix of all ones $[\mathbf{1}]$. Notice that a matrix of all ones is a special case of a 

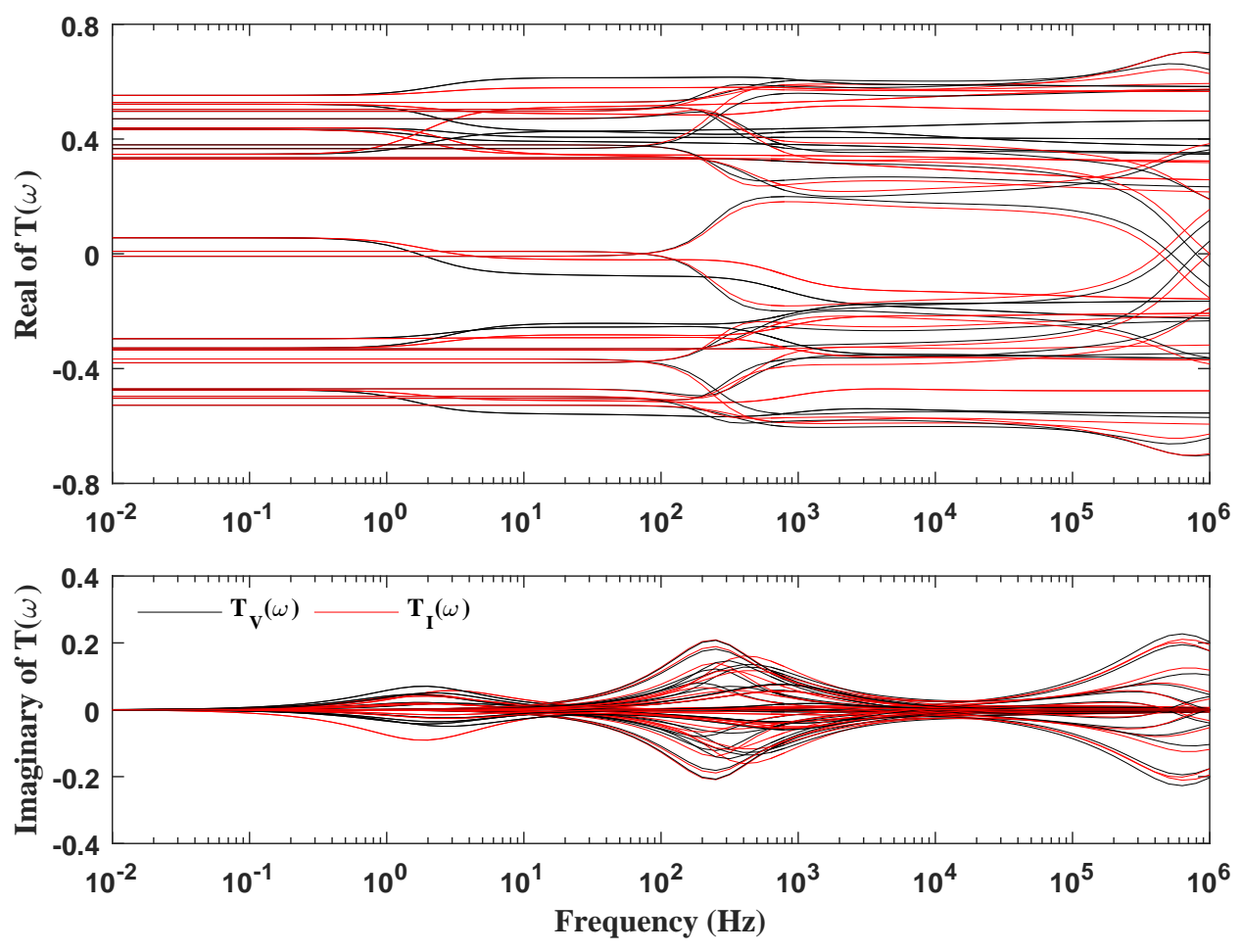

Figure 4.5: MTL formulation. Real and imaginary part of complex $\mathbf{T}_{V}$ and $\mathbf{T}_{I}$ matrices to diagonalize $\mathbf{Z Y}$ and $\mathbf{Y Z}$ for the line of Fig. 3.5.

symmetrical matrix where all the self and mutual elements are one. Using symmetrical components or other akin transformation gives, for exact diagonalization, $Z_{\text {self }}+(N-1) Z_{\text {mutual }}=N$ for the zero sequence, mode 1 , and $Z_{\text {self }}-Z_{\text {mutual }}=0$ for the line modes, modes 2 to $N$. Next, the diagonalizing matrix $\mathbf{T}$ is applied to the $\mathbf{Y}$ matrix in (4.1). Since $\mathbf{G}_{i n s}$ is already diagonal, the concern is to diagonalize the matrix of capacitances $\mathbf{C}_{e x t}$. Table 4.1 shows the errors in the diagonalization of the matrix of ones $[\mathbf{1}]$ and $\mathbf{C}_{\text {ext }}$, for the double-circuit vertical line of Fig. 3.5.

Notice that since $\mathbf{T}$ is constant, and the matrix of ones and $\mathbf{C}_{\text {ext }}$ are also constant, the errors in Table 4.1 are the same for all frequencies. As seen in this table, the diagonalization is practically exact for modes 3, 4, and 6. For the matrix of ones, the errors are $0.04 \%$ for mode $1,0.0011 \%$ for mode 2 , and $0.23 \%$ for mode 5. For $\mathbf{C}_{\text {ext }}$, the errors are $0.9 \%$ for mode $1,0.3 \%$ for mode 2 , and $0.09 \%$ for mode 5 . This accuracy has been verified for other line configurations with similar results. 
Table 4.1: Errors in the diagonalization of the matrix of ones $[\mathbf{1}]$ and $\mathbf{C}_{e x t}$ for T calculated at $100 \mathrm{~Hz}$.

\begin{tabular}{l|c|cccccc}
\hline \multicolumn{2}{c|}{ modes } & 1 & 2 & 3 & 4 & 5 & 6 \\
\hline \multirow{2}{*}[\mathbf{1}]{} & Exact & 6 & 0 & 0 & 0 & 0 & 0 \\
& Approx. & 5.9978 & $1.1 \times 10^{-5}$ & 0 & 0 & 0.0023 & 0 \\
\hline \multirow{2}{*}{$\mathbf{C}_{\text {ext }}(\mathrm{pF} / \mathrm{m})$} & Exact & 3.4574 & 7.5722 & 7.7790 & 8.9125 & 9.2466 & 9.5774 \\
& Approx. & 3.4890 & 7.5491 & 7.7792 & 8.9123 & 9.2380 & 9.5773 \\
\hline
\end{tabular}

The results shown in Table 4.1 justify the validity of diagonalizing $\mathbf{Z}$ and $\mathbf{Y}$ separately with the same matrix $\mathbf{T}$. Next we look at the capability of $\mathbf{T}$ to diagonalize the products $\mathbf{Z Y}$ and $\mathbf{Y Z}$.

\subsection{Transformation Matrix T to Diagonalize $\mathrm{ZY}$ and $\mathrm{YZ}$}

The premise in obtaining a single real constant $\mathbf{T}$ was that this matrix would be able to diagonalize both $\mathbf{Z Y}$ for the propagation of the voltages and $\mathbf{Y Z}$ for the propagation of the currents, and thus satisfy the collocation condition (4.12).

In the plots of Fig. 4.6 we show the magnitude of the off-diagonal elements after diagonalization. Ideally, these elements should be zero. To show the relative errors, the values were divided by the geometrical average of the corresponding diagonal elements at each frequency. The largest errors occur between 10 and 100 $\mathrm{Hz}$ for the off-diagonal elements $(5,6)$ and $(2,6)$. However, since in the line model the off-diagonals are made exactly zero, these errors are not significant as long as the values of the diagonals are accurate.

In 4.2 and 4.3 , we assess the accuracy of the diagonal elements resulting from the diagonalization of $\mathbf{Z Y}$ using the proposed $\mathbf{T}$ matrix versus the traditional $\mathbf{T}_{V}$ matrix. For each frequency, the first row corresponds to $\mathbf{T}$ and the second row to $\mathbf{T}_{V}$. The accuracy is similar for the diagonalization of the propagation of the currents (not shown). The numbers in these tables have been divided by the speed of light. Even though all numbers are negative, they are shown as positive in the tables to save space.

Notice that the real parts (4.2) are one to two orders of magnitudes larger than the imaginary parts $(4.3)$. The errors in the real parts are less than $0.1 \%$ for all 

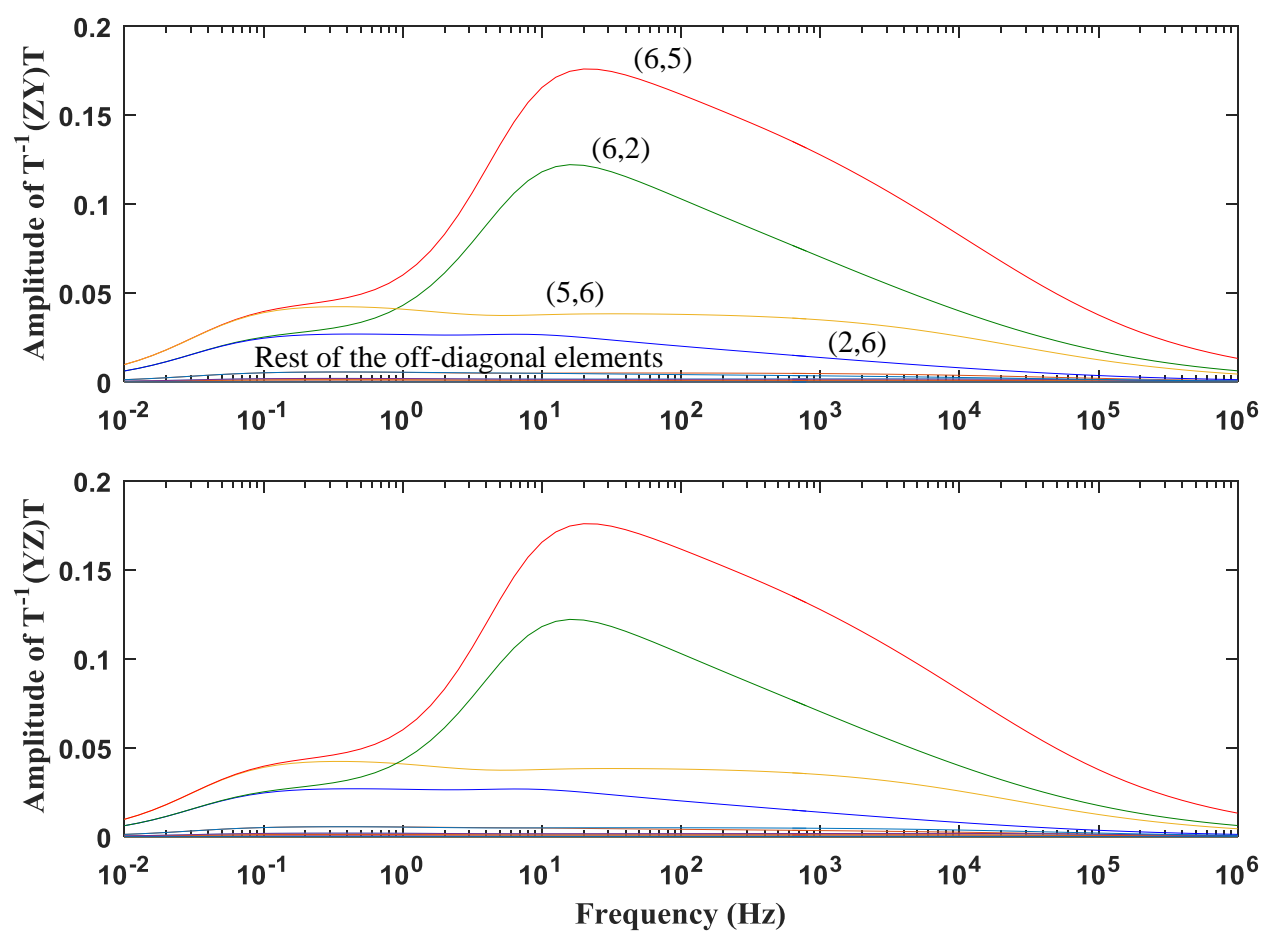

Figure 4.6: Diagonalization of $\mathbf{T}^{-1} \mathbf{Z Y T}$ and $\mathbf{T}^{-1} \mathbf{Y Z T}$. The plots show the absolute value of the off-diagonal elements.

modes, except at very low frequencies where the magnitudes are very small. The errors for the imaginary parts remain less than $1.5 \%$ for all modes for frequencies below $100 \mathrm{kHz}$ (which is the normal range for switching transients in power systems), while they grow up to $4 \%$ for the ground mode, and higher for some of the line modes, for higher frequencies. For frequencies above $1 \mathrm{MHz}$, the basic assumptions of TEM propagation become questionable [124]. 
Table 4.2: Real part of the eigenvalues of $\mathbf{Z Y}$ calculated using the proposed single $\mathbf{T}$ versus exact diagonalization (all values are negative).

\begin{tabular}{c|cccccc}
\hline$f$ & Mode 1 & Mode 2 & Mode 3 & Mode 4 & Mode 5 & Mode 6 \\
\hline \hline \multirow{2}{*}{$1 \mathrm{~Hz}$} & $3.418 \mathrm{e}-7$ & $1.026 \mathrm{e}-7$ & $1.017 \mathrm{e}-7$ & $1.016 \mathrm{e}-7$ & $1.022 \mathrm{e}-7$ & $1.019 \mathrm{e}-7$ \\
& $3.370 \mathrm{e}-7$ & $1.067 \mathrm{e}-7$ & $1.017 \mathrm{e}-7$ & $1.019 \mathrm{e}-7$ & $1.016 \mathrm{e}-7$ & $1.038 \mathrm{e}-7$ \\
\hline \multirow{2}{*}{$100 \mathrm{~Hz}$} & $2.685 \mathrm{e}-3$ & $1.378 \mathrm{e}-3$ & $1.369 \mathrm{e}-3$ & $1.367 \mathrm{e}-3$ & $1.372 \mathrm{e}-3$ & $1.370 \mathrm{e}-3$ \\
& $2.699 \mathrm{e}-3$ & $1.367 \mathrm{e}-3$ & $1.369 \mathrm{e}-3$ & $1.370 \mathrm{e}-3$ & $1.367 \mathrm{e}-3$ & $1.368 \mathrm{e}-3$ \\
\hline \multirow{2}{*}{$1 \mathrm{kHz}$} & $2.155 \mathrm{e}-1$ & $1.354 \mathrm{e}-1$ & $1.345 \mathrm{e}-1$ & $1.339 \mathrm{e}-1$ & $1.342 \mathrm{e}-1$ & $1.340 \mathrm{e}-1$ \\
& $2.168 \mathrm{e}-1$ & $1.343 \mathrm{e}-1$ & $1.346 \mathrm{e}-1$ & $1.340 \mathrm{e}-1$ & $1.339 \mathrm{e}-1$ & $1.339 \mathrm{e}-1$ \\
\hline \multirow{2}{*}{$10 \mathrm{~Hz}$} & $1.725 \mathrm{e}+1$ & $1.338 \mathrm{e}+1$ & $1.331 \mathrm{e}+1$ & $1.324 \mathrm{e}+1$ & $1.325 \mathrm{e}+1$ & $1.324 \mathrm{e}+1$ \\
& $1.736 \mathrm{e}+1$ & $1.328 \mathrm{e}+1$ & $1.332 \mathrm{e}+1$ & $1.324 \mathrm{e}+1$ & $1.323 \mathrm{e}+1$ & $1.323 \mathrm{e}+1$ \\
\hline \multirow{2}{*}{$100 \mathrm{~Hz}$} & $1.471 \mathrm{e}+3$ & $1.327 \mathrm{e}+3$ & $1.323 \mathrm{e}+3$ & $1.319 \mathrm{e}+3$ & $1.319 \mathrm{e}+3$ & $1.319 \mathrm{e}+3$ \\
& $1.477 \mathrm{e}+3$ & $1.321 \mathrm{e}+3$ & $1.324 \mathrm{e}+3$ & $1.318 \mathrm{e}+3$ & $1.318 \mathrm{e}+3$ & $1.318 \mathrm{e}+3$ \\
\hline \multirow{2}{*}{$1 \mathrm{MHz}$} & $1.367 \mathrm{e}+5$ & $1.320 \mathrm{e}+5$ & $1.319 \mathrm{e}+5$ & $1.317 \mathrm{e}+5$ & $1.317 \mathrm{e}+5$ & $1.317 \mathrm{e}+5$ \\
& $1.369 \mathrm{e}+5$ & $1.328 \mathrm{e}+5$ & $1.319 \mathrm{e}+5$ & $1.317 \mathrm{e}+5$ & $1.317 \mathrm{e}+5$ & $1.317 \mathrm{e}+5$ \\
\hline
\end{tabular}

Table 4.3: Imaginary part of the eigenvalues of $\mathbf{Z Y}$ calculated using the proposed single $\mathbf{T}$ versus exact diagonalization (all values are negative).

\begin{tabular}{c|cccccc}
\hline$f$ & Mode 1 & Mode 2 & Mode 3 & Mode 4 & Mode 5 & Mode 6 \\
\hline \hline \multirow{2}{*}{$1 \mathrm{~Hz}$} & $4.615 \mathrm{e}-7$ & $8.454 \mathrm{e}-7$ & $8.708 \mathrm{e}-7$ & $9.961 \mathrm{e}-7$ & $1.032 \mathrm{e}-6$ & $1.070 \mathrm{e}-6$ \\
& $4.607 \mathrm{e}-7$ & $8.458 \mathrm{e}-7$ & $8.707 \mathrm{e}-7$ & $9.961 \mathrm{e}-7$ & $1.070 \mathrm{e}-6$ & $1.033 \mathrm{e}-6$ \\
\hline \multirow{2}{*}{$100 \mathrm{~Hz}$} & $4.110 \mathrm{e}-4$ & $9.195 \mathrm{e}-5$ & $9.468 \mathrm{e}-5$ & $1.083 \mathrm{e}-4$ & $1.130 \mathrm{e}-4$ & $1.163 \mathrm{e}-4$ \\
& $4.131 \mathrm{e}-4$ & $9.169 \mathrm{e}-5$ & $9.528 \mathrm{e}-5$ & $1.079 \mathrm{e}-4$ & $1.162 \mathrm{e}-4$ & $1.117 \mathrm{e}-4$ \\
\hline \multirow{2}{*}{$1 \mathrm{kHz}$} & $3.331 \mathrm{e}-2$ & $2.174 \mathrm{e}-3$ & $2.235 \mathrm{e}-3$ & $2.560 \mathrm{e}-3$ & $2.720 \mathrm{e}-3$ & $2.750 \mathrm{e}-3$ \\
& $3.348 \mathrm{e}-2$ & $2.260 \mathrm{e}-3$ & $2.325 \mathrm{e}-3$ & $2.522 \mathrm{e}-3$ & $2.730 \mathrm{e}-3$ & $2.632 \mathrm{e}-3$ \\
\hline \multirow{2}{*}{$10 \mathrm{~Hz}$} & $2.341 \mathrm{e} 0$ & $6.377 \mathrm{e}-2$ & $6.540 \mathrm{e}-2$ & $7.491 \mathrm{e}-2$ & $8.245 \mathrm{e}-2$ & $8.050 \mathrm{e}-2$ \\
& $2.377 \mathrm{e} 0$ & $7.760 \mathrm{e}-2$ & $8.113 \mathrm{e}-2$ & $7.443 \mathrm{e}-2$ & $8.007 \mathrm{e}-2$ & $7.713 \mathrm{e}-2$ \\
\hline \multirow{2}{*}{$100 \mathrm{~Hz}$} & $1.191 \mathrm{e}+2$ & $1.974 \mathrm{e} 0$ & $2.019 \mathrm{e} 0$ & $2.313 \mathrm{e} 0$ & $2.643 \mathrm{e} 0$ & $2.485 \mathrm{e} 0$ \\
& $1.231 \mathrm{e}+2$ & $3.626 \mathrm{e} 0$ & $4.586 \mathrm{e} 0$ & $2.339 \mathrm{e} 0$ & $2.472 \mathrm{e} 0$ & $2.386 \mathrm{e} 0$ \\
\hline \multirow{2}{*}{$1 \mathrm{MHz}$} & $4.590 \mathrm{e}+3$ & $0.621 \mathrm{e}+2$ & $0.634 \mathrm{e}+2$ & $7.258 \mathrm{e}+1$ & $8.471 \mathrm{e}+1$ & $7.800 \mathrm{e}+1$ \\
& $4.806 \mathrm{e}+3$ & $1.571 \mathrm{e}+2$ & $2.304 \mathrm{e}+2$ & $7.592 \mathrm{e}+1$ & $7.772 \mathrm{e}+1$ & $7.513 \mathrm{e}+1$ \\
\hline
\end{tabular}

\subsection{Proposed Frequency-Dependent Line Model}

The wave collocation conditions (4.11) (4.12) result in simpler RMTL wave propagation equations. For convenience, we repeat (4.12) here, 


$$
\mathbf{Z Y}=\mathbf{Y Z}
$$

With this condition, (2.15) (2.16) become

$$
\begin{gathered}
\mathbf{V}(x)=\mathbf{V}_{f k} e^{-\gamma x}+\mathbf{V}_{b k} e^{+\gamma x} \\
\mathbf{I}(x)=\mathbf{I}_{f k} e^{-\gamma x}+\mathbf{I}_{b k} e^{+\gamma x}
\end{gathered}
$$

where the same propagation constant applies to both, voltages and currents,

$$
\gamma=\sqrt{\mathbf{Z Y}}=\sqrt{\mathbf{Y Z}}
$$

The voltage and current waves are related by

$$
\mathbf{V}(x)=\mathbf{V}_{f k} e^{-\gamma x}+\mathbf{V}_{b k} e^{+\gamma x}=\mathbf{Z}_{c}\left(\mathbf{I}_{f k} e^{-\gamma x}+\mathbf{I}_{b k} e^{+\gamma x}\right)
$$

where the characteristic impedance is given by (4.9) and repeated here,

$$
\mathbf{Z}_{c}=\mathbf{Y}^{-1} \sqrt{\mathbf{Y Z}}
$$

Equations (4.22) to (4.27) constitute the RMTL and will be used to formulate the proposed FDLM model.

Combining (4.23) (4.24) as in [69], we obtain the forward perturbation wave

$$
\mathbf{V}(x)+\mathbf{Z}_{c} \mathbf{I}(x)=\left(\mathbf{V}_{k}+\mathbf{Z}_{c} \mathbf{I}_{k}\right) e^{-\gamma x}
$$

Note that in the existing FDLINE model of [69], (4.28) can only be written in terms of individual decoupled modes (scalar quantities), while in FDLM, because of having a single $\gamma,(4.28)$ applies also to the full phase-coordinates matrices.

Equation (4.28) leads to the equivalent circuit of Fig. 4.7. With $x=0$ for the sending end $k$ of the line, and $x=\ell$ for the receiving end $n$, one can write,

$$
\mathbf{V}_{n}+\mathbf{Z}_{c} \mathbf{I}_{n}=\left(\mathbf{V}_{k}+\mathbf{Z}_{c} \mathbf{I}_{k}\right) e^{-\gamma \ell}
$$

The circuit of Fig. 4.7-right results from (4.29) reversing the direction of the current. A similar analysis can be followed to obtain the circuit of Fig. 4.7-left for 


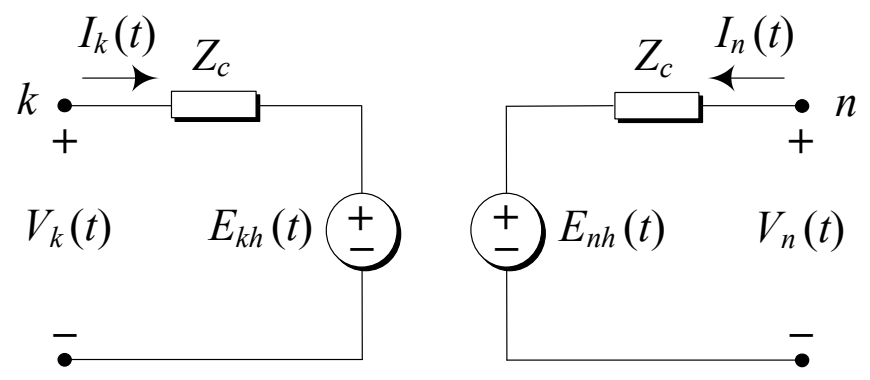

Figure 4.7: FDLM equivalent circuit in the frequency domain. Quantities can be matrices in the phase domain or scalar quantities in the modal domain.

the line as seen from node $k$. The history sources in Fig. 4.7 are given by

$$
\mathbf{E}_{n h}=\left(\mathbf{V}_{k}+\mathbf{Z}_{c} \mathbf{I}_{k}\right) e^{-\gamma \ell} \quad, \quad \mathbf{E}_{k h}=\left(\mathbf{V}_{n}+\mathbf{Z}_{c} \mathbf{I}_{n}\right) e^{-\gamma \ell}
$$

For multiconductor lines in phase coordinates, the quantities in Fig. 4.7 are matrices. For decoupled modal coordinates, the quantities are scalars for each propagation mode.

The modal (diagonal) series impedance and shunt admittance matrices are given by

$$
\mathbf{Z}_{m}=\mathbf{T}^{-1} \mathbf{Z}_{p h} \mathbf{T} \quad, \quad \mathbf{Y}_{m}=\mathbf{T}^{-1} \mathbf{Y}_{p h} \mathbf{T}
$$

where now in FDLM we have a single real frequency-independent transformation matrix $\mathbf{T}$ in (4.31) instead of two complex frequency dependent transformation matrices $\mathbf{T}_{V}$ and $\mathbf{T}_{I}$ in (2.23).

The modal characteristic impedance $Z_{c m}$ and the modal propagation function $\gamma_{m}$ are calculated at each frequency from

$$
Z_{c m}=\sqrt{\frac{Z_{m}}{Y_{m}}} \quad, \quad \gamma_{m}=\sqrt{Z_{m} Y_{m}}
$$

In FDLM, like in FDLINE, the modal frequency dependent characteristic impedance and propagation functions are synthesized using negative poles and zeroes for a passive realization. The improved BAF of [1] is used for the synthesis. For the line of Fig. 3.5, the maximum errors with BAF were less than $0.1 \%$ for the propagation functions and less than $0.5 \%$ for the characteristic impedance functions over the entire frequency range. 


\subsection{Numerical Results: Transient Simulations}

This section compares transient simulation results obtained with the proposed FDLM line model and the traditional EMTP FDLINE and ULM formulations. In previous work in [116], we showed that FDLINE and ULM gave very similar accuracy for asymmetrical overhead line configurations.

The analysis in the present section further makes the case for the validity of using a single transformation matrix. Here, the proposed FDLM model is compared to FDLINE and ULM for the double circuit vertical line of Fig. 3.5 [34], which corresponds to the structure that resulted in higher errors in [116].

To validate the representation of the transformation matrices, a very accurate DTFS solution was implemented as proposed in Chapter 3.

Simulation tools: PSCAD v4.5.2 for ULM, Microtran v3.25 for FDLINE, and MATLAB for FDLM and DTFS.

All methods: simulation time of interest $=50 \mathrm{~ms}$, time step $\Delta t=50 \mu \mathrm{s}$.

DTFS: sampling frequency $f_{c}=20 \mathrm{kHz}$, time window width $T_{c}=3 \mathrm{~s}$.

Line models: The number of poles for the fitting of the line functions was set to a maximum of 35 for FDLINE, ULM, and FDLM. An average of about 10 poles per function was used by the models. The frequency range was from $10^{-2}$ to $10^{7}$ Hz. For fDline, $\mathbf{T}_{V}$ and $\mathbf{T}_{I}$ were calculated at $1 \mathrm{kHz}$. For FDLM, $\mathbf{T}$ was calculated at $100 \mathrm{~Hz}$.

Figures 4.8 and 4.9 show the results for the open circuit voltage $v_{3}$ and the short circuit current $i_{6}$ for the test of Fig. 3.6 using FDLM, FDLINE, and ULM for the time domain solutions and DTFS for the frequency domain solutions. FDLINE and ULM use the MTL equations while FDLM uses the RMTL equations. Two solutions are calculated with DTFS: a) For the MTL equations, calculating $\mathbf{T}_{V}$ and $\mathbf{T}_{I}$ at each frequency, and b) For the RMTL equations, calculating $\mathbf{T}$ at each frequency.

Figure 4.10 shows the instantaneous errors over the time line of the simulation until steady state is reached. In general the errors for the currents were smaller than the errors for the voltages.

In Fig. 4.9 and Fig. 4.10 for $v_{3}$, we can observe that all curves are basically superimposed around the first (negative) peak. On the second (positive) peak, ULM 

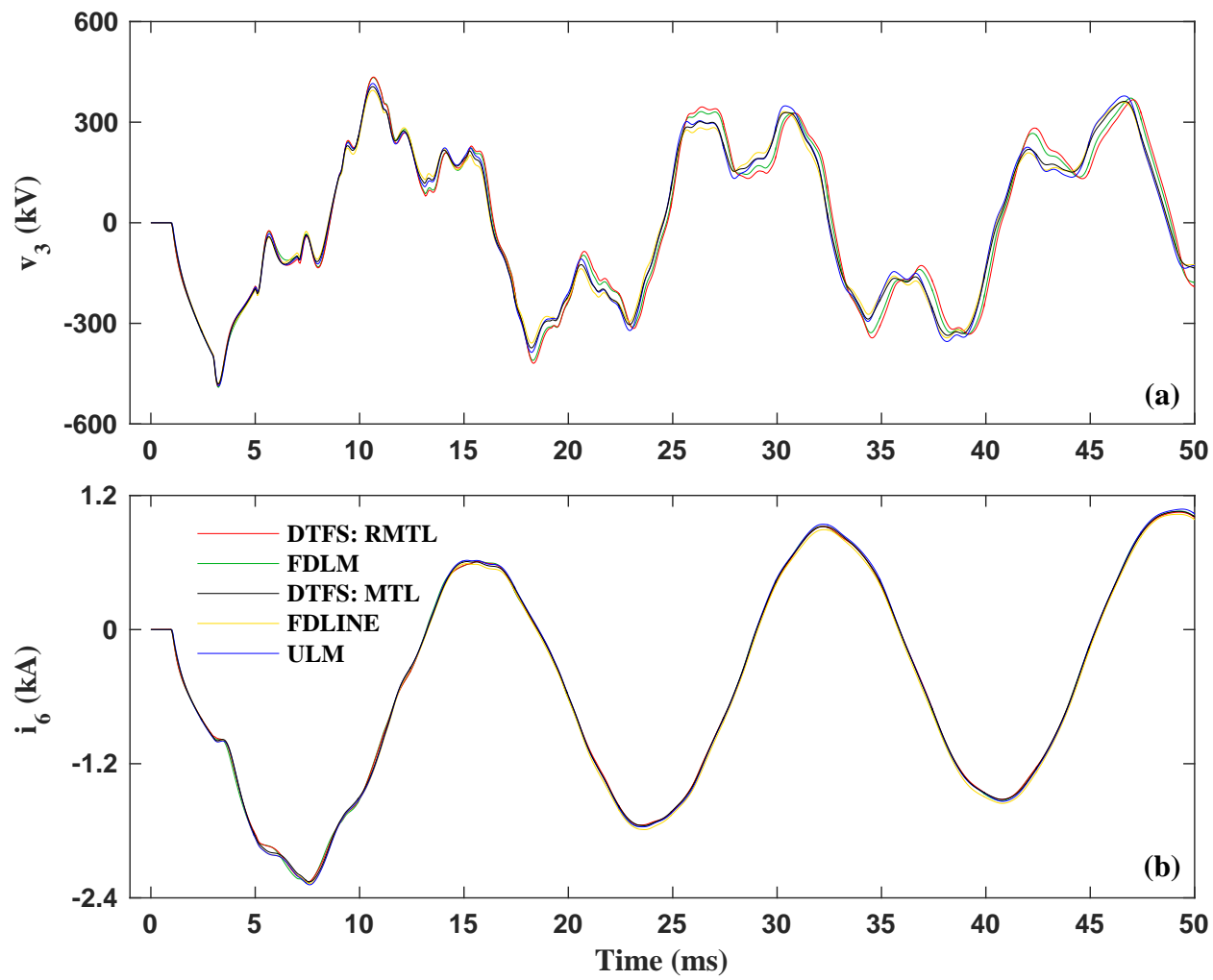

Figure 4.8: Comparison of MTL (FDLINE, ULM) and RMTL (FDLM) line models for the unbalanced fault test of Fig. 3.6.

is slightly closer to the reference solution than FDLINE, but the errors for ULM increase in the subsequent cycles. This is shown more clearly in Fig. 4.10. This is probably related to the less accurate determination by ULM of the propagation delays.

The errors for FDLM remain relatively low over the entire simulation time window. It can be seen in Fig. 4.8 that there is a slight phase shift between the MTL and the RMTL solutions that increases as time advances. This shift might be related to the waves collocation issues discussed in this chapter. All simulations simulations converge when steady state is reached.

Table 4.4 shows the maximum instantaneous errors for all voltages and currents. The maximum relative errors for FDLINE and FDLM remained relatively 

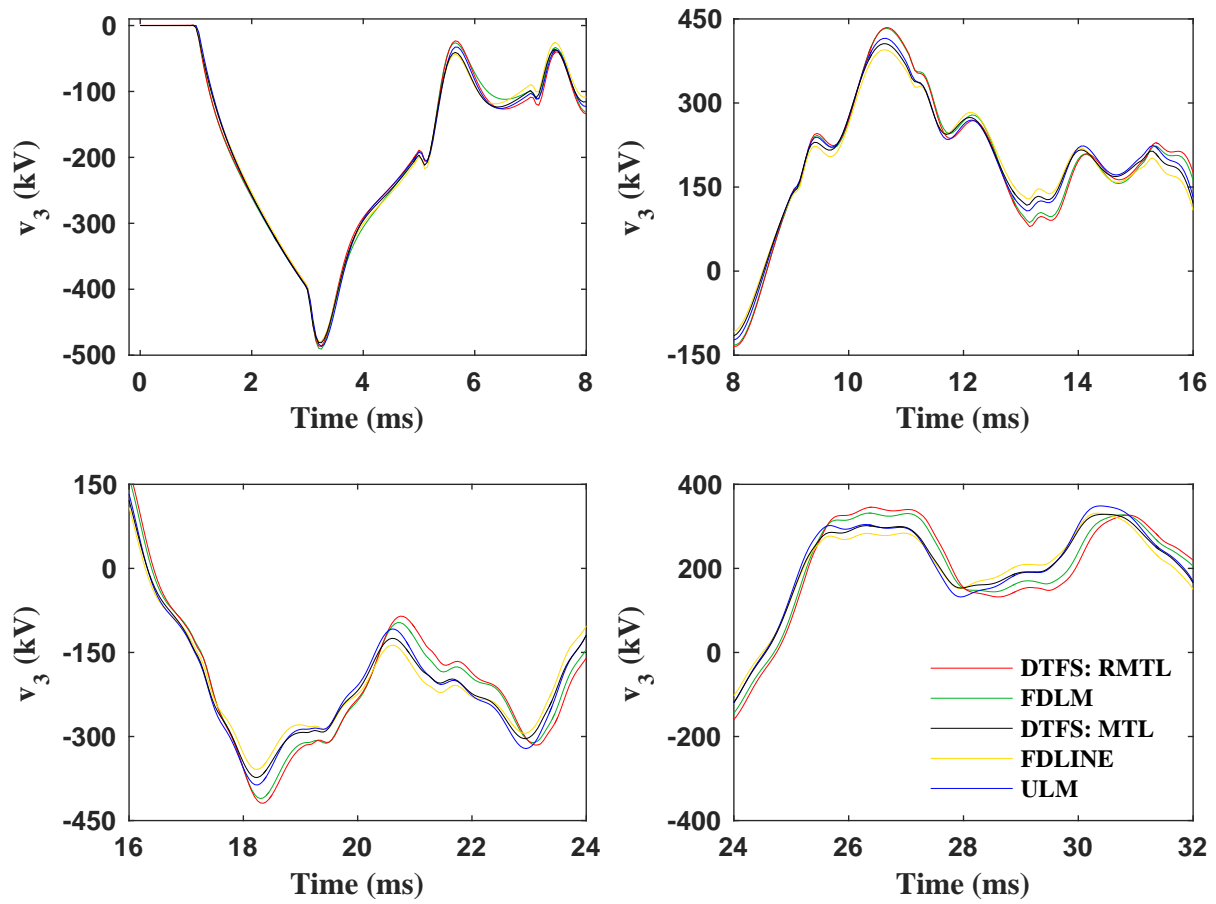

Figure 4.9: Details of the first four peaks of $v_{3}$ in Fig. 4.8a.

even, while the maximum errors were larger for ULM for $v_{1}$ and $v_{4}$. The results for FDLINE and ULM were consistent with the previous results of [116].

Table 4.4: Maximum errors for voltages and currents for FDLINE, ULM, and FDLM with respect to their reference DTFS solution.

\begin{tabular}{c|ccc}
\hline$f$ & FDLINE & ULM & FDLM \\
\hline \hline$v_{1}$ & $4.26 \%$ & $10.50 \%$ & $5.07 \%$ \\
$v_{3}$ & $7.18 \%$ & $8.56 \%$ & $6.67 \%$ \\
$v_{4}$ & $5.53 \%$ & $10.48 \%$ & $5.03 \%$ \\
$v_{5}$ & $8.19 \%$ & $12.15 \%$ & $5.42 \%$ \\
$i_{2}$ & $2.30 \%$ & $2.33 \%$ & $2.07 \%$ \\
$i_{6}$ & $3.10 \%$ & $2.13 \%$ & $2.04 \%$ \\
\hline
\end{tabular}




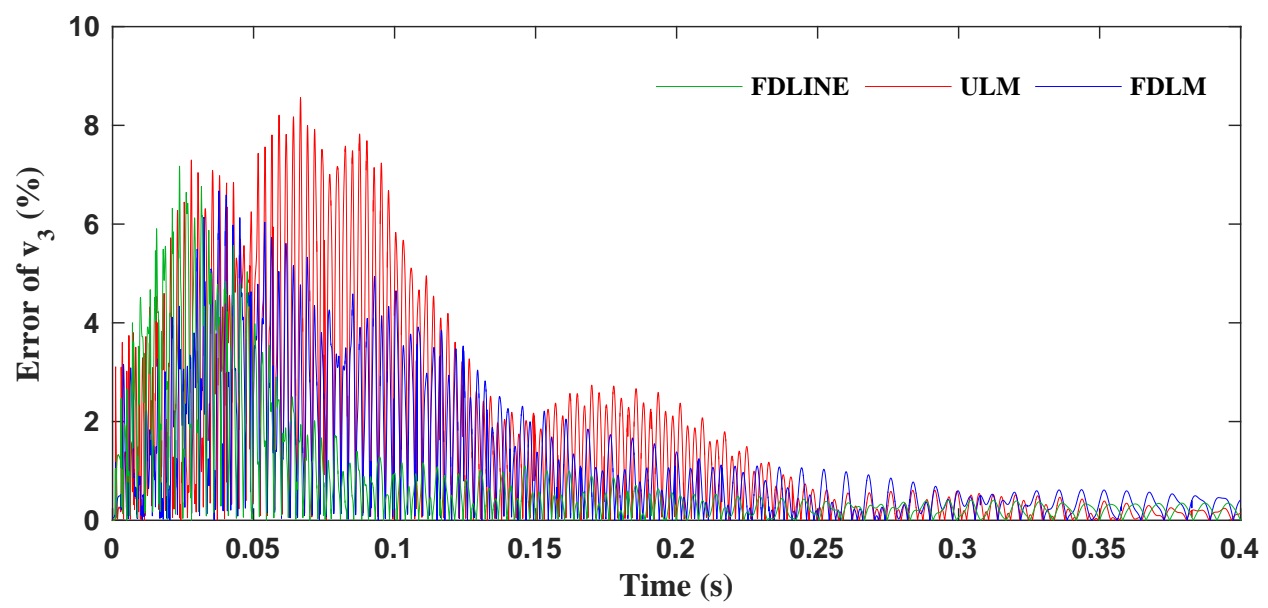

Figure 4.10: Errors for the voltage $v_{3}$ for FDLINE and ULM versus DTFS for the MTL equations and for FDLM versus DTFS for the RMTL equations.

Computational efficiency of FDLINE, ULM, and FDLM are compared in Table 4.5 in the simulation of unbalanced faults test of Fig. 3.6. For this comparison, operations count per time step is calculated for FDLINE and FDLM using (2.31) and for ULM using (2.35). The fitting information were extracted from Microtran for FDLINE, PSCAD for ULM, and BAF algorithm of [1] for FDLM.

Table 4.5: Comparison of operations count for FDLINE, ULM, and FDLM line models in the simulation of unbalanced faults test of Fig. 3.6.

\begin{tabular}{c|ccc}
\hline Models & $Z_{c}$ or $Y_{c}$ & $e^{-\gamma \ell}$ & total \\
\hline \hline FDLINE & $6 \times 6+142$ & $6 \times 6+182$ & 396 \\
FDLM & $6 \times 6+87$ & $6 \times 6+231$ & 390 \\
ULM & $6 \times 6 \times 11$ & $6 \times 6 \times 41$ & 1872 \\
\hline
\end{tabular}

As indicated in Table 4.5, ULM is about 4.7 times more expensive than FDLINE and FDLM in terms of computational cost. Even though, ULM requires fewer poles for fitting compared to FDLINE and FDLM, the main computational burden is due to fitting the frequency dependence of the transformation matrices. Since FDLINE and FDLM use the same fitting routine, their computational cost are relatively similar. 
Figure 4.11 show the results for the open circuit voltage $v_{1}$ for the test of Fig. 3.8 obtained with FDLM with $\mathbf{T}$ calculated at $1 \mathrm{~Hz}, 100 \mathrm{~Hz}$, and $1 \mathrm{kHz}$ compared to the DTFS with $\mathbf{T}$ calculated at each frequency.

As opposed to the results of Fig. 3.30 where the accuracy of FDLINE depended on the chosen frequency, FDLM is a frequency-independent model.
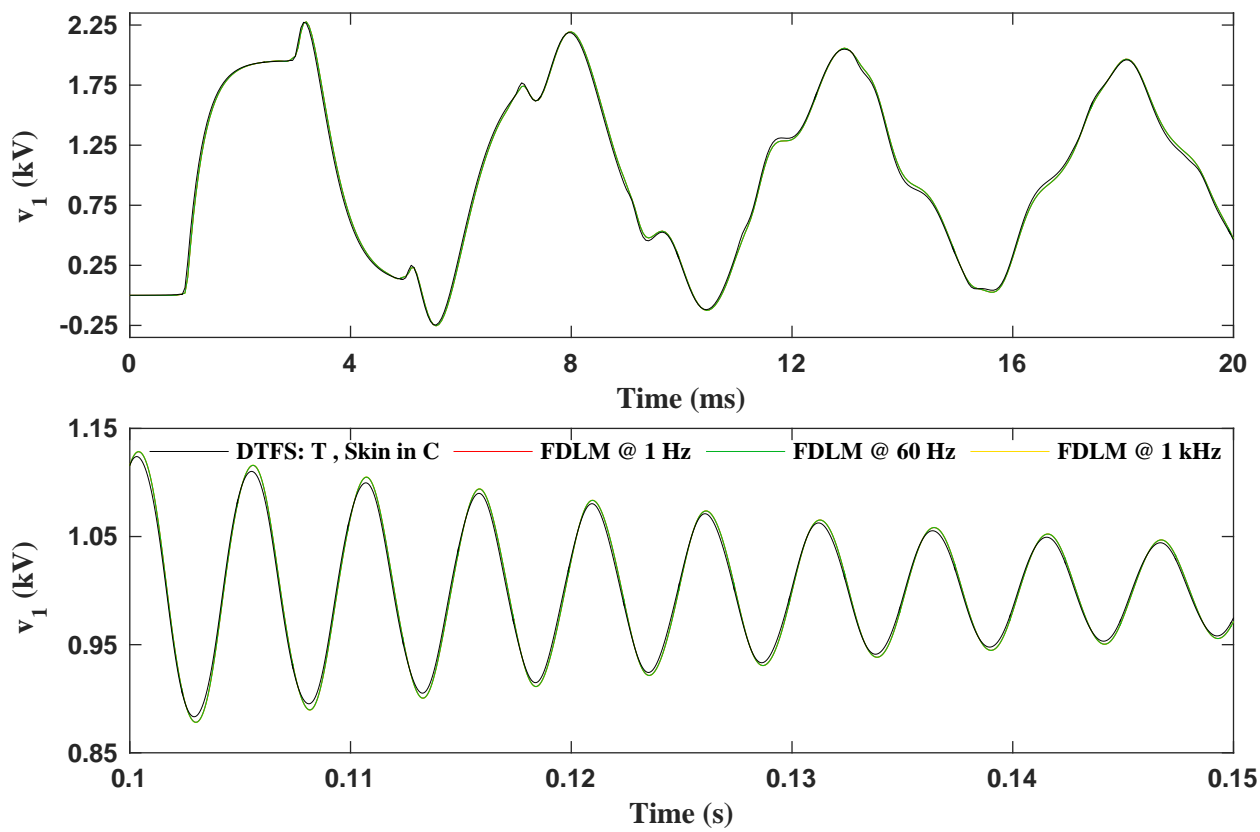

Figure 4.11: $v_{1}$ in the step response test of Fig. 3.8. Comparison of RMTL models: DTFS versus FDLM (for FDLM $\mathbf{T}$ calculated at different frequencies).

\subsection{General Observations}

The fundamental constraint for the MTL equations proposed in this chapter was that conceptually the voltage and current waves must be collocated and travel together with the same propagation function. Another factor for maintaining the physical consistency in the line equations is to make the ground resistance contributions to all elements of the $\mathbf{Z}$ matrix equal at each frequency. Based on this condition, the RMTL equations are proposed. 
The diagonalization method to find real transformation matrices for multiconductor transmission lines described by the RMTL equations maintains the geometrical identity of the propagation modes by separating the inductance and capacitance (responsible for propagation) from the losses. The problem is then reduced to diagonalizing a real matrix $\mathbf{L}_{\text {loop }}$, which can be achieved with a simple eigenvectors routine. There are no mode switchings in this process. This process results in a real transformation matrix that is practically constant over the entire frequency range. Error analysis demonstrated the validity of this assumption for diagonalization of the individual $\mathbf{Z}$ and $\mathbf{Y}$ and the product $\mathbf{Z Y}$ and $\mathbf{Y Z}$ for an asymmetrical line configuration.

The fact that it is possible to find a real constant transformation matrix to accurately diagonalize the line equations validates the physical assumption that these matrices should be diagonalized using a real transformation matrix. It also explains why the FDLINE model of [69] which uses real constant $\mathbf{T}_{V}$ and $\mathbf{T}_{I}$ as an approximation under the MTL equations, gives very accurate results, even for asymmetrical line configurations [116].

A new FDLM transmission line model was proposed in this chapter to implement the RMTL equations. For the proposed FDLM under the RMTL equations, using a real constant $\mathbf{T}$ for both voltages and currents is not an approximation but conceptually exact, within the limits of validity of these equations.

Simulation results were used to compare the EMTP frequency dependent line models FDLINE and ULM, and the proposed FDLM model, against reference frequency domain solutions using the MTL and the RMTL equations. The simulation results were very close for all models, with smaller overall errors for FDLM, followed by FDLINE. Overall, the results obtained with the proposed RMTL model are very close to the solutions given by the classical MTL models. These results are exact within the first cycle of the transients which the most important cycle for the setting of relays and protection devices.

More fundamental analytical work is needed to incorporate additional symmetry considerations into the line equations. One of these considerations is the principle of collocation of charge and current, which would require the re-collocation of the charge in the conductors and the ground when the current position is affected by the skin effect. In the next section, we investigate this concept with the available 
line equations.

\subsection{Further Investigations on the Wave Collocation Condition}

The waves collocation condition of (4.12) leads naturally to the concept that the external inductance for the wave propagation $\mathbf{L}_{\text {loop }}$ and the external capacitance for the wave propagation $\mathbf{C}_{\text {ext }}$ should be symmetrical. However, while $\mathbf{L}_{\text {loop }}$ is subjected to skin effect, $\mathbf{C}_{\text {ext }}$ is held constant at its electrostatic value by the fundamental assumption of TEM propagation. This inconsistency, which violates the principle of collocation of charge and current, has also an influence on the collocation of the voltage and current waves. In this chapter, we enforced the collocation of the voltage and current waves by requiring that $\mathbf{Z Y}=\mathbf{Y Z}$ (wave collocation) but more research is needed to understand how the propagation equations can be made consistent with the additional physical requirement of collocation of charge and current.

Skin effect in the capacitance has been described in the literature by as early as Wise [138], and later by Nakagawa [81] and other authors, however, it has not been incorporated in the EMTP line models.

In this section, we explore the effect of considering the "skin effect" in the capacitances on the RMTL equations proposed in this research knowing that we may contradict the TEM condition.

For consistency between the inductance and capacitance matrices, we use the Lord Kelvin's method of imaging described in Fig. 2.2 to calculate the self and mutual elements of the complex loop Maxwell's potential coefficient matrix $\mathbf{P}_{\text {loop }}^{\prime}$,

$$
P_{\text {loop } 11}^{\prime}=\frac{1}{2 \pi \varepsilon} \ln \frac{2\left(h_{1}+\bar{p}\right)}{r_{1 e q}} \quad, \quad P_{\text {loop } 12}^{\prime}=\frac{1}{2 \pi \varepsilon} \ln \frac{D_{12^{\prime}}}{d_{12}}
$$

Similarly to $\mathbf{L}_{\text {loop }}^{\prime}$, the elements of $\mathbf{C}_{\text {loop }}^{\prime}$ comprise two parts: real and imaginary. The imaginary part in (4.33) will correspond to the transversal losses due to the ground resistivity. The procedure to calculate the transversal losses are similar to the expressions (4.14). In Fig. 4.12, elements of earth transversal losses for the line of Fig. 3.5 are shown for a frequency range from $10^{-2} \mathrm{~Hz}$ to $10^{6} \mathrm{~Hz}$. 


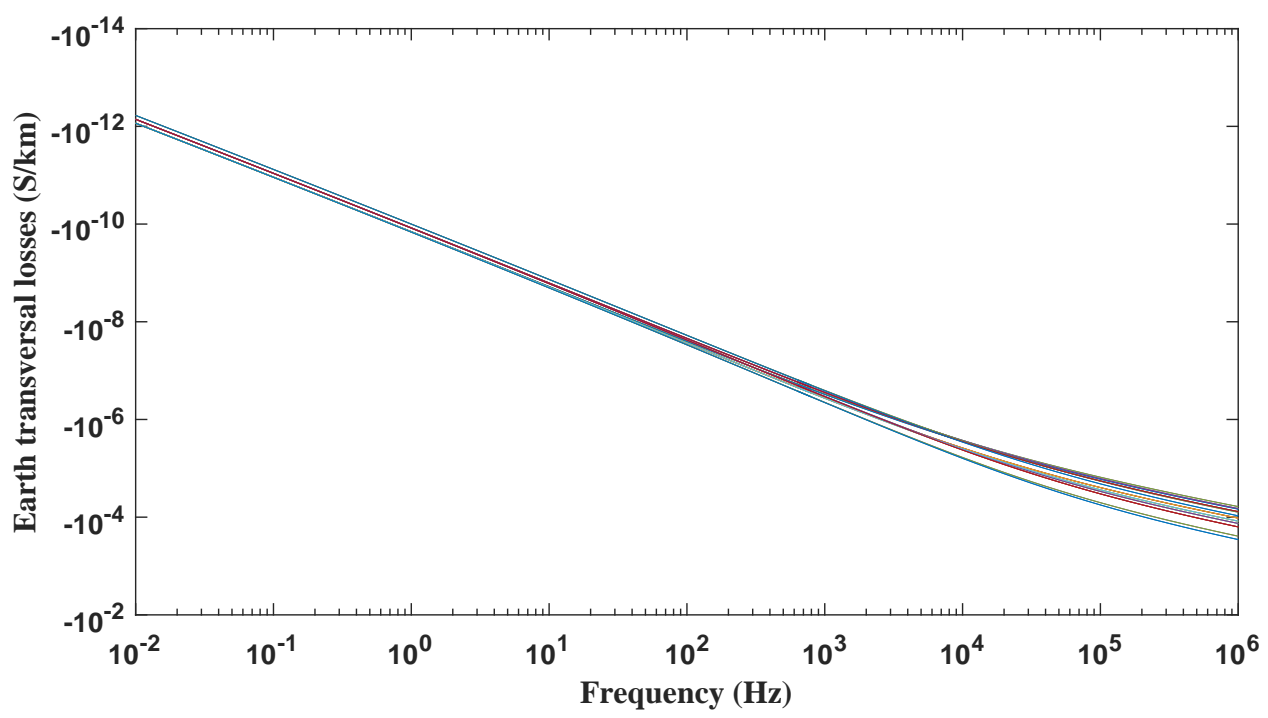

Figure 4.12: Earth transversal losses given by the complex penetration depth formula for the double circuit vertical line of Fig. 3.5

As observed in Fig. 4.12, earth transversal losses are very small quantities with negative values, therefore, to maintain the passivity in the system these elements are eliminated from the formulation.

The matrix of capacitances $\mathbf{C}_{\text {loop }}$ is obtained by taking the inverse of the real part of $\mathbf{P}_{\text {loop }}^{\prime}$,

$$
\mathbf{C}_{\text {loop }}=\mathbf{P}_{\text {loop }}^{-1}
$$

Figure 4.13 shows the elements of $\mathbf{L}_{\text {loop }}$ (valid for MTL and RMTL equations) in comparison with the elements of $\mathbf{C}_{\text {loop }}$ (investigated in this section).

As observed in Fig. 4.13 , in a frequency range of $10^{-2} \mathrm{~Hz}$ to $10^{6} \mathrm{~Hz}, \mathbf{L}_{\text {loop }}$ decreases with frequency while $\mathbf{C}_{\text {loop }}$ slightly increases with frequency. The maximum rate of change for $\mathbf{L}_{\text {loop }}$ is about $50 \%$ for diagonals and $85 \%$ for off-diagonals, whereas for $\mathbf{C}_{\text {loop }}$ is about $8 \%$ for diagonals and $50 \%$ for off-diagonals. With a reasonable compromise that in $\mathbf{C}_{\text {loop }}$ the diagonals can be considered constant (to comply with the TEM assumption) and the off-diagonals are less dominant with respect to the diagonals when compared to the off-diagonals of $\mathbf{L}_{\text {loop }}$, we use the 

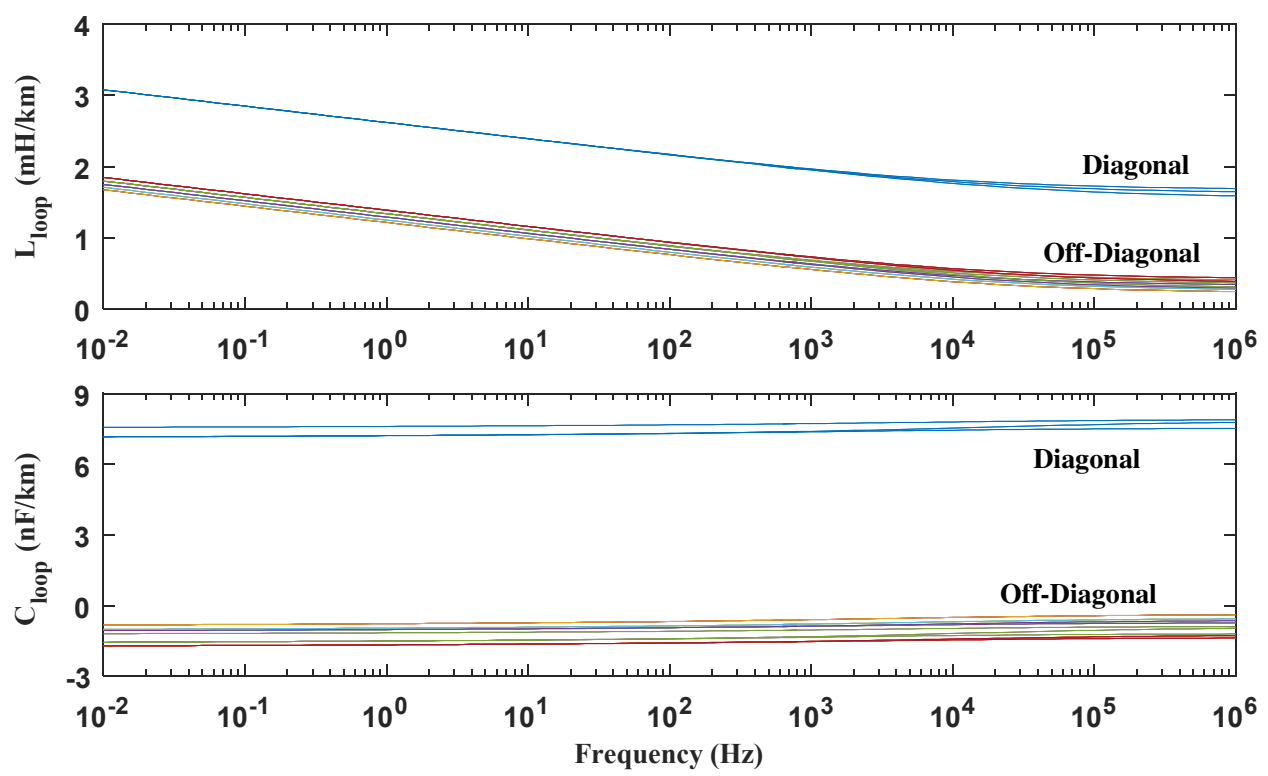

Figure 4.13: $\mathbf{L}_{\text {loop }}$ and $\mathbf{C}_{\text {loop }}$ for the line of Fig. 3.5.

skin effect in the capacitances to further investigate the RMTL equations.

First, we evaluate the effect of considering the skin effect in the capacitances on the wave collocation condition (4.12). Figure 4.14 compares $\mathbf{Z Y}(\mathbf{Y Z})^{-1}$ for the line of Fig. 3.5 for two conditions: 1) no skin effect in the capacitances $\left.\left(\mathbf{C}_{\text {ext }}\right), 2\right)$ with skin effect in the capacitances $\left(\mathbf{C}_{\text {loop }}\right)$. The curves in Fig. 4.14 are obtained using the RMTL equations for both conditions.

These results signify that considering the "skin effect" has a significant impact on the wave collocation condition by reducing the error of $\mathbf{Z Y}(\mathbf{Y Z})^{-1}$ to the maximum of about $0.1 \%$ for the real part (barely visible in the plot) and $0.5 \%$ for the imaginary part.

Next, we compare the effect of considering the skin effect in the capacitances on the product of the inductance and capacitance matrices. This product is normalized by $v_{c}^{2}$, where $v_{c}$ is the speed of light $\left(3 \times 10^{8} \mathrm{~m} / \mathrm{s}\right)$. The diagonal and off-diagonal elements of the product of inductance and capacitance matrices are plotted in Fig. 4.15.

As observed in Fig. 4.15, the diagonal terms of the normalized $\mathbf{L}_{\text {loop }} \mathbf{C}_{\text {loop }}$ are "One" and the off-diagonal terms are "Zero" for all frequencies. These results 

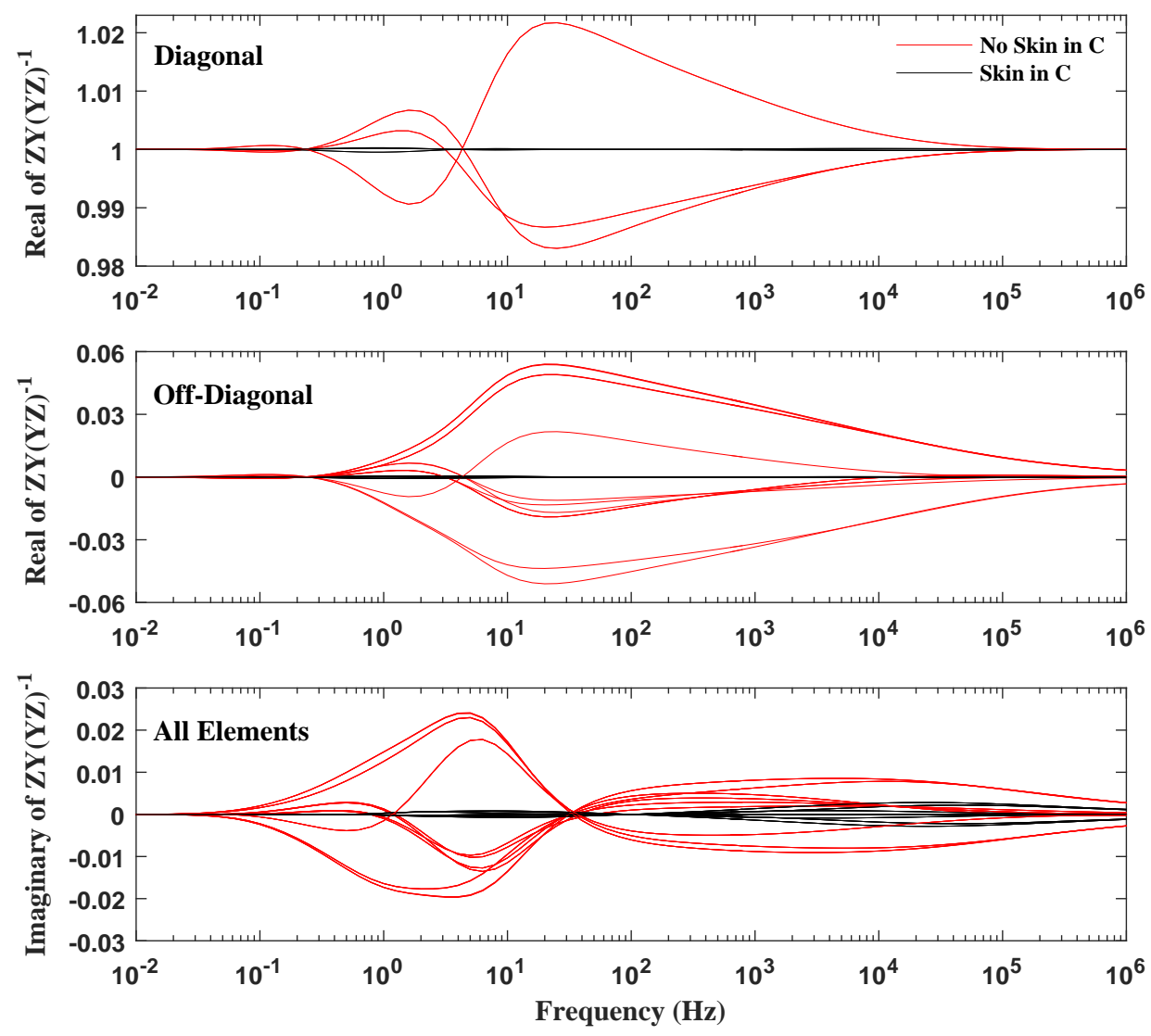

Figure 4.14: Validity of the collocation condition. If $\mathbf{Z Y}=\mathbf{Y Z}$, the product $\mathbf{Z Y}(\mathbf{Y Z})^{-1}$ should be one for the diagonals and zero for the offdiagonals.

seem to comply with physics that the propagation function only depends on the medium $\left(L C=\mu_{0} \varepsilon_{0}=1 / v^{2}\right)$. On the other hand, the elements of the normalized $\mathbf{L}_{\text {loop }} \mathbf{C}_{\text {ext }}$ vary with frequency until very high frequencies that both inductance and capacitance matrices are at their electrostatic position values.

With this premise, we use the skin effect in the capacitances to further explore the time domain transient simulation. For this analysis, we run the simulation condition of of Fig. 4.8a for the case that skin effect is considered in capacitances. Figure 4.16 is used to test two conceptual in these experiments:

i) MTL models (using two complex frequency dependent $\mathbf{T}_{V}(\omega)$ and $\mathbf{T}_{I}(\omega)$ ) 

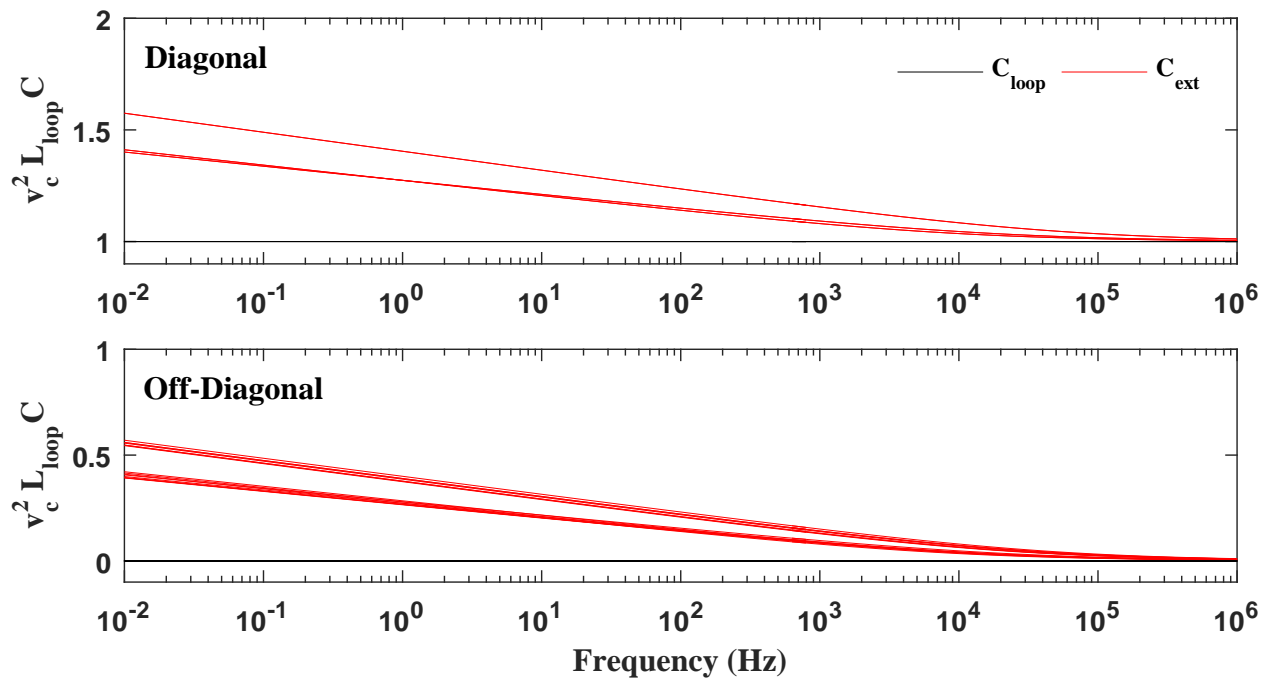

Figure 4.15: The effect of skin effect in capacitances on the product $\mathbf{L C}$ for the double circuit vertical line of Fig. 3.5.

versus RMTL models (using a constant real T).

ii) No skin effect in the capacitances (Fig. 4.16a) versus skin effect in the capacitances (Fig 4.16b).

Results of Fig. 4.16b suggest that including skin effect in the capacitances will further enforce the collocation wave condition as the MTL and RMTL models coincide. As opposed to Fig. 4.16a, there is no shift between the MTL and RMTL models when skin effect is considered in capacitances. In other words, collocation of charges and currents naturally contribute in the collocation of voltage and current waves (with no enforcement).

Figure 4.17 uses DTFS to compare the difference between the MTL equations with no skin effect in the $\mathbf{C}$ versus RMTL equations with skin effect in $\mathbf{C}$. 

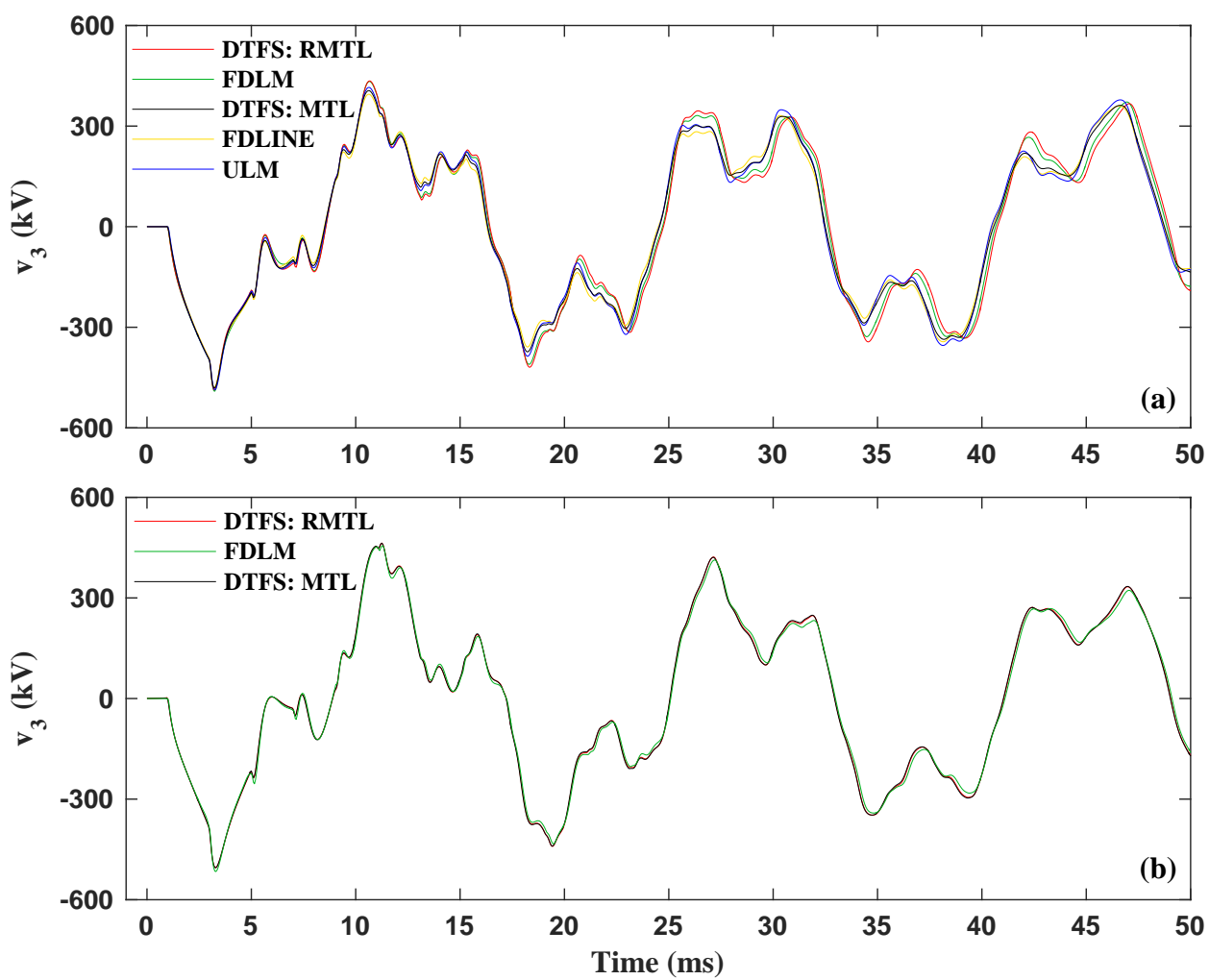

Figure 4.16: Comparison of MTL and RMTL models for two conditions: a) no skin in $\mathbf{C}$, b) with skin in C. $v_{3}$ in the test of Fig. 3.6.

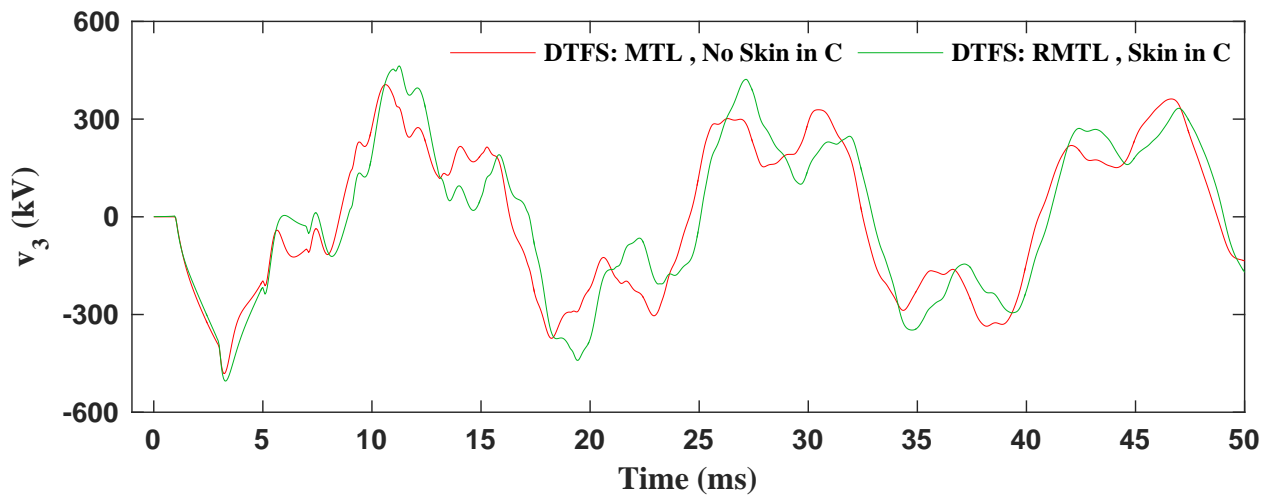

Figure 4.17: Comparison of MTL with $\mathbf{C}_{\text {ext }}$ versus RMTL with $\mathbf{C}_{\text {loop }} . v_{3}$ in the test of Fig. 3.6. 
Even though the results presented in this section are very promising, the validity of this premise still remains under the speculation since the equations that we used for this investigation are based on the TEM assumption that do not allow frequency dependent capacitances.

As observed in Fig. 4.17, considering skin effect in the capacitances has a stronger influence in the simulation results. Further research is required to discover the equations that allow us to consider skin effect in the capacitances. Ultimate validation can be performed by comparing the results with a field test. 


\section{Chapter 5}

\section{Summary of Contributions and Future Works}

\subsection{Conclusions and Contributions}

The main objective in this research was to develop a frequency dependent EMTP transmission line model which, in addition to preserving high accuracy, numerical stability, high computational efficiency, and simplicity in implementation, coincides well with the fundamentals of electromagnetics. The second objective was to verify the accuracy of the new line model with an accurate frequency domain reference solution. The steps taken to reach these objectives are as follows:

\section{Contribution 1}

The first step was to assess the accuracy of FDLINE model under asymmetrical line configurations. In Chapter 2, six different line configurations were simulated under asymmetrical short-circuit conditions using FDLINE and ULM. Three cases of three-phase lines were considered: single-circuit lines, double-circuit lines in the same tower, and double-circuit lines in separate towers. Open-circuit voltages and short-circuit currents were compared. The accuracy of time domain simulations were assessed using a conventional frequency-domain NLT solution. For all cases, the FDLINE model gave similar results to the ULM model and both models gave good results when compared to the reference conventional NLT solution 
for single-circuit lines. These results indicate that, contrary to traditional belief, a constant transformation matrix model like FDLINE is capable of representing multicircuit asymmetrical line configurations. With this validation, FDLINE was used in Chapter 4 to develop a new line model based on the RMTL equations.

In the simulation of double circuit lines in Chapter 2, we observed that the results obtained with NLT solution did not match with the EMTP line models. This happened because the choices of damping factor and windowing function for NLT were not suitable for those simulations.

\section{Contribution 2}

In Chapter 3, a simpler methodology based on the DTFS formulation was presented which provides very accurate solutions without the need, unlike the NLT, to adjust additional parameters. The main premise for the application of DTFS is that the user specifies a time window width and a frequency window width as starting points for the solution. The time window width is determined by the system time constants and the frequency window width is determined by the desired maximum frequency of the transient. Guidelines were provided for this set up. This approach guarantees a one-to-one matching between time points and frequency points and avoids the typical numerical errors of frequency domain solutions such as aliasing and Gibbs. The accuracy of the DTFS was tested for a variety of simulations including circuits with lumped elements and frequency dependent transmission lines.

\section{Contribution 3}

In Chapter 4, a new EMTP transmission line "FDLM" was developed that physically satisfies the symmetry for the propagation of voltage waves and current waves referred to as "collocation wave condition". This condition was reached by using a single transformation matrix as a constraint to diagonalize the wave functions. The validity of this approximation was tested mathematically with high accuracy. The nature of real and constant of the transformation matrix within wide range of switching transients frequency further facilitated the diagonalization. Based on these considerations, the RMTL equations were proposed which were further used in the development of the FDLM model. Unifying the slightly different elements of the ground return resistance matrix given by the method of image or Carson's for- 
mula resulted in physical consistency and higher symmetry in the RMTL equations.

Simulation results validated the accuracy of the FDLM compared to FDLINE, ULM, and newly developed DTFS reference solution for the double circuit vertical line. In terms of computational cost, FDLM is as efficient as FDLINE while using a constant real transformation matrix is not an approximation but is exact over the entire frequency range for switching transients.

\subsection{Future Works}

The accuracy and functionality of the proposed FDLM model can be more justified with performing the following studies:

\section{Study 1: Inclusion of ground wires and bundled conductors in the model}

Ground wires are steel conductors used to protect the overhead transmission lines from the lightning. These conductors can be grounded at both ends of towers and they are eliminated from the matrix of impedance and admittance by using as for example a Kron reduction when calculating line parameters. Since the radius and DC resistance of ground wires are different from the conductors, the effect of matrix reduction may cause some asymmetries in the model. This is also the case for bundled conductors when matrix reduction is used to propagate the effect of bundling on other phases.

When ground wires are segmented, they may contribute to the admittance matrix creating another source of asymmetry between the impedance and admittance matrices. These aspects should be investigated in the future work.

\section{Study 2: Test the RMTL model for underground cables}

As addressed in [71], in the MTL model the transformation matrices will become strongly frequency dependent in the case of the underground cables due to the short distances from the conductors to the ground. As further extension to this research, it would be interesting to reformulate the cable equations based on the proposed RMTL model and investigate the behaviour of the transformation matrices and the validity of the collocation wave condition. 


\section{Study 3: Test the RMTL model for a field test}

In this thesis, the proposed FDLM passed the preliminary verifications through comparisons with the DTFS reference frequency domain solution for the same system topology and parameters. Since this model belongs to the new class of line equations where voltage and current waves are collocated, the final verification will be achieved by comparing the transient solution of the new line model with a field test with an untransposed asymmetrical line configuration. This requires to access to accurate measurement of the field test voltages and currents, line configuration, conductor type, equivalent source representation, and earth resistance.

\section{Study 4: Collocation of charges and currents}

One consideration to incorporate additional symmetry into the line equations is the principle of collocation of charge and current, which would require the recollocation of the charge in the conductors and the ground when the current position is affected by the skin effect. In this research, we investigated this concept within certain validity of TEM assumption. More fundamental research is required to find the line equations that can allow the use of skin effect in capacitances. 


\section{Bibliography}

[1] Improvements on the JMARTI transmission Line Model. DCG-EPRI Coordination Group, 1983. $\rightarrow$ pages 2, 9, 23, 93, 97

[2] Microtran Power Systems Analysis Corporation, MicroTran-Transient Analysis Program Reference Manual, Vancouver, 2014. $\rightarrow$ pages 32, 72

[3] H. Abdollahzadeh, M. Jazaeri, and A. Tavighi. A new fast-converged estimation approach for dynamic voltage restorer (dvr) to compensate voltage sags in waveform distortion conditions. International Journal of Electrical Power \& Energy Systems, 54:598-609, 2014. $\rightarrow$ pages 49

[4] F. L. Alvarado and R. Betancourt. An accurate closed-form approximation for ground return impedance calculations. Proceedings of the IEEE, 71(2): 279-280, February 1983. $\rightarrow$ pages 4

[5] A. Ametani. The application of the fast fourier transform to electrical transient phenomena. International Journal of Electrical Engineering Education, 10(4):277-287, 1973. $\rightarrow$ pages 7

[6] A. Ametani. Stratified earth effects on wave propagation frequency dependent parameters. IEEE Transactions on Power Apparatus and Systems, PAS-93(5):1233-1239, September 1974. $\rightarrow$ pages 4

[7] A. Ametani and K. Imanishi. Development of exponential fourier transform and its application to electrical transients. Proceedings of the Institution of Electrical Engineers, 126(1):51-56, January 1979. $\rightarrow$ pages 7

[8] A. Ametani and R. Schinzinger. Equations for surge impedance and propagation constant of transmission lines above stratified earth. IEEE Transactions on Power Apparatus and Systems, 95(3):773-781, May 1976. $\rightarrow$ pages 4 
[9] A. Arismunandar. Capacitive correction factors for transmission lines to include finite conductivity and dielectric constant of the earth. IEEE Transsactions on Power Apparatus and Systems, pages 436-456, 1963. $\rightarrow$ pages 5

[10] L. J. B. Bergeron. Water hammer in hydraulics and wave surges in electricity. Wiley, 1961. $\rightarrow$ pages 8

[11] J.-F. Blais, M. Cimmino, A. Ross, and D. Granger. Suppression of time aliasing in the solution of the equations of motion of an impacted beam with partial constrained layer damping. Journal of Sound and Vibration, 326(3-5):870-882, October 2009. $\rightarrow$ pages 55

[12] A. Budner. Introduction of frequency-dependent line parameters into an electromagnetic transients program. IEEE Transactions on Power Apparatus and Systems, PAS-89(1):88-97, January 1970. $\rightarrow$ pages 8

[13] J. R. Carson. Wave propagation in overhead wires with ground return. Bell System Technical Journal, 5(4):539-554, October 1926. $\rightarrow$ pages 3, 82

[14] F. Castellanos and J. R. Martí. Phase-domain multi-phase transmission line models. International Conference on Power System Transients (IPST 95), Lisbon, Portugal, pages 17-22, 3-7 September 1995. $\rightarrow$ pages 11

[15] F. Castellanos and J. R. Martí. Full frequency-dependent phase-domain transmission line model. IEEE Transactions on Power Systems, 12(3): 1331-1339, August 1997. $\rightarrow$ pages 10

[16] A. Chinea and S. Grivet-Talocia. A passivity enforcement scheme for delay-based transmission line macromodels. IEEE Microwave and Wireless Components Letters, 17(8):562-564, August 2007. $\rightarrow$ pages 11

[17] A. I. Chrysochos, T. A. Papadopoulos, and G. K. Papagiannis. Enhancing the frequency-domain calculation of transients in multiconductor power transmission lines. Electric Power Systems Research, 122:56-64, 2015. $\rightarrow$ pages 7

[18] S. Cristina and M. D'Amore. Dft-based procedure for transmission-line transient computation. IEE Proceedings C-Generation, Transmission and Distribution, 134(2):138-144, March 1987. $\rightarrow$ pages 7

[19] C. G. Cullen. Matrices and linear transformations. Courier Corporation, 2012. $\rightarrow$ pages 10 
[20] M. D'Amore and M. S. Sarto. A new formulation of lossy ground return parameters for transient analysis of multiconductor dissipative lines. IEEE Transactions on Power Delivery, 12(1):303-314, January 1997. $\rightarrow$ pages 6

[21] M. D'Amore and M. S. Sarto. Simulation models of a dissipative transmission line above a lossy ground for a wide-frequency range. i. single conductor configuration. IEEE Transactions on Electromagnetic Compatibility, 38(2):127-138, May 1996. $\rightarrow$ pages 6

[22] M. D'Amore and M. S. Sarto. Simulation models of a dissipative transmission line above a lossy ground for a wide-frequency range. ii. multiconductor configuration. IEEE Transactions on Electromagnetic Compatibility, 38(2):139-149, May 1996. $\rightarrow$ pages 6

[23] S. J. Day, N. Mullineux, and J. R. Reed. Developments in obtaining transient response using fourier transforms part i: Gibbs phenomena and fourier integrals. International Journal of Electrical Engineering Education, 3(4):501-506, 1965. $\rightarrow$ pages 7

[24] S. J. Day, N. Mullineux, and J. R. Reed. Developments in obtaining transient response using fourier transforms part ii: Use of the modified fourier transform. International Journal of Electrical Engineering Education, 4(1):31-40, 1966. $\rightarrow$ pages 7

[25] H. M. J. De Silva, A. M. Gole, J. E. Nordstrom, and L. M. Wedepohl. Robust passivity enforcement scheme for time-domain simulation of multi-conductor transmission lines and cables. IEEE Transactions on Power Delivery, 25(2):930-938, April 2010. $\rightarrow$ pages 11

[26] A. Deri, G. Tevan, A. Semlyen, and A. Castanheira. The complex ground return plane a simplified model for homogeneous and multi-layer earth return. IEEE Transactions on Power Apparatus and Systems, PAS-100(8): 3686-3693, August 1981. $\rightarrow$ pages 3, 5, 16

[27] D. Deschrijver, B. Gustavsen, and T. Dhaene. Causality preserving passivity enforcement for traveling-wave-type transmission-line models. IEEE Transactions on Power Delivery, 24(4):2461-2462, October 2009. $\rightarrow$ pages 11

[28] H. W. Dommel. Electromagnetic transients program (EMTP) theory book. Bonneville Power Administration, 1986. $\rightarrow$ pages 2, 41 
[29] H. W. Dommel. Digital computer solution of electromagnetic transients in single and multiphase networks. IEEE Transactions on Power Apparatus and Systems, PAS-88(4):388-399, April 1969. $\rightarrow$ pages 2

[30] H. W. Dommel and W. S. Meyer. Computation of electromagnetic transients. Proceedings of the IEEE, 62(7):983-993, July 1974. $\rightarrow$ pages 2

[31] C. Dubanton. Calcul approché des parametres primaires et secondaires dune ligne de transport. EDF Bulletin de la Direction des Etudes et Recherches, 1:53-62, 1969. $\rightarrow$ pages 3

[32] C. Dufour, H. Le-Huy, J. C. Soumagne, and A. El Hakimi. Real-time simulation of power transmission lines using martí model with optimal fitting on dual-dsp card. IEEE Transactions on Power Delivery, 11(1): 412-419, January 1996. $\rightarrow$ pages 9

[33] A. E. Efthymiadis and L. M. Wedepohl. Propagation characteristics of infinitely-long single-conductor lines by the complete field solution method. Proceedings of the Institution of Electrical Engineers, 125(6): 511-517, June 1978. $\rightarrow$ pages 6

[34] E. P. R. I. (EPRI). Transmission-line reference book. $345 \mathrm{kv}$ and above. Technical report, 2nd Edition, General Electric Co., Pittsfield, MA (USA). Large Transformer Div.; General Electric Co., Schenectady, NY (USA). Electric Utility Systems Engineering Dept., 1982. $\rightarrow$ pages 28, 51, 94

[35] J. C. Escamilla, P. Moreno, and P. Gómez. New model for overhead lossy multiconductor transmission lines. IET Generation, Transmission \& Distribution, 7(11):1185-1193, 2013. $\rightarrow$ pages 10

[36] J. A. B. Faria and J. F. B. Da Silva. Wave propagation in polyphase transmission lines a general solution to include cases where ordinary modal theory fails. IEEE Transactions on Power Delivery, 1(2):182-189, April 1986. $\rightarrow$ pages 9

[37] J. A. B. Faria and J. F. B. da Silva. The effect of randomly earthed ground wires on plc transmission-a simulation experiment. IEEE Transactions on Power Delivery, 5(4):1669-1677, November 1990. $\rightarrow$ pages 10

[38] J. B. Faria. Overhead three-phase transmission lines-nondiagonalizable situations. IEEE Transactions on Power Delivery, 3(4):1348-1355, October 1988. $\rightarrow$ pages 9 
[39] A. B. Fernandes, W. L. Neves, E. G. Costa, and M. N. Cavalcanti. Transmission line shunt conductance from measurements. IEEE Transactions on Power Delivery, 19(2):722-728, 2004. $\rightarrow$ pages 16, 51

[40] R. H. Galloway, W. B. Shorrocks, and L. M. Wedepohl. Calculation of electrical parameters for short and long polyphase transmission lines. Proceedings of the Institution of Electrical Engineers, 111(12):2051-2059, 1964. $\rightarrow$ pages 5

[41] C. Gary. Approche complète de la propagation multifilaire en haute fréquence par utilisation des matrices complexes. EDF Bulletin de la Direction des Etudes et Recherches, 3(4):5-20, 1976. $\rightarrow$ pages 3

[42] P. Gómez and J. C. Escamilla. Frequency domain modeling of nonuniform multiconductor lines excited by indirect lightning. International Journal of Electrical Power \& Energy Systems, 45(1):420-426, 2013. $\rightarrow$ pages 7

[43] P. Gómez and F. A. Uribe. The numerical laplace transform: An accurate technique for analyzing electromagnetic transients on power system devices. International Journal of Electrical Power \& Energy Systems, 31 (2-3):116-123, February-March 2009. $\rightarrow$ pages 7, 26, 27

[44] P. Gómez, P. Moreno, and J. L. Naredo. Frequency-domain transient analysis of nonuniform lines with incident field excitation. IEEE Transactions on Power Delivery, 20(3):2273-2280, July 2005. $\rightarrow$ pages 7

[45] B. Gustavsen. Validation of frequency-dependent transmission line models. IEEE Transactions on Power Delivery, 20(2):925-933, April 2005. $\rightarrow$ pages 7

[46] B. Gustavsen. Improving the pole relocating properties of vector fitting. IEEE Transactions on Power Delivery, 21(3):1587-1592, July 2006. $\rightarrow$ pages 11,25

[47] B. Gustavsen. Avoiding numerical instabilities in the universal line model by a two-segment interpolation scheme. IEEE Transactions on Power Delivery, 28(3):1643-1651, July 2013. $\rightarrow$ pages 11

[48] B. Gustavsen. Passivity enforcement for transmission line models based on the method of characteristics. IEEE Transactions on Power Delivery, 23 (4):2286-2293, October 2008. $\rightarrow$ pages 11 
[49] B. Gustavsen. Modal domain-based modeling of parallel transmission lines with emphasis on accurate representation of mutual coupling effects. IEEE Transactions on Power Delivery, 27(4):2159-2167, October 2012. $\rightarrow$ pages 10,28

[50] B. Gustavsen and J. Nordstrom. Pole identification for the universal line model based on trace fitting. IEEE Transactions on Power Delivery, 23(1): 472-479, January 2008. $\rightarrow$ pages 11

[51] B. Gustavsen and A. Semlyen. Combined phase and modal domain calculation of transmission line transients based on vector fitting. IEEE Transactions on Power Delivery, 13(2):596-604, April 1998. $\rightarrow$ pages 11

[52] B. Gustavsen and A. Semlyen. Simulation of transmission line transients using vector fitting and modal decomposition. IEEE Transactions on Power Delivery, 13(2):605-614, April 1998. $\rightarrow$ pages 11

[53] B. Gustavsen and A. Semlyen. Enforcing passivity for admittance matrices approximated by rational functions. IEEE Transactions on Power Systems, 16(1):97-104, February 2001. $\rightarrow$ pages 11

[54] B. Gustavsen and A. Semlyen. Calculation of transmission line transients using polar decomposition. IEEE Transactions on Power Delivery, 13(3): 855-862, July 1998. $\rightarrow$ pages 11

[55] B. Gustavsen and A. Semlyen. Rational approximation of frequency domain responses by vector fitting. IEEE Transactions on Power Delivery, 14(3):1052-1061, July 1999. $\rightarrow$ pages 2, 11, 23

[56] D. E. Hedman. Propagation on overhead transmission lines: I-theory of modal analysis, ii-earth conduction effects and practical results. IEEE Transactions on Power Apparatus and Systems, 84(3):200-211, March 1965. $\rightarrow$ pages 5, 8, 20,86

[57] W. Hendrickx and T. Dhaene. A discussion of "rational approximation of frequency domain responses by vector fitting". IEEE Transactions on Power Systems, 21(1):441-443, February 2006. $\rightarrow$ pages 24

[58] L. Hofmann. Series expansions for line series impedances considering different specific resistances, magnetic permeabilities, and dielectric permittivities of conductors, air, and ground. IEEE Transactions on Power Delivery, 18(2):564-570, April 2003. $\rightarrow$ pages 3 
[59] K. Iwamoto. Use of travelling waves on the measurement of earth resistivity. Journal of Institution of Electrical Engineers of Japan, 78: 1038-1049, 1958. $\rightarrow$ pages 4

[60] K. Iwamoto. Wide time-domain calculation of energizing surges in multi-conductor transmission line by a method of logarithmic fourier transformation. IEEE Transactions on Power Apparatus and Systems, PAS-100(4):1788-1795, April 1981. $\rightarrow$ pages 7

[61] H. Keshtkar, S. K. Solanki, and J. M. Solanki. Improving the accuracy of impedance calculation for distribution power system. IEEE Transactions on Power Delivery, 29(2):570-579, April 2014. $\rightarrow$ pages 4

[62] H. Kikuchi. Wave propagation along an infinite wire above ground at high frequencies. Electrotechnical Journal of Japan, 2:73-78, 1956. $\rightarrow$ pages 5

[63] I. Kocar, J. Mahseredjian, and G. Olivier. Improvement of numerical stability for the computation of transients in lines and cables. IEEE Transactions on Power Delivery, 25(2):1104-1111, April 2010. $\rightarrow$ pages 11

[64] O. R. Leaños, J. L. Naredo, and P. Moreno. Assessment of approximate formulas for calculating overhead-line earth-impedances. 40th North American Power Symposium, pages 1-6, 28-30 September 2008. $\rightarrow$ pages 4

[65] F. J. Marcano. Modelling of transmission lines using idempotent decomposition. PhD thesis, University of British Columbia, August 1996.

$\rightarrow$ pages 11

[66] F. J. Marcano and J. R. Martí. Idempotent line model: Case studies. International Conference on Power System Transients (IPST 97), Seattle, Washington, pages 67-72, 22-26 June 1997. $\rightarrow$ pages 11, 28

[67] J. R. Martí. Digital signal processing from a simulation point of view. class notes for elec 466, 1999. $\rightarrow$ pages 41

[68] J. R. Martí. The problem of frequency dependence in transmission line modelling. $\mathrm{PhD}$ thesis, University of British Columbia, April 1981. $\rightarrow$ pages $2,8,9,23$

[69] J. R. Martí. Accurate modelling of frequency-dependent transmission lines in electromagnetic transient simulations. IEEE Transactions on Power 
Apparatus and Systems, PAS-101(1):147-157, January 1982. $\rightarrow$ pages 2 , 9, 22, 23, 92, 99

[70] J. R. Martí, H. W. Dommel, L. Martí, and V. Brandwajn. Approximate transformation matrices for unbalanced transmission lines. 9th Power System Computation Conference (PSCC 87), Butterworths, London, pages 416-422, August 1987. $\rightarrow$ pages 9

[71] L. Martí. Simulation of transients in underground cables with frequency-dependent modal transformation matrices. IEEE Transactions on Power Delivery, 3(3):1099-1110, July 1988. $\rightarrow$ pages 2, 10, 109

[72] L. Martí, R. Y. Brierley, and T. E. Grainger. Analysis of electromagnetic transients in cross-bounded cable systems using frequency dependent cable models. International Conference on Power System Transients (IPST 95), Lisbon, Portugal, pages 5-10, 3-7 September 1995. $\rightarrow$ pages 10

[73] W. S. Meyer and H. W. Dommel. Numerical modelling of frequency-dependent transmission-line parameters in an electromagnetic transients program. IEEE Transactions on Power Apparatus and Systems, PAS-93(5):1401-1409, September 1974. $\rightarrow$ pages 8

[74] A. Morched, B. Gustavsen, and M. Tartibi. A universal model for accurate calculation of electromagnetic transients on overhead lines and underground cables. IEEE Transactions on Power Delivery, 14(3): 1032-1038, July 1999. $\rightarrow$ pages 2, 11, 24

[75] P. Moreno and A. Ramirez. Implementation of the numerical laplace transform: a review task force on frequency domain methods for emt studies, working group on modeling and analysis of system transients using digital simulation, general systems subcommittee, ieee power engineering society. IEEE Transactions on Power Delivery, 23(4):2599-2609, October 2008. $\rightarrow$ pages 7, 25, 26, 55

[76] P. Moreno, P. Gómez, J. L. Naredo, and J. L. Guardado. Frequency domain transient analysis of electrical networks including non-linear conditions. International Journal of Electrical Power \& Energy Systems, 27(2): 139-146, February 2005. $\rightarrow$ pages 7

[77] P. Moreno, R. de la Rosa, and J. L. Naredo. Frequency domain computation of transmission line closing transients. IEEE Transactions on Power Delivery, 6(1):275-281, January 1991. $\rightarrow$ pages 7 
[78] N. Mullineux, J. R. Reed, and L. M. Wedepohl. Calculation of electrical parameters for short and long polyphase transmission lines. Proceedings of the Institution of Electrical Engineers, 112(4):741-742, April 1965. $\rightarrow$ pages 4

[79] N. Nagaoka and A. Ametani. A development of a generalized frequency-domain transient program-ftp. IEEE Transactions on Power Delivery, 3(4):1996-2004, October 1988. $\rightarrow$ pages 7

[80] M. Nakagawa. Further studies on wave propagation along overhead transmission lines: Effects of admittance correction. IEEE Transactions on Power Apparatus and Systems, PAS-100(7):3626-3633, July 1981. $\rightarrow$ pages 5,6

[81] M. Nakagawa. Admittance correction effects of a single overhead line. IEEE Transactions on Power Apparatus and Systems, PAS-100(3): 1154-1161, March 1981. $\rightarrow$ pages 5, 6, 100

[82] M. Nakagawa and K. Iwamoto. Earth-return impedance for the multi-layer case. IEEE Transactions on Power Apparatus and Systems, 95(2):671-676, April 1976. $\rightarrow$ pages 4

[83] M. Nakagawa, A. Ametani, and K. Iwamoto. Further studies on wave propagation in overhead lines with earth return: impedance of stratified earth. Proceedings of the Institution of Electrical Engineers, 120(12): 1521-1528, December 1973. $\rightarrow$ pages 4

[84] J. L. Naredo, A. C. Soudack, and J. R. Martí. Simulation of transients on transmission lines with corona via the method of characteristics. IEE Proceedings-Generation, Transmission and Distribution, 142(1):81-87, January 1995. $\rightarrow$ pages 10

[85] H. V. Nguyen, H. W. Dommel, and J. R. Martí. Direct phase-domain modelling of frequency-dependent overhead transmission lines. IEEE Transactions on Power Delivery, 12(3):1335-1342, July 1997. $\rightarrow$ pages 10

[86] T. Noda. Numerical techniques for accurate evaluation of overhead line and underground cable constants. IEEJ Transactions on Electrical and Electronic Engineering, 3(5):549-559, August 2008. $\rightarrow$ pages 4

[87] T. Noda. A double logarithmic approximation of carson's ground-return impedance. IEEE Transactions on Power Delivery, 21(1):472-479, January 2005. $\rightarrow$ pages 4 
[88] T. Noda, N. Nagaoka, and A. Ametani. Phase domain modeling of frequency-dependent transmission lines by means of an arma model. IEEE Transactions on Power Delivery, 11(1):401-411, January 1996. $\rightarrow$ pages 10,25

[89] R. Nuricumbo-Guillen, P. Gomez, F. P. Espino-Cortes, and F. A. Uribe. Accurate computation of transient profiles along multiconductor transmission systems by means of the numerical laplace transform. IEEE Transactions on Power Delivery, 29(5):2385-2393, 2014. $\rightarrow$ pages 7

[90] T. A. Papadopoulos, G. K. Papagiannis, and D. A. Labridis. Wave propagation characteristics of overhead conductors above imperfect stratified earth for a wide frequency range. IEEE Transactions on Magnetics, 45(3):1064-1067, March 2009. $\rightarrow$ pages 6

[91] G. K. Papagiannis, D. A. Tsiamitros, D. P. Labridis, and P. S. Dokopoulos. A systematic approach to the evaluation of the influence of multilayered earth on overhead power transmission lines. IEEE Transactions on Power Delivery, 20(4):2594-2601, October 2005. $\rightarrow$ pages 4

[92] A. Papoulis. The Fourier Integral and its Applications. McGraw-Hill, New York, 1962. $\rightarrow$ pages 23

[93] C. R. Paul. Analysis of multiconductor transmission lines. 2nd Edition, John Wiley \& Sons, 2008. $\rightarrow$ pages 19, 79, 80

[94] P. Pettersson. Image representation of wave propagation on wires above, on and under ground. IEEE Transactions on Power Delivery, 9(2):1049-1055, April 1994. $\rightarrow$ pages 6

[95] P. Pettersson. Propagation of waves on a wire above a lossy ground-different formulations with approximations. IEEE Transactions on Power Delivery, 14(3):1173-1180, July 1999. $\rightarrow$ pages 6

[96] M. Pizarro and R. Eriksson. Modeling of the ground mode of transmission lines in time domain simulations. 7th international symposium on high voltage engineering, (ISH 91), pages 179-182, 26-30 August 1991. $\rightarrow$ pages 4

[97] F. Pollaczek. On the induction effects of a single phase ac line. Electrische Nachricten Technik, 4:18-30, April 1927. $\rightarrow$ pages 3 
[98] F. Pollaczek. On the field produced by an infinitely long wire carrying alternating current. Electrische Nachricten Technik, 3:339-359, March 1926. $\rightarrow$ pages 3

[99] J. G. Proakis and D. G. Manolakis. Digital Signal Processing: Principles, Algotithms, and Applications. 3rd Edition, Prentice Hall, 1996. $\rightarrow$ pages 49

[100] A. Ramirez and F. Uribe. A broad range algorithm for the evaluation of carson's integral. IEEE Transactions on Power Delivery, 22(2):1188-1193, April 2007. $\rightarrow$ pages 4

[101] A. Ramirez, P. Gomez, P. Moreno, and A. Gutierrez. Frequency domain analysis of electromagnetic transients through the numerical laplace transforms. In Power Engineering Society General Meeting (PES 2004), pages 1136-1139. IEEE, 2004. $\rightarrow$ pages 7

[102] A. Ramírez, J. L. Naredo, and P. Moreno. Full frequency-dependent line model for electromagnetic transient simulation including lumped and distributed sources. IEEE Transactions on Power Delivery, 20(1):292-299, January 2005 . $\rightarrow$ pages 10

[103] C. Sanathanan and J. Koerner. Transfer function synthesis as a ratio of two complex polynomials. IEEE Transactions on Automatic Control, 8(1): 56-58, January 1963. $\rightarrow$ pages 24

[104] A. Semlyen. Some frequency domain aspects of wave propagation on nonuniform lines. IEEE Transactions on Power Delivery, 18(1):315-322, 2003. $\rightarrow$ pages 7

[105] A. Semlyen. Correspondence: Approximation to carson's loss formulae. Canadian Electrical Engineering Journal, 6(2):30-31, April 1981. $\rightarrow$ pages 3

[106] A. Semlyen. Ground return parameters of transmission lines an asymptotic analysis for very high frequencies. IEEE Transactions on Power Apparatus and Systems, PAS-100(3):1031-1038, March 1981. $\rightarrow$ pages 3

[107] A. Semlyen and A. Dabuleanu. Fast and accurate switching transient calculations on transmission lines with ground return using recursive convolutions. IEEE Transactions on Power Apparatus and Systems, 94(2): 561-571, March 1975. $\rightarrow$ pages 9 
[108] A. Semlyen and B. Gustavsen. Phase-domain transmission-line modeling with enforcement of symmetry via the propagated characteristic admittance matrix. IEEE Transactions on Power Delivery, 27(2):626-631, April 2012. $\rightarrow$ pages 11,80

[109] A. Semlyen and A. Ramirez. Direct frequency domain computation of transmission line transients due to switching operations. IEEE Transactions on Power Delivery, 23(4):2255-2261, October 2008. $\rightarrow$ pages 7

[110] A. Semlyen and D. Shirmohammadi. Calculation of induction and magnetic field effects of three phase overhead lines above homogeneous earth. IEEE Transactions on Power Apparatus and Systems, PAS-101(8): 2747-2754, September 1982. $\rightarrow$ pages 3

[111] J. K. Snelson. Propagation of travelling waves on transmission lines-frequency dependent parameters. IEEE Transactions on Power Apparatus and Systems, PAS-91(1):85-91, January 1972. $\rightarrow$ pages 8

[112] A. Sommerfeld. Propagation of waves in wireless telegraphy. Ann. der Phys, 28(3):665-736, March 1909. $\rightarrow$ pages 3

[113] E. D. Sunde. Earth conduction effects in transmission systems. Dover Publications Inc., 1949. $\rightarrow$ pages 4, 5

[114] M. C. Tavares, J. Pissolato, and C. M. Portela. Mode domain multiphase transmission line model-use in transient studies. IEEE Transactions on Power Delivery, 14(4):1533-1544, 1999. $\rightarrow$ pages 10

[115] A. Tavighi, S. Soleymani, J. Martí, and H. Abdollahzadeh. Swift detection of power transformer inrush current based on the windowed-adaptive linear combiner estimation algorithm. $\rightarrow$ pages 49

[116] A. Tavighi, J. R. Martí, and J. A. Gutierrez-Robles. Comparison of the fdline and ulm frequency dependent emtp line models with a reference laplace solution. International Conference on Power System Transients (IPST 15), Cavtat, Croatia, 15-18 June 2015. $\rightarrow$ pages 50, 51, 94, 96, 99

[117] A. Tavighi, H. Abdollahzadeh, and J. Martí. Fast response dvr control strategy design to compensate unbalanced voltage sags and swells in distribution systems. In Power and Energy Society General Meeting (PES), 2013 IEEE, pages 1-5. IEEE, 2013. $\rightarrow$ pages 49 
[118] T. Theodoulidis. On the closed-form expression of carson's integral. Periodica Polytechnica. Electrical Engineering and Computer Science, 59 (1):26-29, 2015. $\rightarrow$ pages 82

[119] D. A. Tsiamitros, G. K. Papagiannis, and P. S. Dokopoulos. Homogenous earth approximation of two-layer earth structures: An equivalent resistivity approach. IEEE Transactions on Power Delivery, 22(1):658-666, January 2007. $\rightarrow$ pages 4

[120] D. A. Tsiamitros, G. K. Papagiannis, and P. S. Dokopoulos. Earth return impedances of conductor arrangements in multilayer soils-part ii: numerical results. IEEE Transactions on Power Delivery, 23(4): 2401-2408, November 2008. $\rightarrow$ pages 4

[121] D. A. Tsiamitros, G. K. Papagiannis, and P. S. Dokopoulos. Earth return impedances of conductor arrangements in multilayer soils-part i: theoretical model. IEEE Transactions on Power Delivery, 23(4): 2392-2400, October 2008. $\rightarrow$ pages 4

[122] F. A. Uribe, J. L. Naredo, P. Moreno, and L. Guardado. Electromagnetic transients in underground transmission systems through the numerical laplace transform. International Journal of Electrical Power \& Energy Systems, 24(3):215-221, 2002. $\rightarrow$ pages 7

[123] S. Vujević and D. Lovrić. Inverse continuous numerical fourier transform for transient analysis of electromagnetic phenomena. IEEE Transactions on Electromagnetic Compatibility, 57(5):1149-1154, October 2015. $\rightarrow$ pages 41

[124] J. R. Wait. Electromagnetic wave theory. Harper \& Row, 1985. $\rightarrow$ pages 90

[125] J. R. Wait and K. P. Spies. On the image representation of the quasi-static fields of a line current source above the ground. Canadian Journal of Physics, 47(23):2731-2733, May 1969. $\rightarrow$ pages 6

[126] L. M. Wedepohl. Frequency domain analysis of wave propagation in multiconductor transmission systems. Lecture Notes, University of British Columbia, Department of Electrical and Computer Engineering, Canada, 1993. $\rightarrow$ pages 10, 19, 79

[127] L. M. Wedepohl. Power system transients: Errors incurred in the numerical inversion of the laplace transform. 26th Midwest Symposium on Circuits and Systems, (MSCS 83), pages 174-178, August 1983. $\rightarrow$ pages 7, 26 
[128] L. M. Wedepohl. Application of matrix methods to the solution of travelling-wave phenomena in polyphase systems. Proceedings of the Institution of Electrical Engineers, 110(12):2200-2212, December 1963. $\rightarrow$ pages $8,20,86$

[129] L. M. Wedepohl and A. E. Efthymiadis. Wave propagation in transmission lines over lossy ground: a new, complete field solution. Proceedings of the Institution of Electrical Engineers, 125(6):505-510, June 1978. $\rightarrow$ pages 6

[130] L. M. Wedepohl and C. S. Indulkar. Switching overvoltages in short crossbonded cable systems using the fourier transform. Proceedings of the Institution of Electrical Engineers, 122(11):1217-1221, November 1975. $\rightarrow$ pages 7

[131] L. M. Wedepohl and S. E. T. Mohamed. Transient analysis of multiconductor transmission lines with special reference to nonlinear problems. Proceedings of the Institution of Electrical Engineers, 117(5): 979-988, May 1970. $\rightarrow$ pages 7

[132] L. M. Wedepohl and S. E. T. Mohamed. Multiconductor transmission lines. theory of natural modes and fourier integral applied to transient analysis. Proceedings of the Institution of Electrical Engineers, 116(9):1553-1563, September 1969. $\rightarrow$ pages 7

[133] L. M. Wedepohl and R. G. Wasley. Wave propagation in multiconductor overhead lines. calculation of series impedance for multilayer earth. Proceedings of the Institution of Electrical Engineers, 113(4):627-632, April 1966. $\rightarrow$ pages 5

[134] L. M. Wedepohl and D. J. Wilcox. Transient analysis of underground power-transmission systems. system-model and wave-propagation characteristics. Proceedings of the Institution of Electrical Engineers, 120 (2):253-260, February 1973. $\rightarrow$ pages 7

[135] L. M. Wedepohl, H. V. Nguyen, and G. D. Irwin. Frequency-dependent transformation matrices for untransposed transmission lines using newton-raphson method. IEEE Transactions on Power Systems, 11(3): 1538-1546, August 1996. $\rightarrow$ pages 21, 68, 87

[136] D. J. Wilcox. Numerical laplace transformation and inversion. International Journal of Electrical Engineering Education, 15(3):247-265, July $1978 . \rightarrow$ pages 7,26 
[137] W. H. Wise. Propagation of high-frequency currents in ground return circuits. Proceedings of the Institute of Radio Engineers, 22(4):522-527, April 1934. $\rightarrow$ pages 5

[138] W. H. Wise. Potential coefficients for ground return circuits. Bell System Technical Journal, 27(2):365-371, April 1948. $\rightarrow$ pages 5, 100

[139] W. H. Wise. Effect of ground permeability on ground return circuits. Bell System Technical Journal, 10(3):472-484, July 1931. $\rightarrow$ pages 5

[140] L. Wu, P. A. A. F. Wouters, and F. E. Steennis. Frequency-domain transient analysis in double-circuit mixed hv overhead line-cable connection including cross-bonding. International Transactions on Electrical Energy Systems, 26(7):1408-1426, October 2015. $\rightarrow$ pages 41

[141] T.-C. Yu and J. R. Martí. A robust phase-coordinates frequency-dependent underground cable model (zcable) for the emtp. IEEE Transactions on Power Delivery, 18(1):189-194, January 2003. $\rightarrow$ pages 10 